\title{
Using Al and Machine Learning to Indicate Shale Anisotropy and Assist in Completions Design
}

\author{
Cole E. Palmer \\ cepalmer@mix.wvu.edu
}

Follow this and additional works at: https://researchrepository.wvu.edu/etd

Part of the Geological Engineering Commons, and the Other Engineering Commons

\section{Recommended Citation}

Palmer, Cole E., "Using Al and Machine Learning to Indicate Shale Anisotropy and Assist in Completions Design" (2020). Graduate Theses, Dissertations, and Problem Reports. 7870.

https://researchrepository.wvu.edu/etd/7870

This Thesis is protected by copyright and/or related rights. It has been brought to you by the The Research Repository @ WVU with permission from the rights-holder(s). You are free to use this Thesis in any way that is permitted by the copyright and related rights legislation that applies to your use. For other uses you must obtain permission from the rights-holder(s) directly, unless additional rights are indicated by a Creative Commons license in the record and/ or on the work itself. This Thesis has been accepted for inclusion in WVU Graduate Theses, Dissertations, and Problem Reports collection by an authorized administrator of The Research Repository @ WVU. For more information, please contact researchrepository@mail.wvu.edu. 


\title{
Using Al and Machine Learning to Indicate Shale Anisotropy and Assist in Completions Design
}

\author{
Cole E. Palmer
}

Thesis submitted to:

Benjamin M. Statler College of Engineering and Mineral Resources at West Virginia University In partial fulfillment of the requirements for the degree of

Master of Science in

Petroleum and Natural Gas Engineering (P.N.G.E)

Ming Gu, Ph.D., Committee Chairperson

Shahab D. Mohaghegh, Ph.D.

Samuel Ameri, M.S.

Department of Petroleum and Natural Gas Engineering

Morgantown, West Virginia

2020

Keywords: hydraulic fracturing, artificial intelligence, machine learning, fuzzy logic clustering, cmeans, completions, stage length, cluster spacing, shale anisotropy, unconventional reservoir Copyright 2020 Cole E. Palmer 


\begin{abstract}
Using Al and Machine Learning to Indicate Shale Anisotropy and Assist in Completions Design
\end{abstract}

\author{
Cole E. Palmer
}

Operating companies in the unconventional Marcellus shale play have all faced a similar and problematic issue, while attempting to produce natural gas over the last decade. Companies have quickly realized that not every perforation along their horizontal wells are producing gas. In fact, producing perforations are only ranging from $15 \%-70 \%$ of the total perforations along the horizontal wellbore [1]. This unexplained issue results in millions of dollars in lost revenue per well, in addition to the sunk cost of paying for completions that are not actually yielding any produced gas.

What is causing these perforations to have no produced gas? There are many theories being researched in the private sector and academia including: stress shadowing, proppant type and concentration, sand-outs, unconventional reservoir modeling, and improved geosteering. While any and all of those situations may have an impact on production, this study will focus on one potential issue with shale wells that may be the root cause of this phenomenon: the anisotropic nature of shale. By nature, shale is highly anisotropic, which means that the physical properties of shale change significantly from point to point in the $x, y$, and $z$ directions. This is caused by the laminar structure of the shale due to the shales formation, effecting properties in the $z$-direction, as well as widespread natural fracturing effecting properties in the $x-y$ directions [20]. Is it possible that the random and highly variable physical properties of the Marcellus shale are responsible for poor fracture propagation and production at various perforated clusters along the horizontal wellbore?

If the physical properties of the shale change considerably along the wellbore, then an area with favorable geomechanical properties for hydraulic fracturing such as Young's Modulus and Poisson's Ratio could quickly become unfavorable conditions simply a few feet away. An Artificial Intelligence and Machine Learning method called Fuzzy Logic C-Means Clustering can be used to identify these random changes in shale properties along the wellbore. This is done by gathering raw measured data from sources such as a sonic log or natural fracture log and allowing the Al algorithm to classify each half-foot of shales along the wellbore into groups of 'like' shales. These newly defined classifications of shales are grouped together to include shales with similar physical properties to each other. This can be used to identify areas of anisotropy along the wellbore that would have previously been unseen, allowing for an engineered completions design that ensures all perforated clusters will be placed against shales with similar physical properties. This is likely to result in improved overall production within a stage, since fractures would not be induced at different types or qualities of shales within a single stage. The theory is that a stage where every cluster successfully propagates a fracture will have higher production than a stage with one or two dominant fractures. The individual 
fractures may be smaller using this method, but the improved cluster efficiency could see improved production.

The use of C-Means Fuzzy Clustering is validated when clustering sonic and natural fracture log data for the MSEEL well MIP-3H, and comparing the changes in classification with a production log for the well. The changes in classification are quantified as an anisotropy indicator value (AIV). When comparing the AIV with the production, a peak and valley relationship is observed. When the AIV is high, the production is low or near zero at that given cluster. When the AIV is low, the opposite is true. In fact, the Fuzzy C-Means Clustering model was able to identify a high AIV at $88 \%$ of the non-producing clusters for MIP-3H. This suggests a strong correlation between the anisotropy of shale, and its effect on achieving a successful completions design.

The Fuzzy C-Means model can then be applied to a full horizontal wellbore sonic and natural fracture log in order to optimize a more successful completions design that is likely to see improved cluster efficiency when accounting for the shale anisotropy. 


\section{Dedications}

I would like to dedicate my thesis to my family, who has never failed to support me on my quest to achieve a higher education. To my father, thank you for always sharing your wisdom on a wide variety of topics with me. Ranging from helping in my everyday life, to supporting me through every level of education, I have depended on you many times and have never been let down. To my mother, who raised me to become the person I am today, I wouldn't be here if it wasn't for your never-ending love and support for me and your unyielding way of getting things done. Finally, to my brother, who's far reaching academic success has pushed me to do bigger and better things in my own education, I look forward to the day you get to dedicate some of your future works to me! In all seriousness, thank you all so much for helping me achieve my goals and being there for me every step pf the way. I love you all. 


\section{Acknowledgements}

First, I would like to thank Dr. Ming Gu for working so well with me over the last couple of years. I am so pleased to have conducted meaningful research that could impact the future of our industry together. I appreciate how Dr. Gu has always been there to help every step of the way, and I will never forget that he gave me the opportunity to join his research team at WVU. I would also like to thank Professor Sam Ameri, who's guidance and status as a parental figure within the college, helped bring me back to WVU to pursue my graduate degree and assisted in pairing me with Dr. Gu. Sam, your guidance and wisdom has always been cherished by me, and I will carry it with me for the rest of my life. I would also like to thank Dr. Shahab Mohaghegh and members of his LEADs study group for all of their amazing support. Finally, I want to acknowledge and appreciate the research start-up package provided by the P.N.G.E. Department at West Virginia University for funding my research, and to the Marcellus Shale Energy and Environmental Laboratory (MSEEL) for providing me a public database with which I was able to conduct and complete my research. 


\section{Table of Contents}

Contents

ABSTRACT

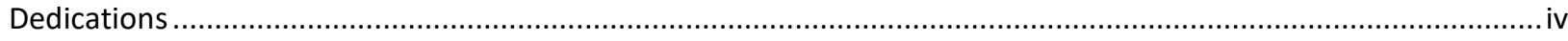

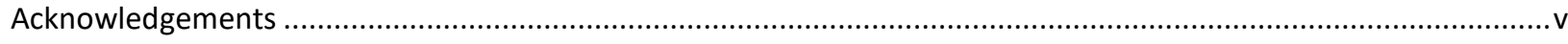

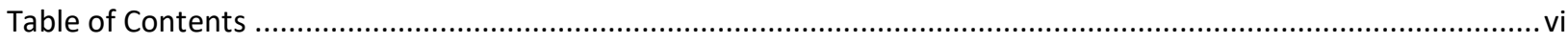

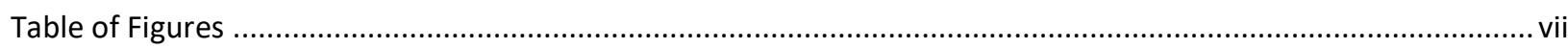

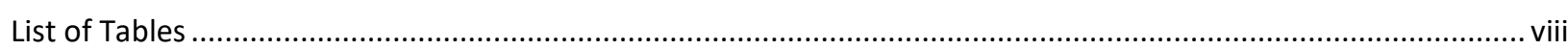

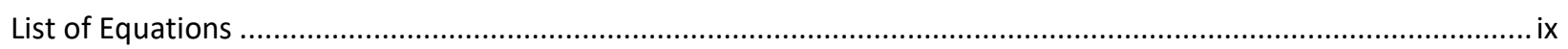

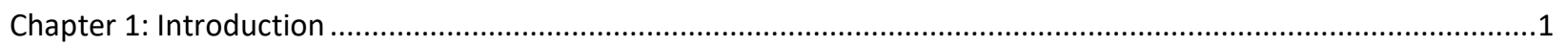

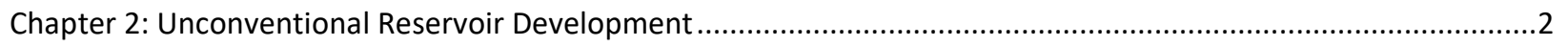

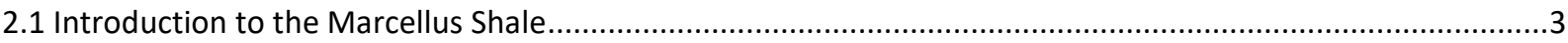

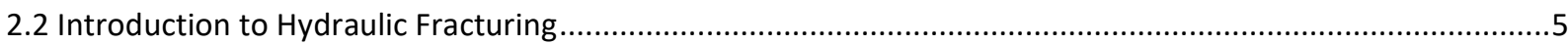

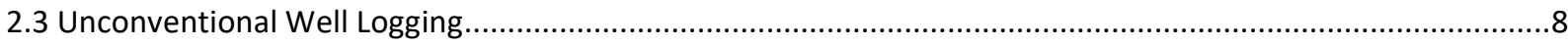

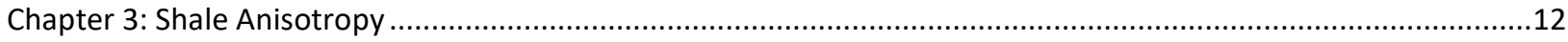

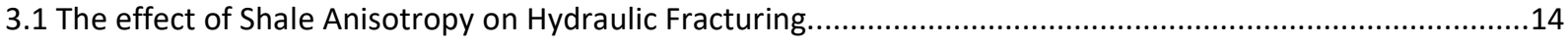

Chapter 4: Artificial Intelligence \& Fuzzy Logic C-Means Clustering ..........................................................18

4.1 Introduction to Artificial Intelligence and Machine Learning............................................................... 18

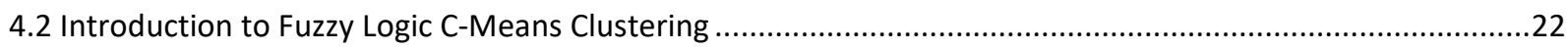

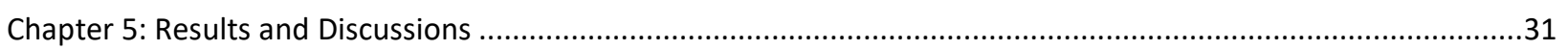

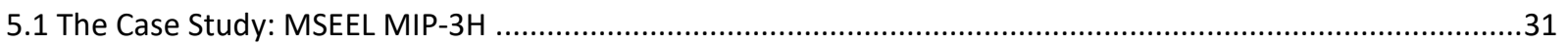

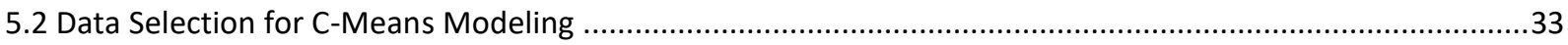

5.3 Using C-Means Fuzzy Clustering to Identify Shale Anisotropy .........................................................43

5.4 Using C-Means Fuzzy Clustering to Assist in Completions Design .......................................................52

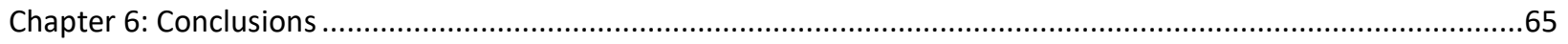

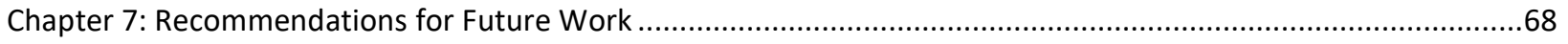

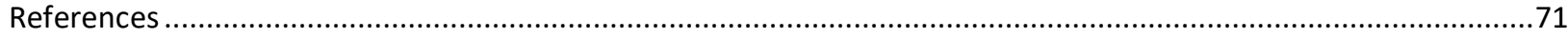

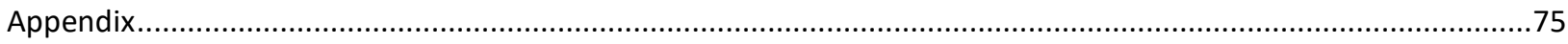

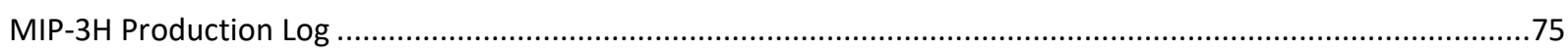

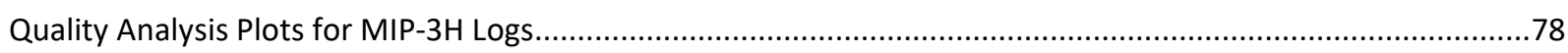

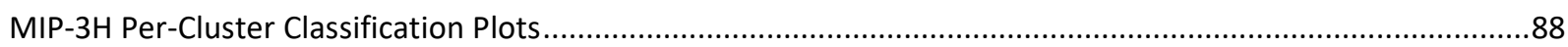

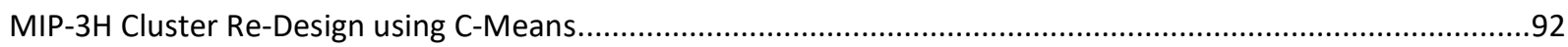

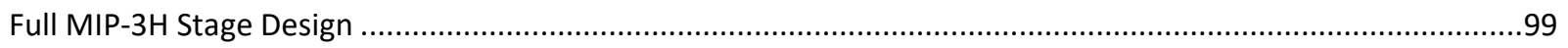

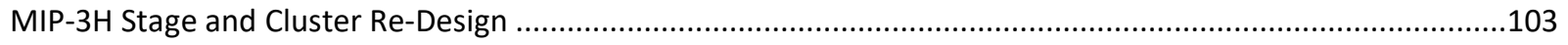




\section{Table of Figures}

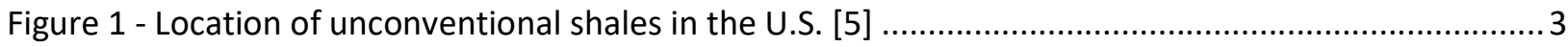

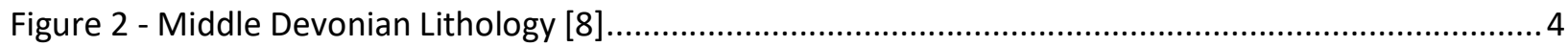

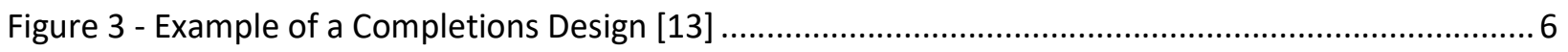

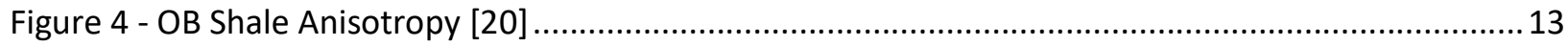

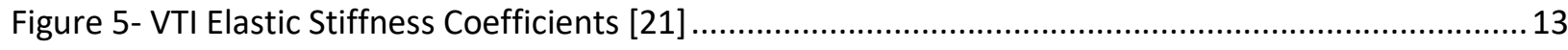

Figure 6 - Temperature Application of Fuzzy Logic [32] ................................................................. 23

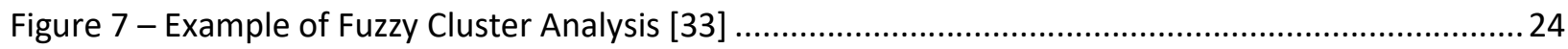

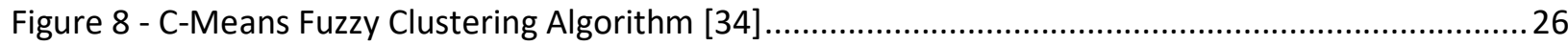

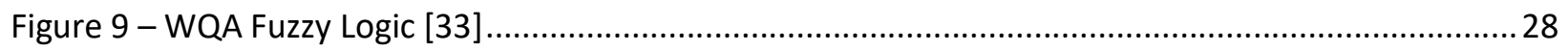

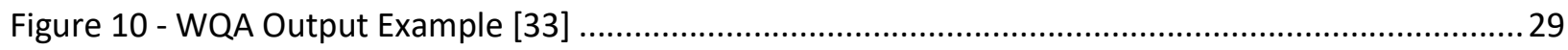

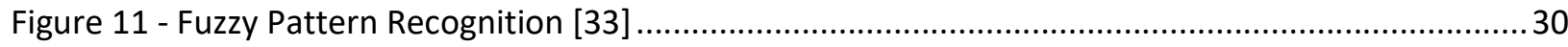

Figure 12 - Per Cluster Production Frequency Distribution .............................................................. 34

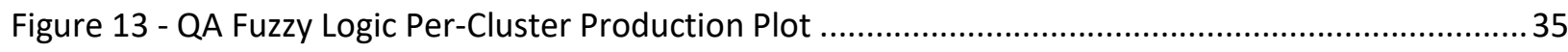

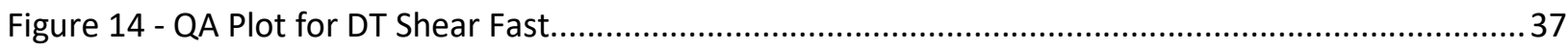

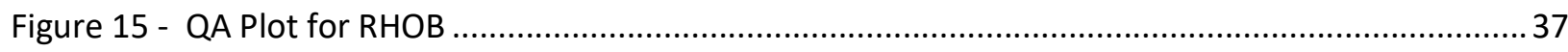

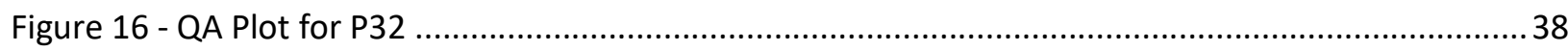

Figure 17 - QA Summary Tornado Chart (Green: high degree of influence, Yellow: manipulated data with

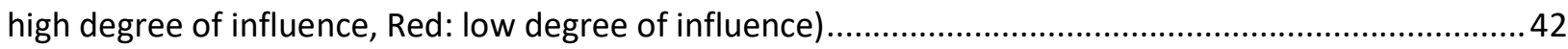

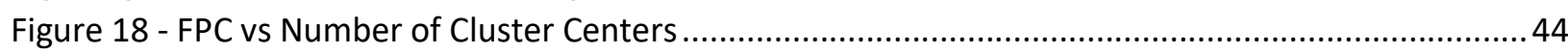

Figure 19 - C-Means Fuzzy Membership vs Production for Stage 6 ...................................................47

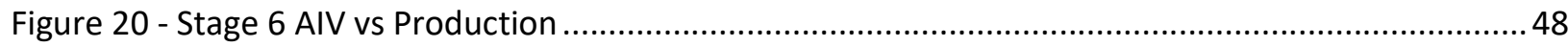

Figure 21 - C-means Fuzzy membership vs Production for Stage 13................................................ 49

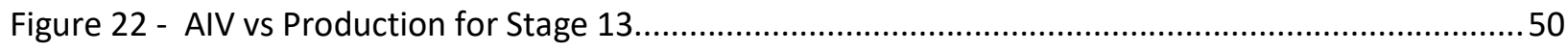

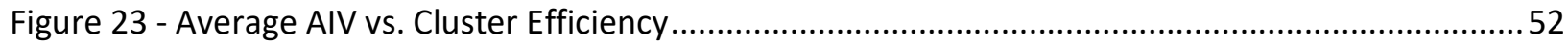

Figure 24 - FPC vs Number of Centers for Full Wellbore Clustering ....................................................53

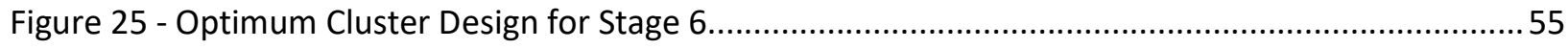

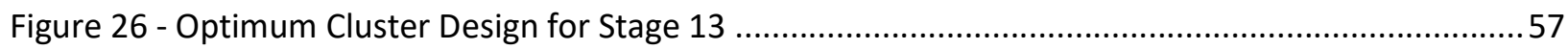

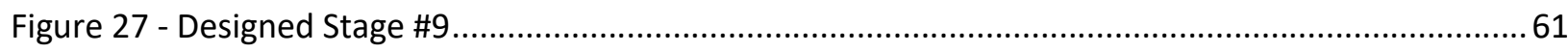

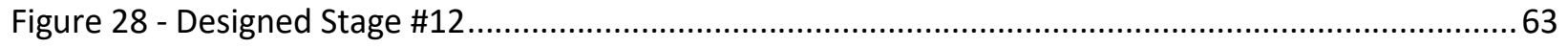




\section{List of Tables}

Table 1 - Top Unconventional Shale Plays in the U.S. (2014) [4]

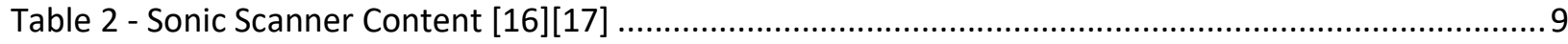

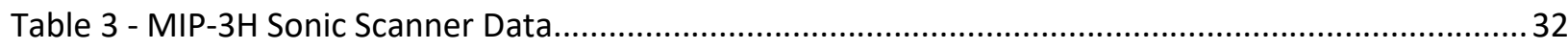

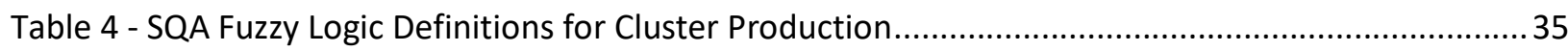

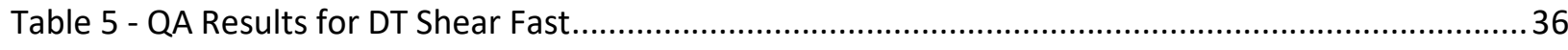

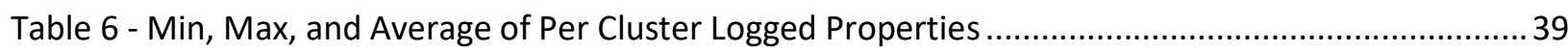

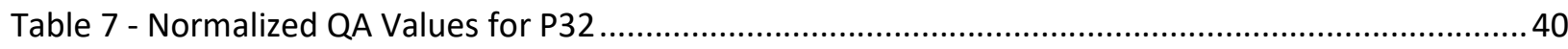

Table 8 - QA Summary (Green: high degree of influence, Yellow: manipulated data with high degree of

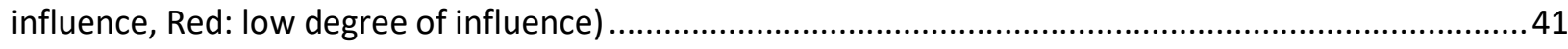

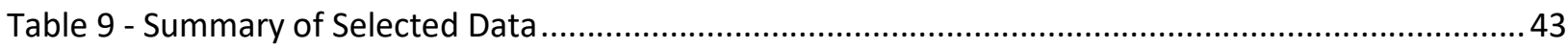

Table 10 - Breakdown of Non-producing Clusters due to Anisotropy ....................................................51 


\section{List of Equations}

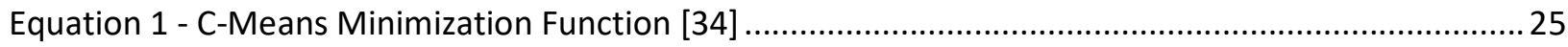

Equation 2 - C-Means Iterative Method for Cluster Optimization [34] ...............................................25

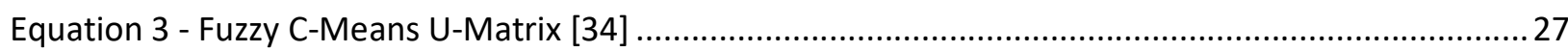

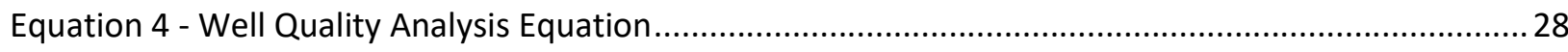

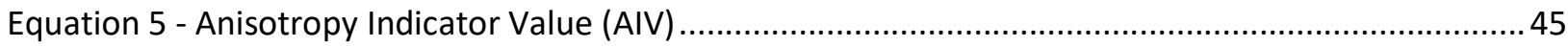




\section{Chapter 1: Introduction}

This study will demonstrate a practical use of artificial intelligence (Al) and machine learning (ML) in the form of C-Means fuzzy logic clustering, to help solve one of the most curious problems effecting unconventional shale wells: the seemingly random lack of production from a high percentage of perforated clusters for any given well.

Before the results of the study can be presented, it is important to understand the background topics that form the base of this study. Background information on unconventional shale plays, with a focus on the Marcellus shale formation, along with hydraulic fracturing and unconventional well logging will be the first topic covered. To further provide context for this study, a look at the anisotropic behavior of shales will be provided. This understanding will be key knowledge in utilizing $\mathrm{Al}$ and machine learning to identify such anisotropy along horizontal wellbores. The final set of frameworks required before describing the study and its results in a meaningful way, is defining C-Means fuzzy clustering within the realm of Al and machine learning, and how it will be applied in this project.

With the necessary background in place, the methodology and results of this project will be described in detail. This includes: the data selection process, creating a C-Means fuzzy logic algorithm to fit the needs of the project, using the C-Means algorithm on a per perforated cluster basis to compare with a production logs in order to verify the accuracy of the model, and finally to use the model to develop an optimum completions design and compare that design to the MIP-3H design. Conclusions will then be drawn from the study, and final recommendations will be made for future work in this area. 


\section{Chapter 2: Unconventional Reservoir Development}

Over the last decade in the United States, unconventional oil and gas plays have boomed around the country. This boom has been generated by advances in technology within the industry that allow shales to produce hydrocarbons cost effectively. Some of these advances include: horizontal drilling, hydraulic fracturing, carbon sequestration, and advances in drilling measurement. Unconventional shale reservoirs account for $51 \%$ of crude oil production and $67 \%$ of natural gas production in the United States according to the EIA [2]. This surge in production in the U.S. over the last decade has come amid a push to create energy independence in the United States, a dream partially realized in 2019 when the U.S. became a first-time net exporter of crude oil and all petroleum by-products [3].

As the world's known conventional reservoirs continue to become depleted, companies are turning to unconventional reservoirs to provide humanity with oil and gas for the foreseeable future. With such a large focus on expanding unconventional reservoir plays, proven reserves are continuing to be discovered throughout the country. The following figure shows the distribution of many of the largest unconventional shale plays in the United States and is accompanied by a table showing a breakdown of the top 10.

\begin{tabular}{|c|c|c|c|}
\hline Shale Play & Location & Oil Production & Gas Production \\
\hline Permian & West Texas & 1.3 million bbl/d & $5 \mathrm{bcfd}$ \\
\hline Eagle Ford & Southern Texas & $1.2 \mathrm{million} \mathrm{bbl} / \mathrm{d}$ & $6 \mathrm{bcfd}$ \\
\hline Marcellus & OH, PA, WV, NY & $50,000 \mathrm{bbl} / \mathrm{d}$ & $13 \mathrm{bcfd}$ \\
\hline Barnett & Texas & $15,500 \mathrm{bbl} / \mathrm{d}$ & $4.5 \mathrm{bcfd}$ \\
\hline Haynesville & LA, AR, TX & $57,000 \mathrm{bbl} / \mathrm{d}$ & $4.5 \mathrm{bcfd}$ \\
\hline Bakken & MT, ND & $1 \mathrm{million} \mathrm{bbl} / \mathrm{d}$ & $1 \mathrm{bcfd}$ \\
\hline Woodford & Central Oklahoma & $1,300 \mathrm{bbl} / \mathrm{d}$ & $47 \mathrm{mcfd}$ \\
\hline Granite Wash & Texas and Oklahoma & $3,200 \mathrm{bbl} / \mathrm{d}$ & $27 \mathrm{mmcfd}$ \\
\hline Utica & OH, PA, WV, NY & $1,425 \mathrm{bbl} / \mathrm{d}$ & $9.5 \mathrm{mmcfd}$ \\
\hline
\end{tabular}

Table 1 - Top Unconventional Shale Plays in the U.S. (2014) [4] 


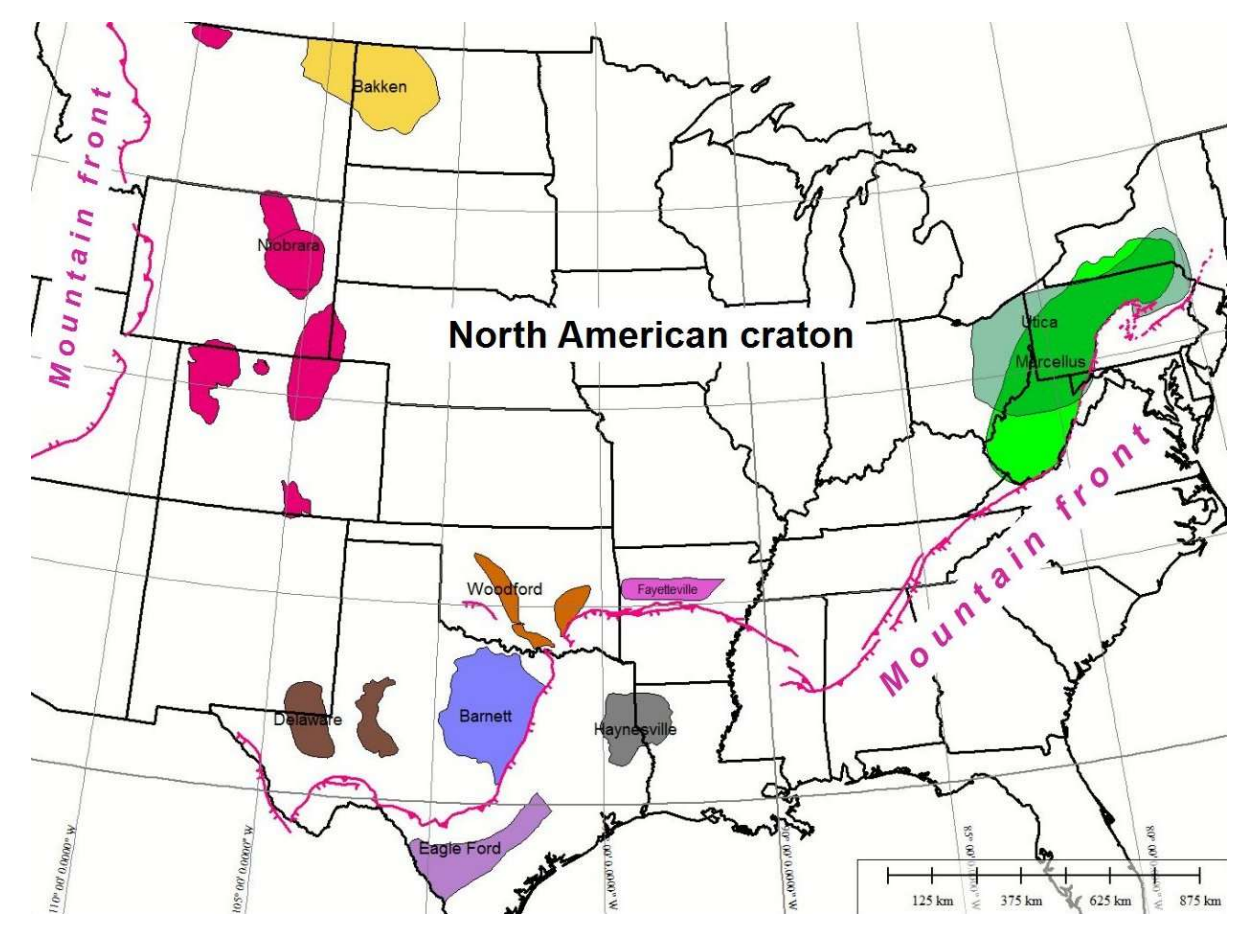

Figure 1 - Location of unconventional shales in the U.S. [5]

\subsection{Introduction to the Marcellus Shale}

The Marcellus shale, identified as light green in Figure 1, is the largest natural gas producer in the United States. At the end of 2018 the Marcellus was producing $21 \mathrm{bcfd}$ [6], with proven reserves over $77.2 \mathrm{tcf}$ [7]. The Marcellus stretches across four states, with the northernmost boundary in New York state, moving across Pennsylvania from the northeastern part of the state to the southwestern edge of the state, into eastern Ohio and northern West Virginia before the southern boundary ends at the southern border of West Virginia. The Marcellus shale region, often referred to as the Appalachian Basin, is in a position to provide the U.S. with a huge volume of gas production in the future, which has become more critical in recent times as powerplants make the switch from coal-fired to gas-fired electric generation. 
The vast nature of the Marcellus makes it geologically diverse in the manner that a well drilled in North-East Pennsylvania will see different geologic formations and properties than a well drilled in South-West Pennsylvania. Located beneath most of the Marcellus is the Utica shale (light blue in Figure 1), which is a relatively new unconventional play which has begun to be developed in more recent years. Unlike the Marcellus, which is dominated by dry gas production, the Utica produces a significant amount of crude oil at surface conditions [4].

Geologically, the Marcellus was created in the Middle Devonian time period approximately 390 million years ago. The shale itself can be described as a carbonaceous black shale, that is silty in nature. The primary mineral composition of the shales are mixed-clays, quartz, pyrite, and calcite, each with widely varying mixtures and compositions throughout the play [7]. The thickness of the Marcellus shale varies by region between $40-200 \mathrm{ft}$ in Pennsylvania, Ohio, and West Virginia. The Marcellus is located beneath other Middle Devonian formations such as: the Harrell Shale, Tully Limestone, and Mahantango Shales. The Tully limestone is typically used as a marker while drilling Marcellus Shale wells.

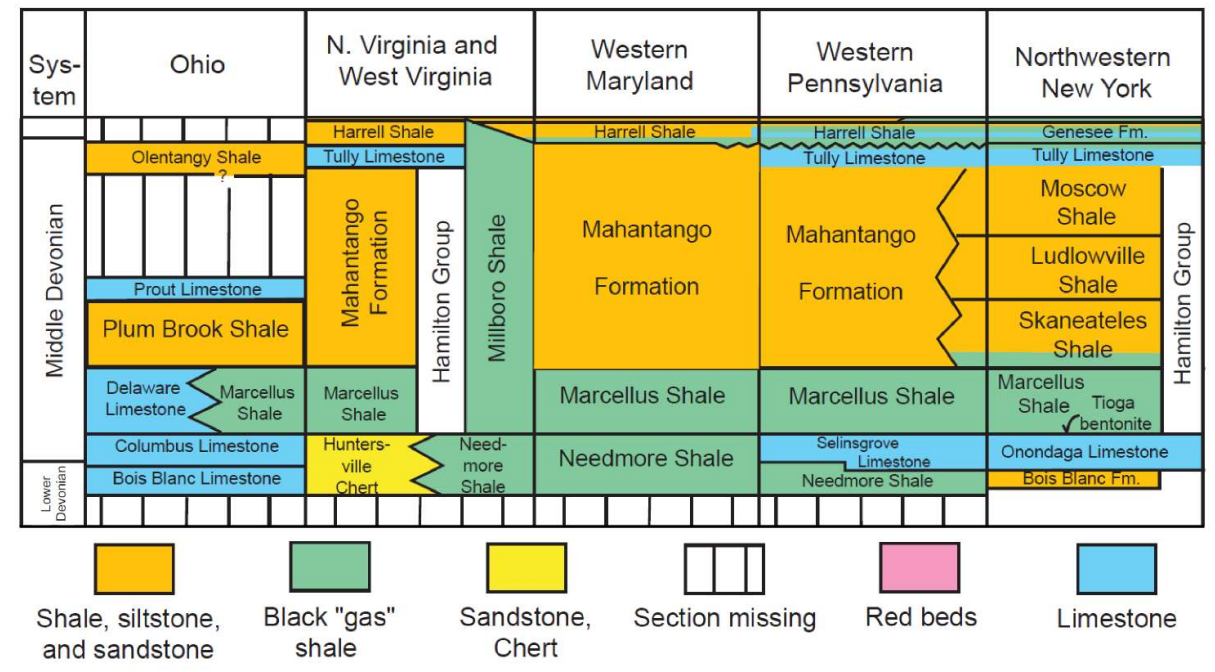

Figure 2 - Middle Devonian Lithology [8] 


\subsection{Introduction to Hydraulic Fracturing}

In order to produce hydrocarbons from unconventional shale reservoirs, two modern technologies must be implemented. Frist, a well must be drilled horizontally, with the lateral section of the well at 90 degrees kept within the pay zone of the target formation. This dramatically increase a given wells exposure to the reservoir, by having a thousand feet or more of contact with the shales. Once the horizontal well has been drilled, it must then be completed. This is done using the practice of hydraulic fracturing.

In order to fracture a well, the following procedure must be completed. A section of the horizontal well must be isolated using plugs, this isolated section of the well is referred to as the 'stage'. Stages can vary in length from 100 ' to 500 ' depending on the location, horizontal length, and the preferences of the operating company. Stages are fractured one at a time, and a well can have as many stages are necessary, again depending on the length and the design parameters selected by the operating companies' completions engineer. Within a stage, a perforating gun is run through the cased hole, and a pre-determined number of perforations are made at a pre-determined distance from each other within a stage. These perforations are referred to as 'perforated clusters' or simply 'clusters'. Operators tend to use a standard geometric completions design for stage length and perforated cluster spacing across all of their wells in a given area [11][12]. An example of a typical stage and cluster design for a Marcellus shale well would be $300^{\prime}$ stage length, with 5 clusters, each cluster 50 feet apart, with a standoff of 15 feet from the beginning and end depths of the stage [12]. 


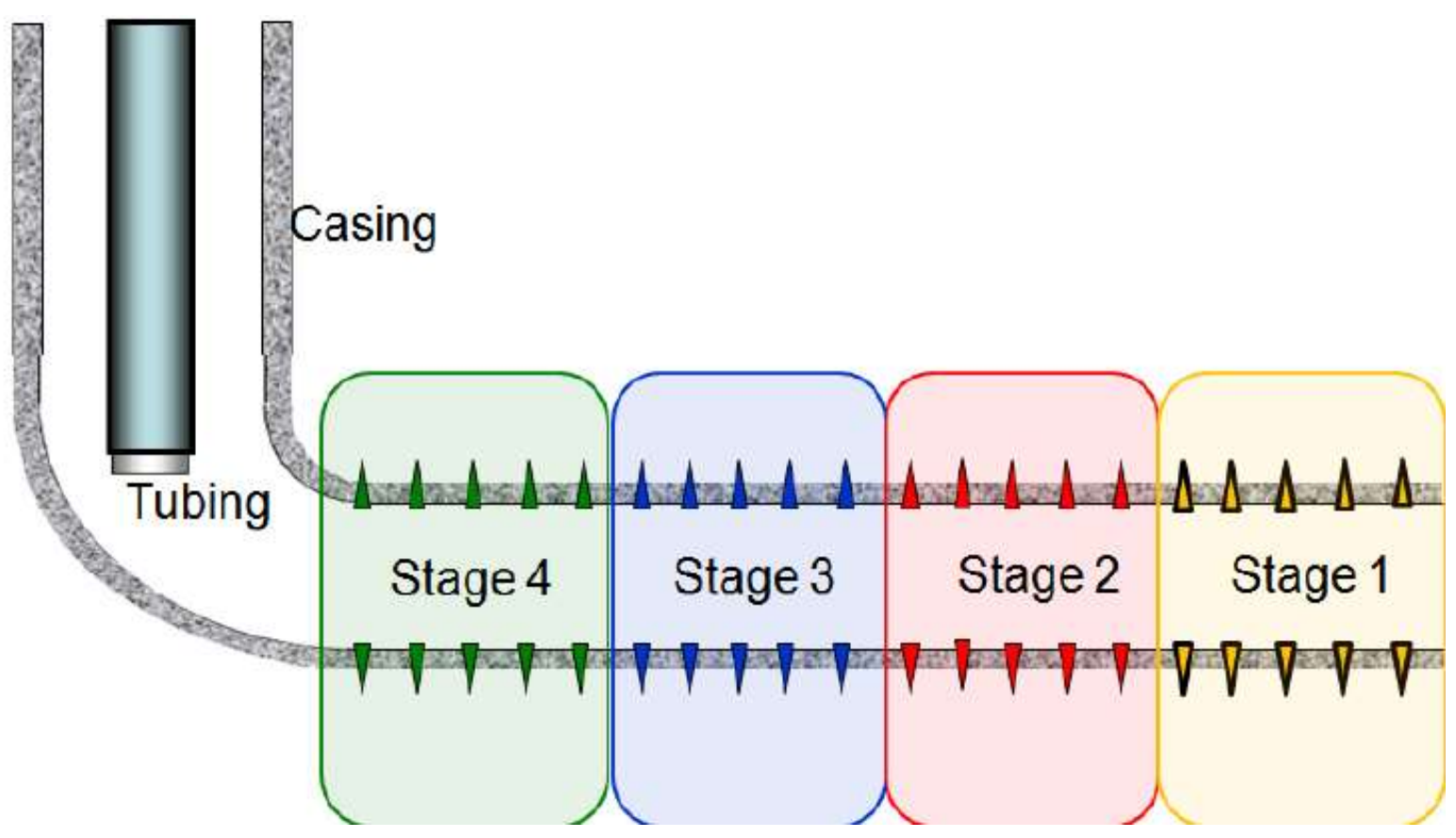

Figure 3 - Example of a Completions Design [13]

Once the stage has been perforated, the perforating gun is removed from downhole and the pumping units are attached to the wellhead via a 'frac stack'. The pumping units will then begin to pump the frac slurry downhole at extremely high pressures and rates. These pressures and rates vary by formation and the operating companies' preferences. However, a typical frac job in the Marcellus shale will pump at pressures of $8,000 \mathrm{psi}$ at a rate of $95 \mathrm{bbl} / \mathrm{min}$ of slurry [9]. The slurry used in the frac job typically contains $90 \%$ water with $9.5 \%$ sand, and $0.5 \%$ chemical additives which mainly serve to protect the downhole equipment and ensure a successful fracture treatment [10].

Once this fluid is pumped downhole and reaches the isolated stage, the water reaches its maximum pressure, which is applied to the formation via the perforated clusters. Eventually, the formation begins to break down and fracture while under the significant pressure. The sand pumped downhole in these slurries then enters these cracks as they propagate, and as the 
pressure drops the fractures begin to close onto the sand granules which hold the propagated fractures open. These steps are repeated along the entire horizontal wellbore, following the stage and perforated cluster design of the operating company.

Once the fracturing is completed, the wells contact area with the unconventional reservoir formation is upwards of 10,000 times greater than if the horizontal well attempted to produce gas without fracturing and 100,000 times greater than if a vertical attempted to produce gas without fracturing [11]. However, it's worth noting that these numbers are highly idealized and assume that every fracture propagates and extends successfully. In practice, this is not the case as only $15-70 \%$ of perforated clusters generate any propagated fractures during a fracturing treatment [1].

The causation for the inefficiency in hydraulic fracturing treatments to consistently propagate fractures and produce gas at each perforated cluster is unknown and subject to widespread studies. This study will look at shale anisotropy, the variance in the shales physical properties, as a likely cause of this issue as it relates to the geometric completions designs used throughout the industry. Other areas of study that are believed to be causing this lack of fracture propagation and gas production include: stress shadowing - where fracture propagation at one cluster is effective the geomechanical properties at another cluster, fracture hits - when propagating fractures combine to form a single network, fracture treatment variables - such as pump rates, proppant size and concentration, and geosteering issues keeping the entire lateral in the same formation. 


\subsection{Unconventional Well Logging}

In order to fully understand unconventional reservoir characteristics, properties of the formation must be measured. These measurements can be taken in many different ways, to find a wide assortment of reservoir and well development properties. Some of the common unconventional well logging techniques include: mud logging (to evaluate the status of the well, performance of drilling operations, reservoir fluids), cuttings logging (composition of fluid trapped in returned cuttings and rock mineralogy), logging while drilling or LWD (for geosteering and formation identification), wireline logging on either a cased or open hole (improved understanding of reservoir characteristics and performance), and digital slickline logging (to monitor downhole operations) [14].

Within the realm of this study, the focus will be on the open hole wireline logging method, which utilizes a sonic scanner tool and the logging while drilling (LWD) method specifically the MicroScope HD tool. The open-hole wireline logging method can provide data relating to areas of importance such as: petrophysics (formation evaluation of rock and fluid properties), geomechanics (rock stiffness and strength), geology (micro and macro geologic analysis), geophysics (large scale reservoir modeling and interpretation), and reservoir engineering (fluid flow and phase behavior in large and small scales) [15].

To analyze the geomechanical properties of an unconventional reservoir for the purpose of developing an efficient well completion design, the sonic scanner logging tool would provide the most helpful geomechanical information when trying to predict the outcome of hydraulically fracturing the unconventional formation. It is used to help identify shale 
anisotropy, favorable fracturing conditions based on a calculated young modulus and poison's

ratio, minimum horizontal stress as well as estimated pore and overburden pressures

calculated based on a gradient. All of these values can be interpreted to design a completions

program that takes into account the physical properties of the shale. An example of the values

measured by a sonic scanner tool from Schlumberger include the following with their

definitions and how these values are found:

\begin{tabular}{|c|c|c|}
\hline Logged Property: & Definition: & Calculated with: \\
\hline C11 Elastic Modulus & Elastic Stiffness Coefficient in the C11 Direction & DT Compessional, DT Shear Fast and Slow \\
\hline C12 Elastic Modulus & Elastic Stiffness Coefficient in the C12 Direction & DT Compessional, DT Shear Fast and Slow \\
\hline C13 Elastic Modulus & Elastic Stiffness Coefficient in the C13 Direction & DT Compessional, DT Shear Fast and Slow \\
\hline C33 Elastic Modulus & Elastic Stiffness Coefficient in the C33 Direction & DT Compessional, DT Shear Fast and Slow \\
\hline C44 Elastic Modulus & Elastic Stiffness Coefficient in the C44 Direction & DT Compessional, DT Shear Fast and Slow \\
\hline C55 Elastic Modulus & Elastic Stiffness Coefficient in the C55 Direction & DT Compessional, DT Shear Fast and Slow \\
\hline C66 Elastic Modulus & Elastic Stiffness Coefficient in the C66 Direction & DT Compessional, DT Shear Fast and Slow \\
\hline Borehole Deviation & Wellbore angular change from vertical (Inclination) & Direct Measurement \\
\hline DT Compressional & Measured travel time for compressional waves & Direct Measurement \\
\hline DTS Fast & Measured travel time for fast shear waves & Direct Measurement \\
\hline DTS Slow & Measured travel time for slow shear waves & Direct Measurement \\
\hline GR-Thompson & Originial measurement of gamma radition & Direct Measurement \\
\hline $\mathrm{GR}$ & Gamma Ray - Standard measurement of gamma radition & Direct Measurement \\
\hline Azi & Azimuth - Angular depature from true north & Direct Measurement \\
\hline Pore Pressure Gradient & Change in pore pressure per depth & Defined by tool operator \\
\hline Pore Pressure & Fluid pressure within the pore space of the rock & Pore Pressure Gradient \\
\hline PR Horiz & Poissons Ratio - Measure of material deformation perpendicular to applied force (Hoizontal) & DT Compessional, DT Shear Fast and Slow \\
\hline PR Vert & Poissons Ratio - Measure of material deformation perpendicular to applied force (Vetical) & DT Compessional, DT Shear Fast and Slow \\
\hline $\mathrm{RHOB}$ & Bulk Desnity - weight per given volume & Direct Measurement \\
\hline TVD & True Vertical Depth from surface reference & Borehole Deviation, Azimuth \\
\hline Iso Closure Stress Gradient & Closure stress per depth in Isotropic formations & Defined by tool operator \\
\hline Ansio Closure Stress Gradient & Closure stress per depth in Anisotropic formations & Defined by tool operator \\
\hline Iso Closure Stress & Pressure at which formation fractures close in Isotropic formations & Iso Closure Stress Gradient \\
\hline Aniso Closure Stress & Pressure at which formation fractures close in Anisotropic formations & Ansio Closure Stress Gradient \\
\hline Overburden Gradient & Overburden pressure per depth & Defined by tool operator \\
\hline Overburden Pressure & pressure resulting from the weight of overlying formations & Overburden Gradient \\
\hline YM Dynamic Hz & Young's Modulus - moving average of formation tesnise stiffness in the horizontal direction & DT Compessional, DT Shear Fast and Slow \\
\hline YM Static $\mathrm{Hz}$ & Young's Modulus - instantaneous tesnise stiffness in the horizontal direction & DT Compessional, DT Shear Fast and Slow \\
\hline YM Dynamic Vt & Young's Modulus - moving average of formation tesnise stiffness in the vertical direction & DT Compessional, DT Shear Fast and Slow \\
\hline YM Static Vt & Young's Modulus - instantaneous tesnise stiffness in the vertical direction & DT Compessional, DT Shear Fast and Slow \\
\hline
\end{tabular}

Table 2 - Sonic Scanner Content [16][17]

The contents of Table 2 come directly from the well MIP-3H from the Marcellus Shale

Energy and Environmental Laboratory (MSEEL). This project will be discussed further in Chapter

4. In addition to the geomechanical properties of shales, another important area to consider

when it comes to hydraulic fracturing design is the presence of natural fractures, and their 
intensity. These natural fractures have the tendency to open and propagate first before the formation is broken down in a hydraulic fracturing stage [18]. As a result, it would be favorable to understand these natural fractures as it relates to completion design.

Using a logging while drilling (LWD) tool such as the MicroScope HD can help achieve the goal of identifying the locations of natural fractures. The MicroScope HD takes highdefinition images of the wellbore in 360 degrees to visually detect natural fractures and their orientation. This tool has the ability to measure the resistivity to find natural fractures up to 7inches into the formation, or physically identify fractures using imaging up to 1.5 inches of formation. The tool combines using the resistivity imaging and the physical imaging using bottom nodes on the outside of the tool to create a comprehensive image detailing the presence of natural fractures. The resulting log from this tool reports the values: natural fracture count, natural fracture intensity, natural fracture dip angle, and natural fracture azimuth per half foot. The natural fracture intensity, labeled as P32, is the calculation of the total volume of open space due to the natural fractures over the half foot interval. This information provides the number of fractures in a given area and how significant the fracturing is, which are two important factors to consider when designing a fracturing treatment [19].

The tools and logs defined in this section are only the ones relevant to this project. However, the scope of well logging can be to focus on one individual well, or the relationship of reservoir properties across a specific asset or even across a specific formation. It is the backbone of asset development, since the data gathered from well logging is essential to continuously improving the project. The more data acquired, the better the understanding will be. 
However, one of the potential limitations to using conventional logging tools such as the ones described in this section for an unconventional shale play, is the depth of investigation associated with each measurement device on the logging tool. Each device will only be able to measure a small area into the formation from the wellbore, thus paining an accurate picture of near-wellbore lithology, but not necessarily what lies beyond the depth of investigation for each tool. While these tools are essential in the development of assets, it is important to bear in mind the potential error based on assuming consistency in the measurements, especially when considering the anisotropic and heterogeneous nature of the shales. 


\section{Chapter 3: Shale Anisotropy}

The definition of anisotropy states that a material's properties change along it's $x, y$, and $z$ axis. This condition of anisotropy is highly applicable to shales due to their layered structure. Shales are formed from the compaction of silt and clay particles over time and are notable in the laminar way the silt and clay 'mud' compacts. This laminar formation leads to very high levels of anisotropy in the z-direction of shales, since these laminar layers are often horizontal perpendicular to the direction of gravity [20].

However, shales are not only anisotropic in the z-direction, but also very much so in the $x-y$ direction as well. The primary cause of this is the natural fractures that occur throughout shale formations due to their very brittle nature. These can be large dimensional fractures to microfractures, each fracture contributing to shale anisotropy in the $x$-y direction. If the natural fracture offsets the laminar shale at all, the shale becomes anisotropic in the $x-y$ direction. This occurrence is similar to faulting formations, but on a much smaller scale. Figure 5 demonstrates the anisotropy in the $x, y$, and $z$ directions, where the NFs in the figure stand for natural fractures: 


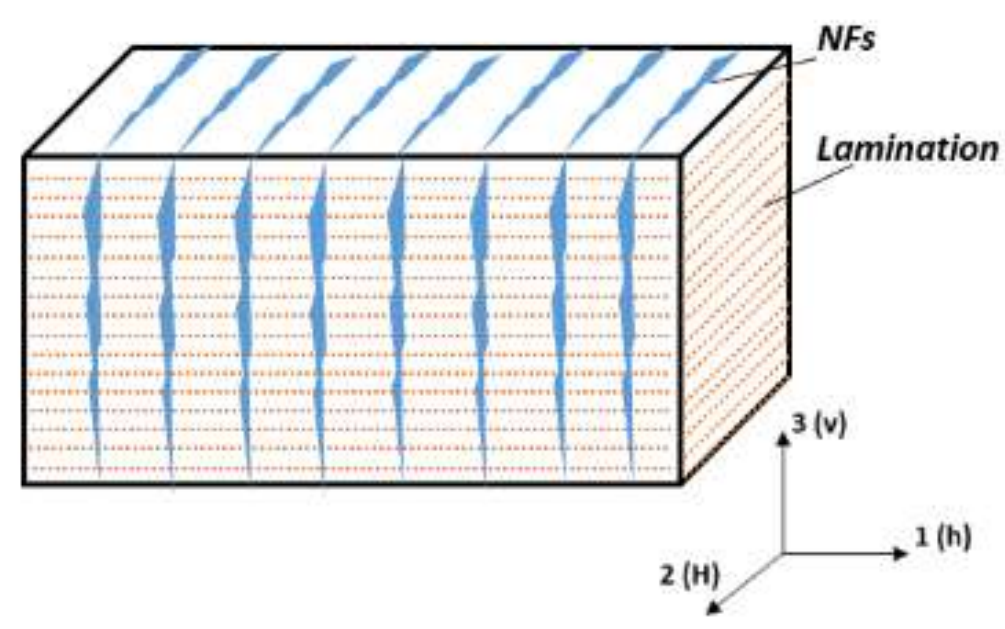

Figure 4 - OB Shale Anisotropy [20]

Shales are commonly recognized for their z-direction anisotropy due to the laminar structure of the rock. This referred to as being vertically transversely isotropic (VTI). Being a VTI material suggests that there is a vertical axis of rotational symmetry, and that the material is only anisotropic in the vertical direction and isotropic in the $x-y$ direction. This assumption allows for the use of Hooke's Law to calculate elastic stiffness coefficients. This equation is presented below:

$$
\mathrm{C}=\left[\begin{array}{llllll}
\mathrm{C}_{11} & \mathrm{C}_{12} & \mathrm{C}_{13} & 0 & 0 & 0 \\
\mathrm{C}_{12} & \mathrm{C}_{11} & \mathrm{C}_{13} & 0 & 0 & 0 \\
\mathrm{C}_{13} & \mathrm{C}_{13} & \mathrm{C}_{33} & 0 & 0 & 0 \\
0 & 0 & 0 & \mathrm{C}_{44} & 0 & 0 \\
0 & 0 & 0 & 0 & \mathrm{C}_{44} & 0 \\
0 & 0 & 0 & 0 & 0 & \mathrm{C}_{66}
\end{array} \mid\right.
$$

Figure 5- VTI Elastic Stiffness Coefficients [21]

These elastic stiffness coefficients are the same ones reported by Schlumberger's sonic scanner log defined in Chapter 1. As a result, the stiffness coefficients calculated by the Sonic 
Scanner tool and the Young's Modulus and Poisson's Ratio's derived from these values are only accounting for anisotropy in the z-direction. However, it is known that shales are not only anisotropic in the z-direction, but also in the $x-y$ direction as well. Being anisotropic in the $x-y$ direction is referred to as horizontally transversely isotropic ( $\mathrm{HTI})$. This means that on horizontal axis of rotational symmetry, the material is considered isotropic. Since shale is anisotropic in all three axes of symmetry, it is neither VTI nor HTI. In reality, it is orthorhombic (OB) [20].

This suggests that the industry-wide use of a VTI assumption for shales to calculate its geomechanical properties are likely inaccurate [20]. As of now there are no well-established acoustic log interpretation algorithms to calculate the elastic stiffness coefficients, and therefore Young's Modulus and Poisson's Ratio, for OB shales

\subsection{The effect of Shale Anisotropy on Hydraulic Fracturing}

One of the effects of shales being formed in a laminar fashion, due to the clay minerals tending to be deposited in a parallel predisposition, is the resulting brittleness of the shales. The high brittleness of shales contributes to the extensive growth of an induced fracture network following hydraulic fracturing operations. However, the brittleness of the shale along with the shales elastic properties, very significantly throughout the formation because of shale anisotropy [21].

Being able to identify areas of optimum reservoir rock in terms of elastic properties, brittleness, and strength values along a horizontal wellbore could be critical to improving unconventional well production. By identifying these optimum areas to fracture the reservoir rock, the fracture growth could be significantly increased. Currently, a Brittleness Index (BI) 
derived from elastic stiffness coefficients by making the shale VTI assumption is the best way to evaluate the best fracture locations for a shale well [21]. The primary issue with using many of the current BI models such as the one proposed by Salah [21] to evaluate a shale well is that it is based on the VTI anisotropic assumption, where in reality the shale has OB anisotropy.

Computer simulation has been done to demonstrate how the anisotropy of shales has a significant impact of hydraulic fracture propagation [22]. Likewise, laboratory experiments conducted on core samples from Marcellus shale wells support the notion that shale anisotropy highly impacts shale properties such as induced fracture conductivity, closure stress, Young's Modulus, and Poison's Ratio. The core samples in this experiment resulted in different fracture propagation and fracture conductivity when taken from the same well. This difference was determined to be caused by the anisotropic behavior of the shales strength properties [23].

Computer modeling and core samples tested in the lab confirm that shales anisotropy has a significant impact on how induced fractures preform. However, what is uncertain is how the shale anisotropy can be measured and identified to then be used to design well completions. Using the VTI assumption for shales is inadequate, as are derived values including proposed Brittleness and Anisotropy Indexes. Other methods include using a derived acoustic anisotropy log measured from a sonic logging tool. The results of these anisotropy logs are considered difficult to understand and interpret due to issues such as: the borehole deviation, wellbore condition, proper tool centralization, inaccurate sonic source and receiver matching and ability for the tool to directly detect anisotropy [24]. Likewise, tools directly measuring anisotropy are more effective measuring a vertical wellbore [25]. While there are multiple logging tools that directly measure anisotropy available to use on horizontal wells, there is not a 
clear way to accurately interpret such measurements using conventional means. Using modern tools such as artificial intelligence and machine learning can open new doors when it comes to selecting the most representative data.

Using VTI based parameters or an anisotropy values directly measured from a sonic logging tool could be potentially led to issues such as underestimating formation stresses and breakdown pressures [26]. This could to being as inaccurate as simply using an isotropic approach. Assuming formation isotropy, or incorrectly assuming VTI, can lead to overestimation of fracture width which can have a negative impact on the completions based on the proppant design. This leaves significant amounts of uncertainty when planning hydraulic fracturing or drilling programs. It is critical to properly understand and classify formation heterogeneity and anisotropy when deciding where to land a horizontal wellbore, where to place perforated clusters and how long to design fracture stages [27].

A study conducted by Hadi et al focusing on the effects of fracturing rock with homogeneous vs heterogeneous geomechanical properties has demonstrated that the homogenous nature of the rock is a more critical factor in a successful hydraulic fracture than geomechanical properties themselves [28]. Shale is by nature anisotropic, so it would be incorrect to consider it isotropic along certain areas of the wellbore. However, you can consider certain lengths of shales along the wellbore to be homogenous, and therefore have like properties, while still being anisotropic rock. Within a certain stage length, it should possible to find areas of homogeneity to hydraulically fracture. It may be advantageous to design fracture stages in order to fracture areas of 'like' formation based on their raw un-manipulated data 
from geomechanical logging tools, instead of using values calculated based on an isotropic or VTI assumption such as Young's Modulus and Poisson's Ratio as is common in industry. 


\section{Chapter 4: Artificial Intelligence \& Fuzzy Logic C-Means Clustering}

Advancement in technology has led to the widespread implementation of artificial intelligence (AI) and machine learning $(\mathrm{ML})$ across the world. With applications ranging from facial recognition on a cellphone to managing 'big data' in order to create user-customized advertisements on social media, the presence of $\mathrm{Al}$ and $\mathrm{ML}$ continues to expand every single day. With this seemingly exponential growth, and $\mathrm{Al}$ and $\mathrm{ML}$ has begun branching out into many different industries outside of Silicon Valley. From analytical uses in medicine and accounting, to predictive uses in gambling, Al and ML has proven its use beyond 'Big Tech' [29]. Al and ML has drawn considerable interest from the oil and gas industry, to help predict and explain many of the unknowns surrounding unconventional reservoirs.

\subsection{Introduction to Artificial Intelligence and Machine Learning}

Historians consider Alan Turing, a British mathematician, computer scientist, statistician, philosopher, cryptanalyst and theoretical biologist to be the father of modern-day computing and Al. His work in the 1940s and 50s led the way for the future development of Al and ML by creating the idea that a computer could mimic the thinking of a human brain. Work on creating a method of computation that can mimic the human brain continued after Turing, by neuroscientist Warren McCulloch and logistician Walter Pitts [30]. By drawing influence from different areas of study such as philosophy, computer science, cognitive science, mathematics, and physiology, scientists continued to develop this emerging technology. The official birth of Al can be traced back to a project completed at Dartmouth University in 1956, which was focused on problem solving with symbolic methods [31]. 
The United States Department of Defense (DoD) took interest in this technology and continued the work on using computers to recreate human logic. The Defense Advanced Researched Projects Agency (DARPA) began implementing Al technologies as early as the 1970s by instituting street mapping projects for the DoD and even created digital personal assistants as early as 2003. These achievements came long before Al and ML boomed in the public sector via Silicon Valley giants such as Google, Microsoft, Apple, and Facebook [31].

The lag behind in the public sector use of AI and ML can be attributed to early use of the term "Artificial Intelligence" referring to a rule-based system of decision making based on Aristotelian logic. Significant capital investment in such technology resulted in little to know positive contributions. This led to a widespread denunciation of Al as it existed, with many referring to the idea as a hoax. However, this failed system relied on ruled based decisions. True Al and Machine learning as we know it today relies entirely on data. This is known as datadriven artificial intelligence [30].

Today's artificial intelligence can be defined as a method of mimicking the thinking of the human brain in order to solve complex problems, while machine learning is a subset of artificial intelligence that trains machines how to learn [30]. There are three primary types of Al and ML in common use today: artificial neural networks, fuzzy set theory, and evolutionary optimization. Each method recreates a function of the human brain or human biology in their own way. Artificial neural networks function by mimicking the way neurons function in the human brain to learn via using data to train algorithms to identify patterns and make predictions. Similarly, fuzzy set theory mimics human logic in order to learn and avoid a twovalue yes or no decision, instead using logic to create a decision on a spectrum. Finally, 
evolutionary optimization imitates Darwin's theory of evolution in order to implement natural selection to optimize complex problems [30].

The human brain performs many incredible functions with speed and efficiency that is unmatched by today's technologies. The process our brain's neurons use to learn that is mimicked by $\mathrm{Al}$ and $\mathrm{ML}$ is: observation, data collection, trial and error, pattern recognition, and practice to continue improvement [30]. An example of this is learning to walk on ice.

Observation shows the ice is slippery. Trying to step on it quickly results in a fall. That is a data point. Try again with a trial and error method. A trend emerges that walking slowly on ice does not result in a fall. Continuing to walk on ice makes it less and less likely a fall will occur due to improvements in walking methods.

This is an example of data-driven learning, and how the human brain isn't forced into a 'walk fast on ice' or 'walk slow on ice' solution. Al and Machine Learning can be further categorized into two prominent categories: supervised and unsupervised learning. Supervised learning requires a large amount of data, but also user influence to attain the desired results. Because of this, the user must have extensive knowledge, or domain expertise, in both the domain for which $\mathrm{Al}$ and $\mathrm{ML}$ is being utilized to solve a problem as well as the Al and ML itself. An example of supervised $\mathrm{Al}$ and $\mathrm{ML}$ in the real world are emails being sorted into a spam folder. The action of sorting email requires 'supervision' in the manner that the $\mathrm{Al}$ and $\mathrm{ML}$ algorithm must know what kind of emails to sort. It is not able to make that determination on its own. Alternatively, unsupervised Al and machine learning does not be instructed to make decisions. Unsupervised $\mathrm{Al}$ and $\mathrm{ML}$ algorithms are designed to discover patterns in data on their 
own. Domain expertise is still required to interpret the results of unsupervised $\mathrm{Al}$ and $\mathrm{ML}$ algorithms [30].

$\mathrm{Al}$ and $\mathrm{ML}$ are able to find solutions to problems without using the traditional engineering approach of performing numerical operations to calculate solutions. Sometimes, there are problems too difficult to calculate with an equation, which leads to assumptions and simplifications of the equations, leading to uncertainty in calculated results. Al and Ml algorithms rely exclusively on data, in the form of data analytics. Data analytics follows an inductive reasoning path by moving from a big picture to a specific result. This varies from statistics which uses deductive reasoning to study a specific occurrence and then generalize based on the results. Likewise, statistics uses pre-defined equations and models to interpret a set of data, whereas data analytics uses the data to build a model and inductively finds a patten in it. Data analytics has the ability to interpret data by finding patterns that had previously not been seen and use these patterns to predict enabling better decision making in the future [30].

Data analytics with $\mathrm{Al}$ and $\mathrm{ML}$ algorithms have been proven to be effective at modeling physics and solving complex problems. However, when these algorithms are modified with equations and manipulated data, they become referred to as hybrid-modeling. This is modeling that uses $\mathrm{Al}$ and ML algorithms and pre-defined equitation's to derive an analytical; or predictive solution. This hybrid-modeling is an ineffective approach to data analytics and can attributed to the lack of understanding of $\mathrm{Al}$ and Machine learning in its users. It is extremely important to recognize that in order to preform accurate data analytics, in place of traditional physics-based problem solving, that the Al and ML algorithm be entirely data-driven [30]. Allowing the $\mathrm{Al}$ and $\mathrm{ML}$ algorithm to find the patterns in data, whether supervised or 
unsupervised, is what allows them to work effectively and what differentiates the Al of today with the 'Al' methods in the late $20^{\text {th }}$ century.

\subsection{Introduction to Fuzzy Logic C-Means Clustering}

To understand the fuzzy logic C-Means clustering methodology, one must first understand it's theoretical origins. This Al algorithm is based on the fuzzy set theory, which states that items can be described to a degree, instead of being described precisely. This is a common function of the human brain, to think of things on spectrum instead of simply black and white. Fuzzy set theory is opposed to the conventional set theory, the idea that an object can be defined as either a member of a class, or not a member of a class [30]. There is no in between. A real-world example of the human brain utilizing fuzzy set theory is by categorizing a person as old. Is 70 years old, but 69 years 364 days young? The brain thinks of age as a spectrum, where a 70-year-old is considered to belong to the class 'old' more than someone who is 60 but less than someone who is 80 . There is no clear definition between old and young. The following figure demonstrates the same concept for cold, warm and hot days. Where 60 degrees Fahrenheit does not belong to only one of those categories, but partially to being cold and partially to be warm with a slightly higher membership to the cold classification: 


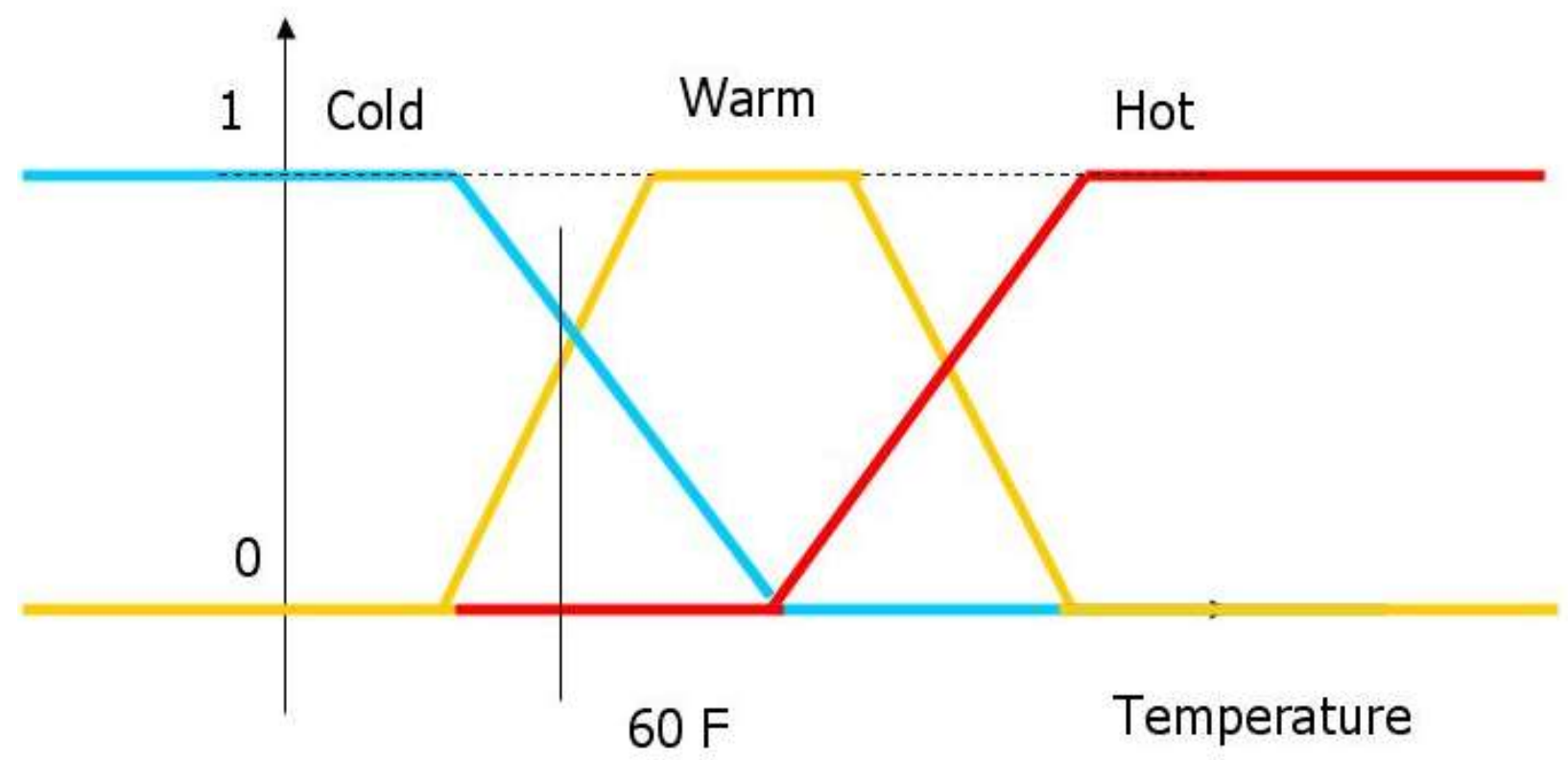

Figure 6 - Temperature Application of Fuzzy Logic [32]

Fuzzy set theory acts as a way to quantify this using a range of class membership, calculated with mathematics to simulate human thinking. It allows for a degree of 'vagueness' when describing an object's characteristics. Fuzzy set theory was utilized in creating fuzzy logic, which is the mathematical method used to implement fuzzy set theory in Al and ML. Fuzzy logic mimics human reasoning by classifying inputs as partial members to a class, using approximate information, and perception-based computation [30]. An example of training machines to use fuzzy logic to think like humans is implementing automatic braking systems in cars. The human brain doesn't think about exactly how many feet away from a red light do you need to press the break. It is done with approximate thinking and is being replicated in vehicles.

Fuzzy clustering is a numerical method that utilizes fuzzy logic to classify similar sets of data. Traditional statistical clustering methods, including K-Means clustering, will place data solely into a defined class such as 0,1 , or 2 . Fuzzy cluster analysis will place each data point as a 
partial member of each defined class [33]. Figure 7 demonstrates how fuzzy cluster analysis works on a 2-dimensional set of data. In this example the number of cluster centers is defined as two, and in practice the locations of the cluster centers will be found with the Al algorithm. The distance of point from these centers is what defines their membership.

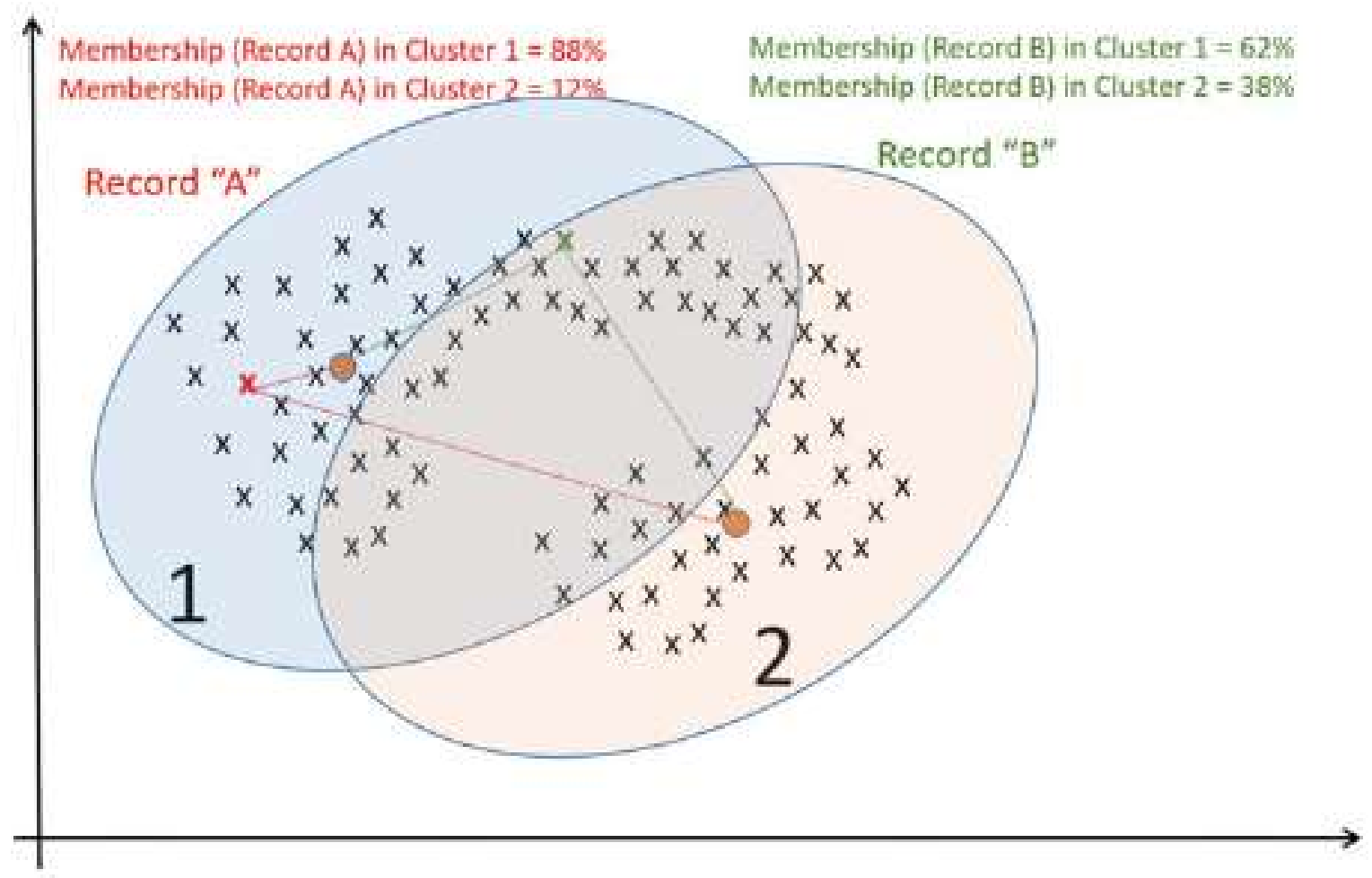

Figure 7-Example of Fuzzy Cluster Analysis [33]

Fuzzy C-Means clustering is one of the most common fuzzy clustering algorithms in use today. Originally developed by Dunn and Bezdek through their theoretical work in 1973 and 1981 respectively, Fuzzy C-Means is now being implemented as an Al and ML algorithm used in pattern recognition [34]. Equation 1 will demonstrate the minimization function that the CMeans fuzzy clustering algorithm is built on. The variables used in this equation and their definitions are: " $m$ is any real number greater than $1, u_{i j}$ is the degree of membership of $x_{i}$ in 
the cluster $j, x_{i}$ is the ith of $d$-dimensional measured data, $c_{j}$ is the d-dimension center of the cluster, and $\mid\left\|^{*}\right\|$ is any norm expressing the similarity between any measured data and the center" [34].

$$
\begin{gathered}
J_{m}=\sum_{i=1}^{N} \sum_{j=1}^{C} u_{i j}^{m}\left\|x_{i}-c_{j}\right\|^{2} \quad, \quad 1 \leq m<\infty \\
\text { Equation 1 - C-Means Minimization Function [34] }
\end{gathered}
$$

When the fuzzy logic partitioning is being carried out by the Al and $\mathrm{ML}$ algorithm, it is done using an iterative method of the equation presented in Equation 1. Every pass through the iteration updates both the fuzzy membership value $\left(u_{i j}\right)$ and the location of the cluster centers $\left(c_{j}\right)$ [34]. The equations used in the optimization of the objective function presented in Equation 1 are shown in Equation 2 below:

$$
u_{i j}=\frac{1}{\sum_{k=1}^{C}\left(\frac{\left\|x_{i}-c_{j}\right\|}{\left\|x_{i}-c_{k}\right\|}\right)^{\frac{2}{m-1}}}, \quad c_{j}=\frac{\sum_{i=1}^{N} u_{i j}^{m} \cdot x_{i}}{\sum_{i=1}^{N} u_{i j}^{m}}
$$

This iteration is stopped when the following conditions are met: "when $\max _{i j}\left\{\left|u_{i j}^{(k+1)}-u_{i j}^{(k)}\right|\right\}<\epsilon$, where $\varepsilon$ is a termination criterion between 0 and 1 , and $k$ are the iteration steps" [34]. Ultimately this procedure will converge to the local minimum $J_{m}$ (also 
known as a saddle point) [34]. The entire mathematical operation behind the scenes of the CMeans fuzzy clustering algorithm can be summarized by the following steps:

1. Initialize $U=\left[u_{i j}\right]$ matrix, $U^{(0)}$

2. At $k$-step: calculate the centers vectors $C^{(k)}=\left[c_{j}\right]$ with $U^{(k)}$

$$
c_{j}=\frac{\sum_{i=1}^{N} u_{i j}^{m} \cdot x_{i}}{\sum_{i=1}^{N} u_{i j}^{m}}
$$

3. Update $U^{(k)}, U^{(k+1)}$

$$
u_{i j}=\frac{1}{\sum_{k=1}^{c}\left(\frac{\left\|x_{i}-c_{j}\right\|}{\left\|x_{i}-c_{k}\right\|}\right)^{\frac{2}{m-1}}}
$$

4. If $\left\|U^{(k+1)}-U^{(k)}\right\|<\epsilon$ then STOP; otherwise return to step 2.

Figure 8 - C-Means Fuzzy Clustering Algorithm [34]

The result of running this algorithm is each datapoint having a degree of membership to each cluster based on the location of the cluster centroid. The output of the algorithm is in the function of the U-Matrix, shown in Equation 3 (b) for a 2-cluster example. Equation 3 (a) shows the output of a K-Means hard clustering algorithm, where each data point is a full member to either class 0 or class 1 (in a 2-cluster example). The C-Means algorithm can also output the location of the cluster centroids for the selected number of clusters at the users discretion. 


$$
U_{M W C}=\left[\begin{array}{cc}
1 & 0 \\
0 & 1 \\
1 & 0 \\
. . & . . \\
0 & 1
\end{array}\right] \quad U_{M K C}=\left[\begin{array}{cc}
0.8 & 0.2 \\
0.3 & 0.7 \\
0.6 & 0.4 \\
. . & . . \\
0.9 & 0.1
\end{array}\right]
$$

Equation 3 - Fuzzy C-Means U-Matrix [34]

By default, C-Means fuzzy clustering is an unsupervised method of Al and ML. However, utilization of C-Means as a supervised $\mathrm{Ai}$ and $\mathrm{ML}$ algorithm can be accomplished by applying domain expertise to the data being analyzed. In the Oil and Gas Industry, petroleum engineers are properly suited to interpret geological, well logging, and production data that would be suitable to be tested with C-Means fuzzy clustering [30]. By applying domain expertise to such an analysis, data can be preprocessed and selected with an understanding that the data passed to the algorithm will provide the most accurate and optimum results to a desired study.

One such way to select data for a supervised C-Means fuzzy clustering algorithm was developed by Dr. Shahab Mohaghegh, which is called Well Quality Analysis (WQA). WQA is conducted by first assigning wells to 'poor', 'average' or 'good' classes based on their production. This assignment is done using a fuzzy logic definition, where some well production values may not simply belong to only 'poor' or 'average' but fall somewhere in between and therefore belong to both classifications. The assignment of 'poor', 'average' or 'good' well production can be decided by the user based on the production distribution for a set of wells [33]. Figure 9 shows an example of a well with 117,565 MMCF after 180 days of production has a membership value of 0.77 for the 'poor' class and 0.23 to the 'average' class. 


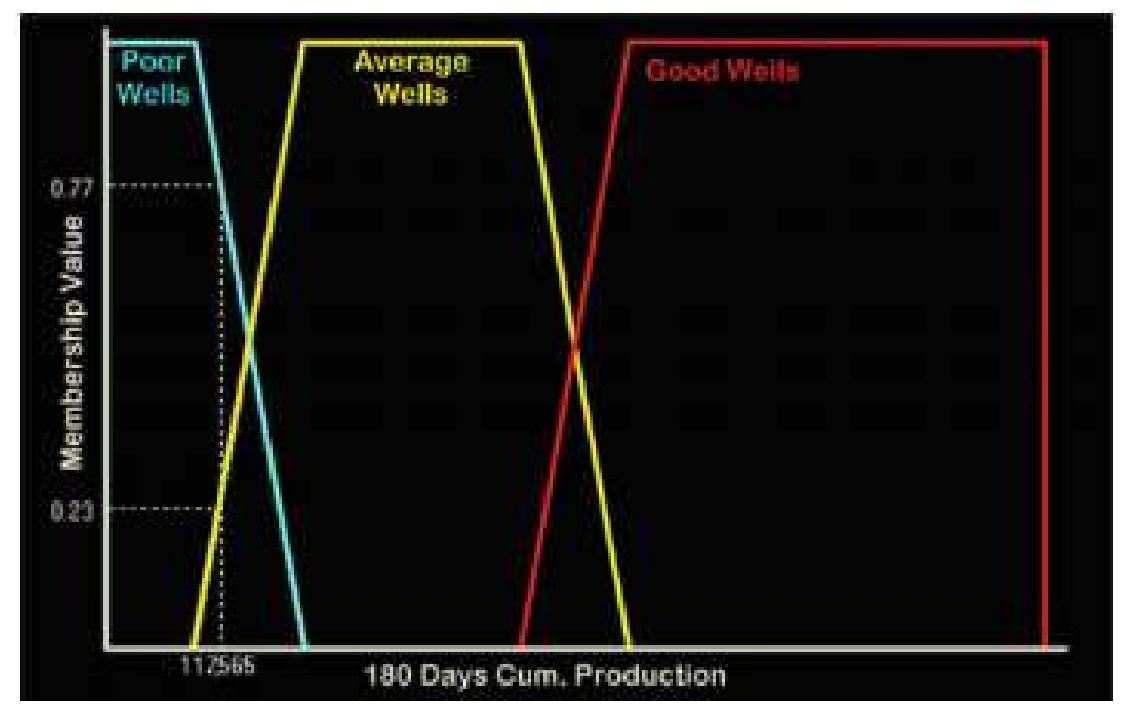

Figure 9-WQA Fuzzy Logic [33]

This fuzzy membership for each well can be applied to a specific parameter being analyzed to see the impact of parameters on on the wells production. For example, number of stages for the well shown in Figure 9 would be multiplied by the membership value of 'poor', 'average' or 'good' to result with a number of stages relative to each membership value. For a dataset with many wells, the cumulative relative stages for each class of 'poor', 'average' or 'good' can be summed and divided by the membership function totals for all of the wells to show the average number of stages for the 'poor', 'average' or 'good' wells as summarized in Equation 4, where $x$ represents the parameter in question and $\mu$ represents the membership function for the well $i$. [33]. This equation is calculated for the 'poor', 'average' or 'good' memberships.

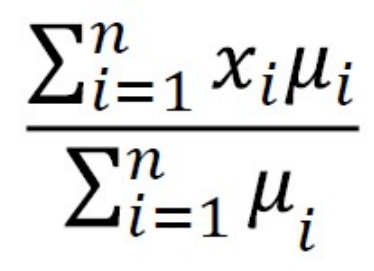


An example WQA output plot is shown in Figure 10, which demonstrates the average number of stages for each class membership. [33]:

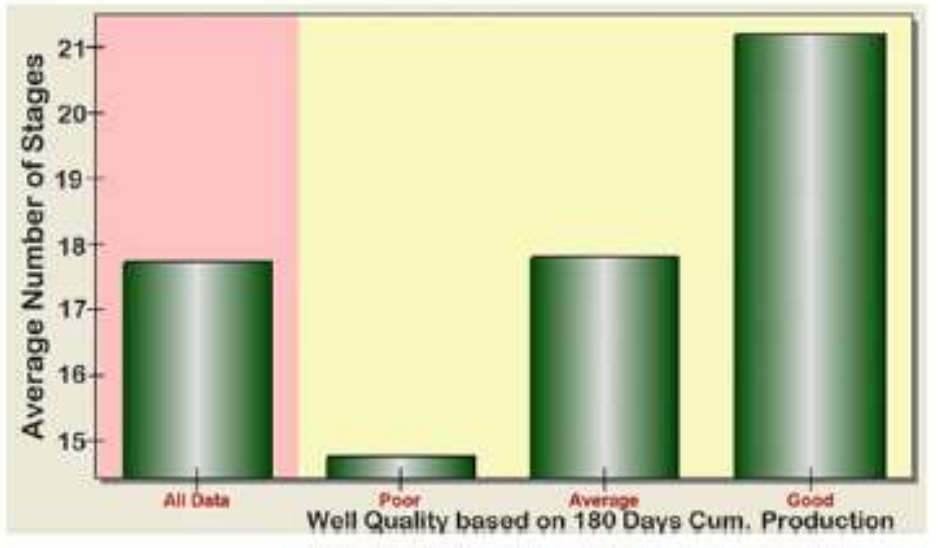

Figure 10 - WQA Output Example [33]

Figure 10 shows that there is a direct impact on the number of stages vs the quality of a wells production. The trend dictates the more stages in a well, the better the well will perform when producing gas. WQA is useful to identify seemingly unknown or hidden trends in data to determine what parameters can be seen to have a significant impact, either positive or negative, on production [33]. This can be used to select data for future C-Means clustering based on their impact on production (or any other tested variable.) WQA can be taken a step further and applied to a concept called Fuzzy Pattern Recognition. This concept follows the same concept as WQA but is used to optimizes the number of clusters applied to the well production. While discussing WQA, an example with three classes ('poor', 'average' or 'good') was used. However, there can be as many or little classes as necessary. Fuzzy Pattern recognition figures out the optimum number of classes in order to apply a much greater granularity. Instead of presenting the trends as a bar chart, there can be a large number of 
classes to the point where the results can be presented as a continuous line as demonstrated in

Figure 11 [33].

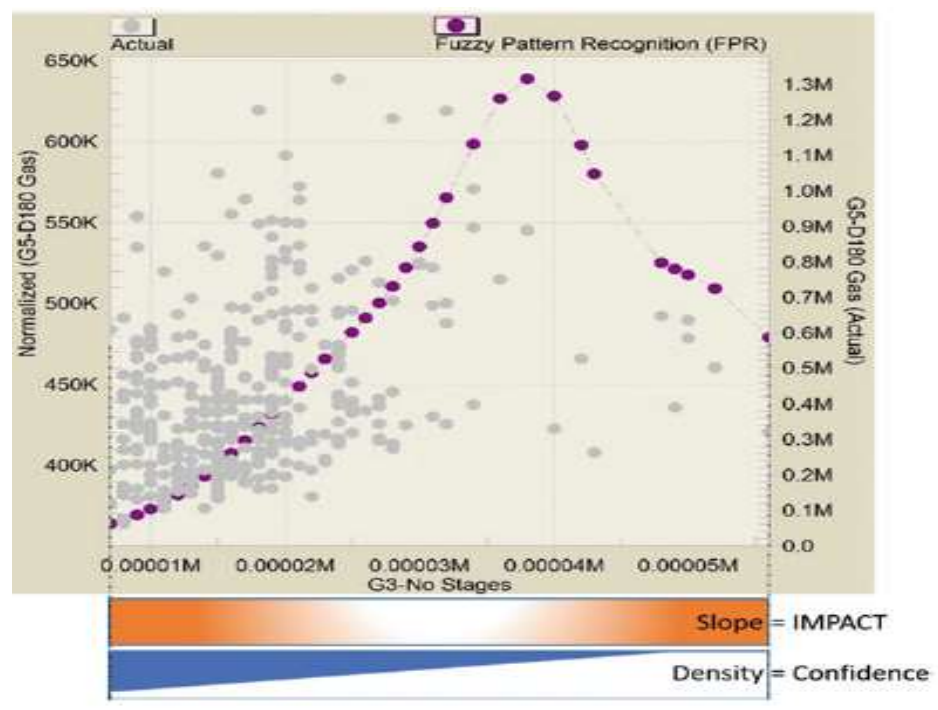

Figure 11 - Fuzzy Pattern Recognition [33] 


\section{Chapter 5: Results and Discussions}

The following section will outline the methodology, results, and discussions regarding the use of Al and machine learning's ability to identify shale anisotropy and implement this tool in optimizing completions designs. This will include all necessary background information, as well as many of the decisions made in order to complete this project.

\subsection{The Case Study: MSEEL MIP-3H}

The first step in completing this study was to select a well with an adequate dataset to be used for C-Means clustering, as well as the available data to validate the effectiveness of the modeling. Located in Morgantown, West Virginia, the Marcellus Shale Energy and Environmental Laboratory (MSEEL) is a multi-well public information database derived from 4 producing horizontal Marcellus shale gas wells and 1 pilot hole. The MSEEL project was sponsored by researchers at the National Energy Technology Laboratory (NETL) of the U.S. Department of Energy (DOE), West Virginia University, Ohio State University, Schlumberger, and the wells are operated by Northeast Natural Energy [35].

The project was created in order to provide a public database to provide better insight to Marcellus shale reservoir characterization and environmental health, with the purpose of promoting new and innovative practices to safely and cost effectively develop shale gas wells in the Marcellus. Some of the data available via MSEEL on a per well basis include: sidewall coring data, micro seismic, completions design and pump data, drilling data, mud logs, fiber optic DAS and DTS, fracture and geomechanical sonic scanner logs, and production logging [35]. 


\begin{tabular}{|c|c|c|}
\hline Logged Property: & Definition: & Calculated with: \\
\hline C11Elastic Modulus & Elastic Stiffness Coefficient in the C11 Direction & DT Compe ssional, DT Shear Fast and Slow \\
\hline C12 Elastic Modulus & Elastic Stiffness Coefficient in the C12 Direction & DT Compe ssional, DT Shear Fast and Slow \\
\hline C13 Elastic Modulus & Elastic Stiffness Coefficient in the C13 Direction & DT Compe ssional, DT Shear Fast and Slow \\
\hline C33Elastic Modulus & Elastic Stiffness Coefficient in the C33 Direction & DT Compe ssional, DT Shear Fast and Slow \\
\hline C44Elastic Modulus & Elastic Stiffness Coefficient in the C44 Direction & DT Compe ssional, DT Shear Fast and Slow \\
\hline C55Elastic Modulus & Elastic Stiffness Coefficient in the C55 Direction & DT Compe ssional, DT Shear Fast and Slow \\
\hline C66Elastic Modulus & Elastic Stiffness Coefficient in the C66 Direction & DT Compe ssional, DT Shear Fast and Slow \\
\hline Bore hole Deviation & Wellbore angular change from vertical (Inclination) & $\begin{array}{c}\text { Direct Measurement } \\
\end{array}$ \\
\hline DT Compressional & Measured travel time for compressional waves & Direct Measurement \\
\hline DIS Fast & Measured travel time for fast shear waves & Direct Measurement \\
\hline DTS Slow & Measured travel time for slow shear waves & Direct Measurement \\
\hline GR-Thompson & Originial measurement of gamma radition & Direct Measurement \\
\hline $\mathrm{GR}$ & Gamma Ray - Standard measurement of gamma radition & Direct Measurement \\
\hline Azi & Azimuth - Angular depature from true north & Direct Measurement \\
\hline Pore Pressure Gradient & Change in pore pressure per depth & Defined by tool operator \\
\hline Pore Pressure & Fluid pressure within the pore space of the rock & Pore Pressure Gradient \\
\hline PR Horiz & Poissons Ratio - Measure of material deformation perpendicular to applied force (Hoizontal) & DT Compe ssional, DT Shear Fast and Slow \\
\hline PR Vert & Poissons Ratio - Measure of material deformation perpendicular to applied force (Vetical) & DT Compe ssional, DT Shear Fast and Slow \\
\hline RHOB & Bulk Desnity - weight per given volume & Direct Measurement \\
\hline TVD & True Vertical Depth from surface reference & Borehole Deviation, Azimuth \\
\hline Iso Closure Stress Gradient & Closure stress per depth in Isotropic formations & Defined by tool operator \\
\hline Ansio Closure Stress Gradient & Closure stress per depth in Anisotropic formations & Defined by tool operator \\
\hline Iso Closure Stress & Pressure at which formation fractures close in Isotropic formations & Iso Closure Stress Gradient \\
\hline Aniso Closure Stress & Pressure at which formation fractures close in Anisotropic formations & Ansio Closure Stress Gradient \\
\hline Overburden Gradient & Overburden pressure per depth & Defined by tool operator \\
\hline Overburden Pressure & pressure resulting from the weight of overlying formations & Overburden Gradient \\
\hline YMDynamic $\mathrm{Hz}$ & Young's Modulus - moving average of formation tesnise stiffness in the horizontal direction & DT Compe ssional, DT Shear Fast and Slow \\
\hline YMStatic $\mathrm{Hz}$ & Young's Modulus - instantaneous tesnise stiffness in the horizontal direction & DT Compe ssional, DT Shear Fast and Slow \\
\hline YMDynamic Vt & Young's Modulus - moving average of formation tesnise stiffness in the vertical direction & DT Compe ssional, DT Shear Fast and Slow \\
\hline YMStatic Vt & Young's Modulus - instantaneous tesnise stiffness in the vertical direction & DT Compe ssional, DT Shear Fast and Slow \\
\hline
\end{tabular}

Table 3 - MIP-3H Sonic Scanner Data

Out of the four horizontal wells associated with the MSEEL project, the well MIP-3H was

selected. This was due to the fact that MIP-3H had the best data as it relates to this project. The

MIP-3H well was logged with the sonic scanner tool outlined in Table 2 of Section 1.3 and

repeated above as Table 3. In addition to the geomechanical log, MIP-3H's horizontal was also

logged with the Natural Fracture Log which provided the natural fracture count and the P32

natural fracture intensity [35]. These were all logs that were deemed to be potentially useful in

the process of identifying shale anisotropy and completion design optimization. Finally, MIP-3H

also had a production log on a per-cluster basis, which can be used to validate the hypothesis

that shale anisotropy has a significant impact on perforated clusters that produce zero gas. All

of these datasets are available to the public on the MSEEL website [35]. 


\subsection{Data Selection for C-Means Modeling}

Out of all of the logs that are available via the MSEEL project for well MIP-3H, a certain number of logs need to be identified as the optimal combination for C-Means fuzzy clustering. The first step in order to achieve this goal is to identify the parameters from the sonic scanner $\log$ and the natural fracture log that have the strongest correlation to production. This is done by replicating the Well Quality Analysis (WQA) methodology outlined in section 3.2. In development, WQA is applied to a series of well's production values. In theory, it can be applied on a much smaller scale by finding a logged parameters impact to production on a per cluster basis.

The production is taken from the production log for MIP-3H. To note: the sonic scanner log does not begin logging data until 7800' measured depth, as a result the first cluster in the production log was created outside of the available range of data. For the purposes of this study, that cluster is ignored and stage 28 is taken to have 3 clusters instead of 4 . Similarly, all of the clusters for Stage 1 are grouped together in terms of reported production. As a result, stage 1 and its clusters are completely ignored. The result is 126 clusters ranging between stage 28 and stage 2.

Each cluster is reported as having a 2 -ft range of impact area where the casing was perforated, and the corresponding measured production for that cluster. To account for the $2-\mathrm{ft}$ range relative to each value, the measurements from the sonic scanner and natural fracture logs are averaged within that 2-ft interval. All other data outside of the 2-ft intervals for the 126 
clusters are ignored. The result is 126 averaged values for each log that correspond to the MD of the clusters on the production log.

Following the steps outlined in the WQA, this method will be referred to as a simply a single well quality analysis (SQA) due to the lack of multi-well production. The first step in preforming the SQA was to identify the fuzzy boundaries that accurately reflect the per-cluster set of production data. First, the average across all clusters was found to be $41.03 \mathrm{mcfd}$. This was then chosen to be the centroid for the 'average' cluster production. The production ranged from $0 \mathrm{mcfd}$ to $200 \mathrm{mcfd}$ per cluster. As a result, these are the chosen boundaries for the minimum of the 'poor' class and maximum of the 'good' class respectively. The other boundaries were selected based on creating a frequency distribution of the production data to evenly describe the data and set the remaining bounds. This histogram is presented as Figure 12.

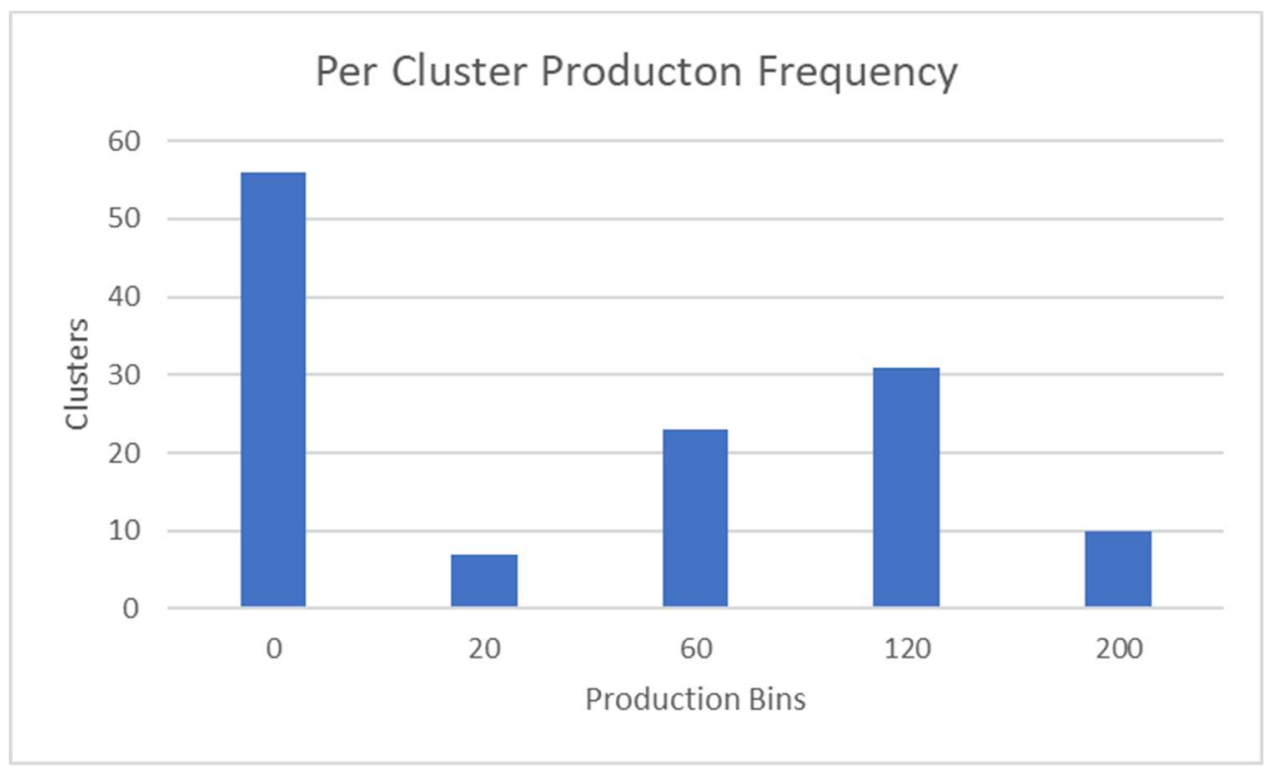

Figure 12 - Per Cluster Production Frequency Distribution 
The poor class was given only 0 mcfd production a full membership value of 1.0. Full membership to the 'average' class ranged between 20-60 mcfd and membership to the 'good' class ranged from 120-200 mcfd based on the distribution of the production log data. All other values were considered fuzzy based on the fuzzy logic theory described in section 3.2. The table summarizing these decisions and the SQA plot for per cluster production are shown below as Table 4 and Figure 13 respectively.

\begin{tabular}{|c|c|c|c|c|c|c|c|c|}
\hline \multicolumn{3}{|c|}{ Poor Class } & \multicolumn{3}{c|}{ Average Class } & \multicolumn{4}{c|}{ Good Class } \\
\hline Set & Production & Membership & Set & Production & Membership & Set & Production & Membership \\
\hline A & 0 & 0 & A & 0 & 0 & A & 60 & 0 \\
\hline B & 0 & 1 & B & 20 & 1 & B & 120 & 1 \\
\hline C & 0 & 1 & C & 60 & 1 & C & 200 & 1 \\
\hline D & 20 & 0 & D & 120 & 0 & D & 200 & 0 \\
\hline
\end{tabular}

Table 4 - SQA Fuzzy Logic Definitions for Cluster Production

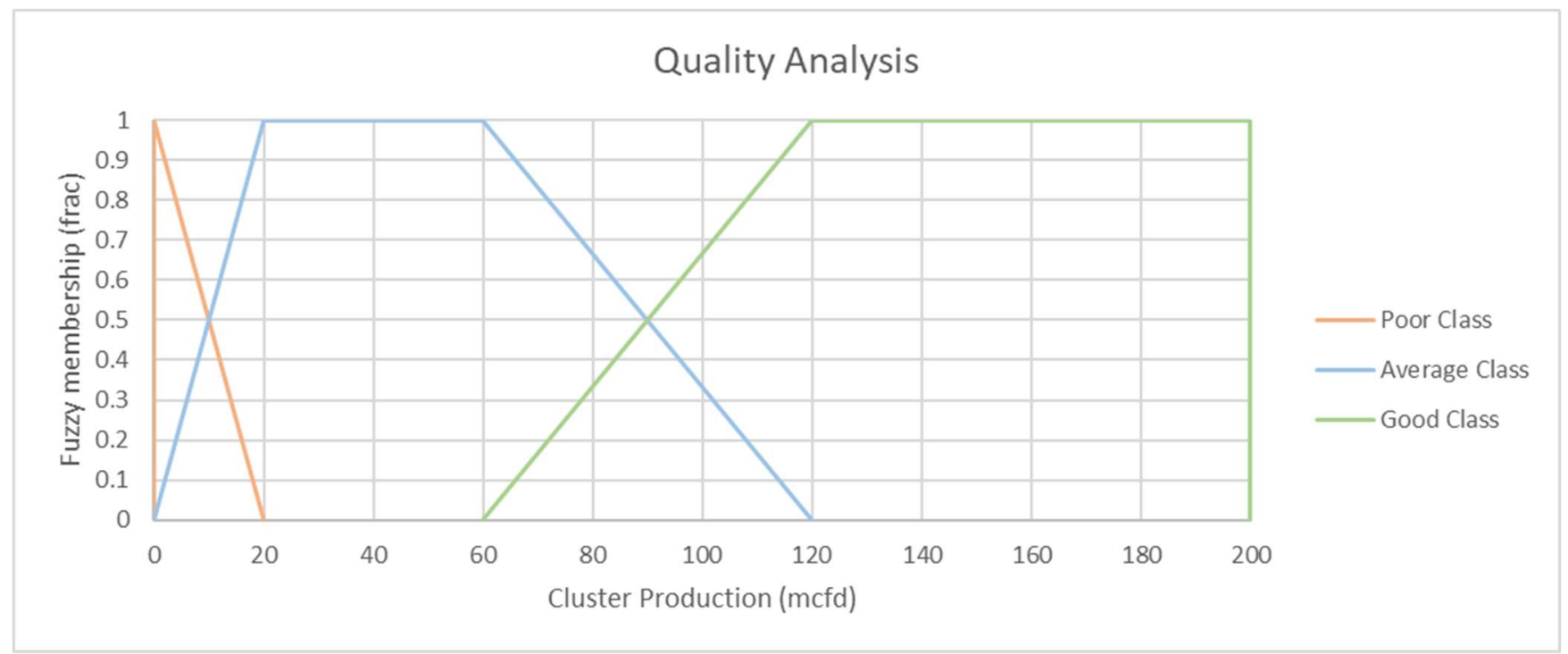

Figure 13 - QA Fuzzy Logic Per-Cluster Production Plot

Using this plot, the membership value for each cluster's production can be found either directly for full membership, or by using linear interpolation to find partial memberships. Once the class membership to each cluster's production is known, the methodology of WQA can be 
followed in order to determine a given parameter's impact on the production. First select one of the available logs to be analyzed. For this methodology, the sonic log DT Shear Fast from the sonic scanner set of logs will be discussed. This log data has been refined to represent the average logged value over each cluster impact area. This value is multiplied by the class membership of the production, in order to come up with a relative membership value for the logged variable itself. This method is repeated for the log's value at each cluster, so there is a table of 'poor' 'average' and 'good' DT Shear Fast values at every cluster. These values are summed across all clusters to have a total 'poor' 'average' and 'good' DT Shear Fast value that is divided by the total production membership values for the 'poor' 'average' and 'good' classes as shown in Equation 4. The results shown in Table 5 are the weighted average values of DT Shear Fast in the 'poor' 'average' and 'good' production classes.

Table 5 demonstrates the resulting QA values for the log DT Shear Fast and its corresponding plot is shown as Figure 14:

\begin{tabular}{|c|c|}
\hline Classification & Calculauted Value \\
\hline Poor & 120.368322 \\
\hline Average & 120.4483309 \\
\hline Good & 121.0439063 \\
\hline
\end{tabular}

Table 5 - QA Results for DT Shear Fast 


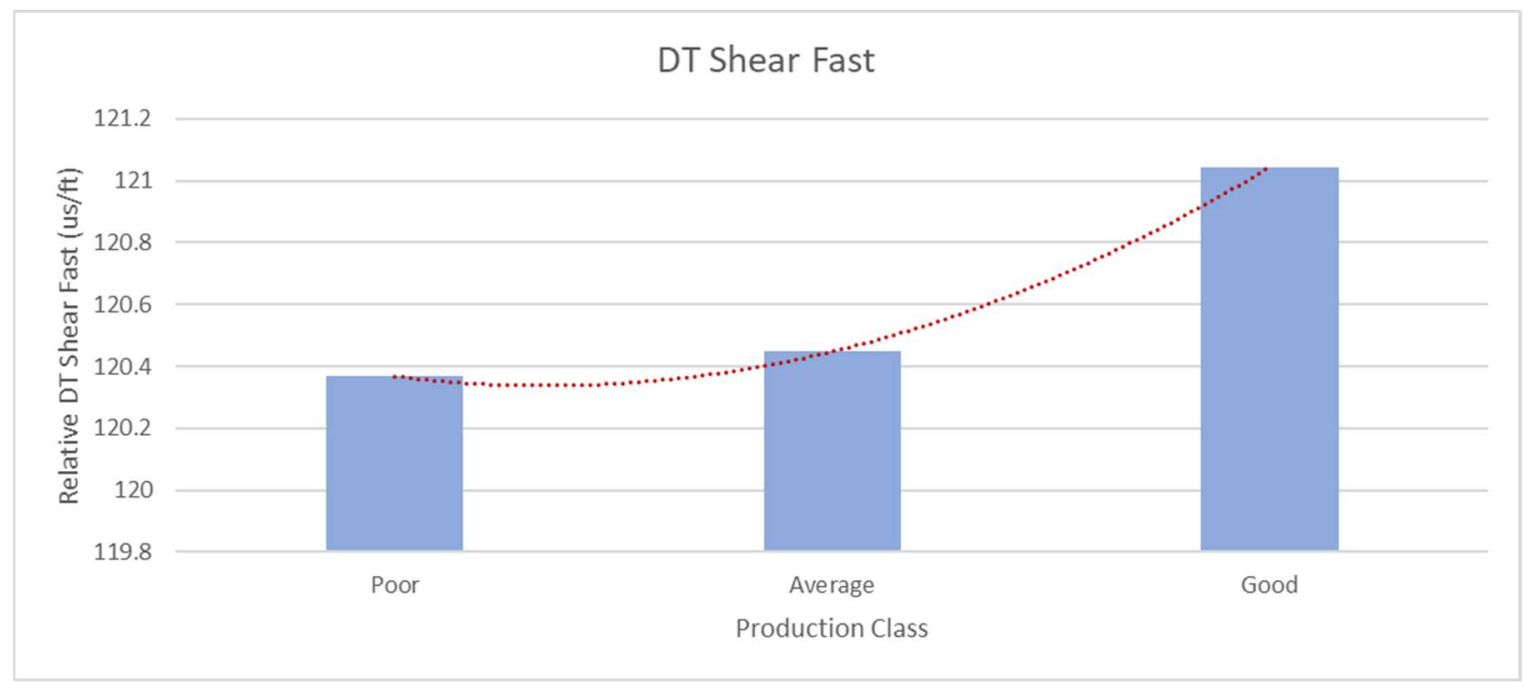

Figure 14 - QA Plot for DT Shear Fast

The QA results for DT Shear Fast show a strong positive correlation between the DT Shear Fast log and the production log. This is indicated because of the clear positive trend (slope) between the three data-points. Not all logs displayed a clear positive trend with production. For example, the bulk density (RHOB) displayed a clear negative trend with the production log as shown below in Figure 15:

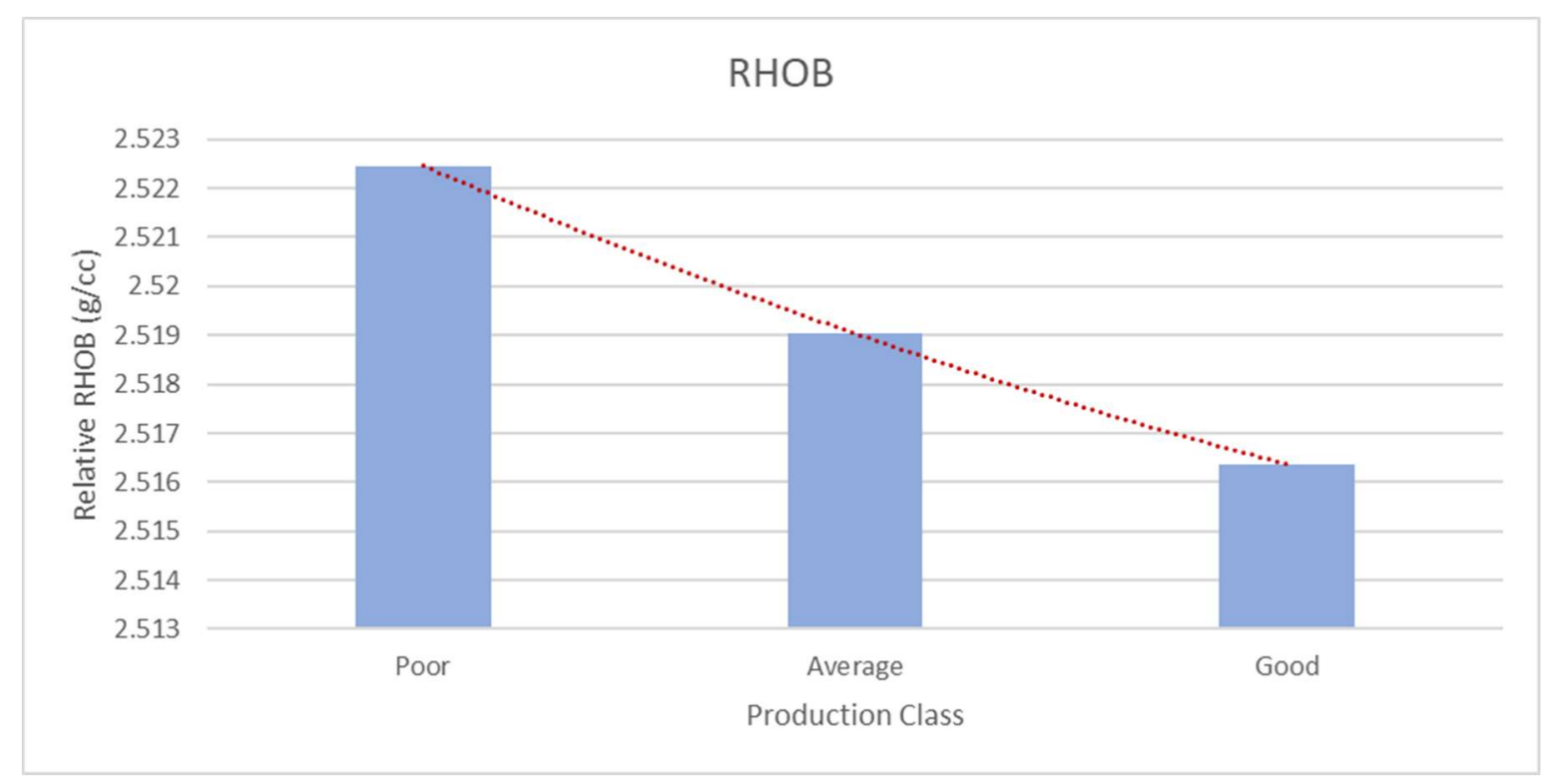

Figure 15 - QA Plot for RHOB 
Although the QA trend for RHOB is negative, it is abundantly clear that there is still a strong trend between bulk density and the production. The slope of these plots suggests the magnitude of correlation, and the sign of the slope indicates the correlations direction. Some parameters did not show as clear of a trend as DT Shear Fast and RHOB. One such parameter was the natural fracture intensity, P32, from the fracture log. This plot is presented below as Figure 16:

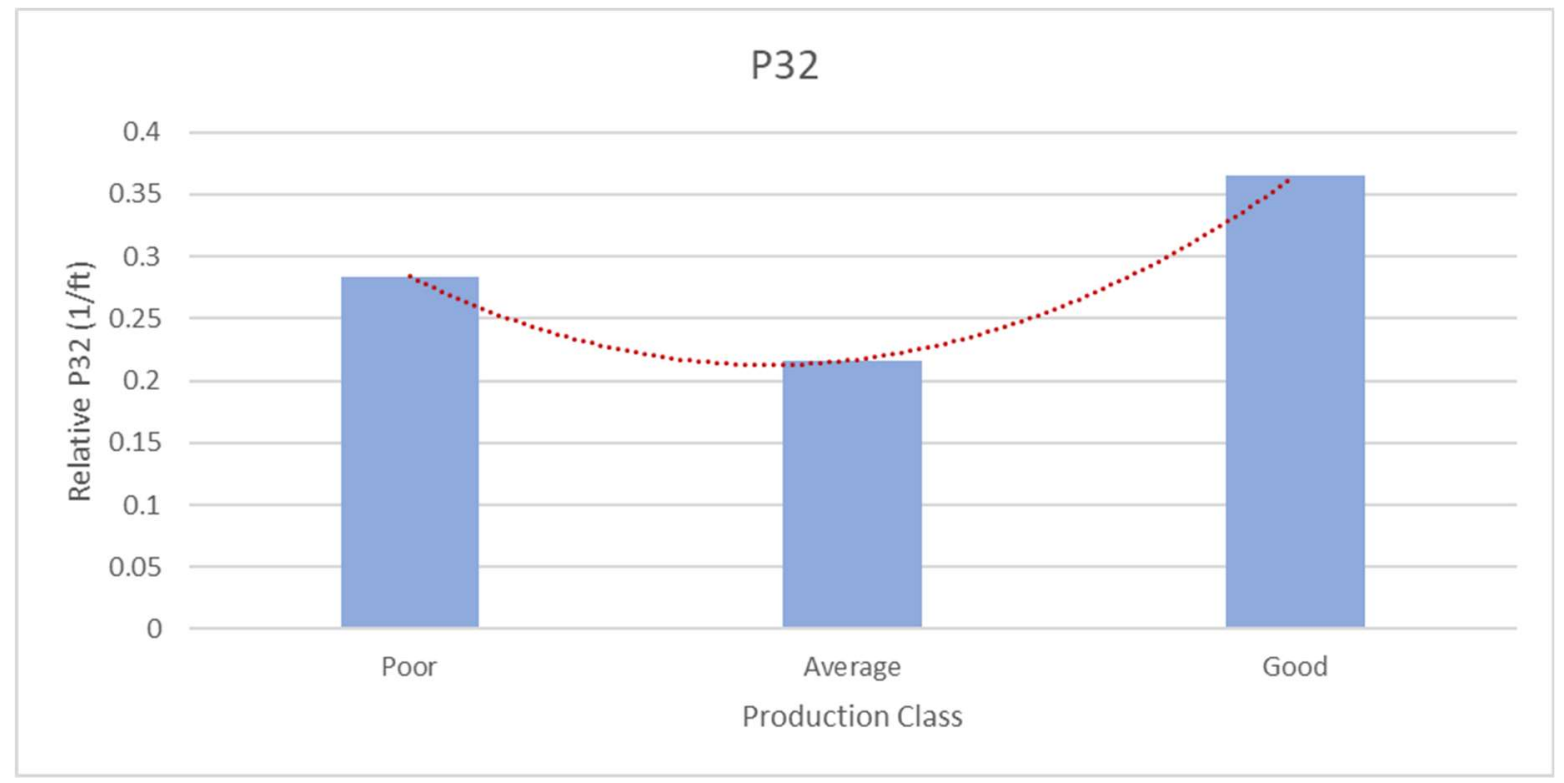

Figure 16 - QA Plot for P32

The trend generated by P32 is overall positive, by taking the sum of the slopes between the points 'poor' and 'average' as well as 'average' and 'good'. This indicates there still make be a correlation between P32 and production, but that is simply not a strong as with parameters such as DT Shear Fast and RHOB. 
To better provide a clear picture as to how these plots relate to the minimum,

maximum, and average values for each measured property, Table 6 was generated to

summarize these findings. Based on Table 6, the 'poor', 'average', and 'good' values can be

related to what was the overall average for the dataset.

\begin{tabular}{|c|c|c|c|c|}
\hline Logged Property: & Minimum & Maximum & Average & Units \\
\hline C11 Elastic Modulus & 6.084528 & 13.41108 & 7.214452 & Mpsi \\
\hline C12 Elastic Modulus & 1.6342104 & 7.141125 & 2.529266 & Mpsi \\
\hline C13 Elastic Modulus & 1.6342104 & 7.141125 & 2.529266 & Mpsi \\
\hline C33 Elastic Modulus & 3.5361262 & 12.058561 & 4.788259 & Mpsi \\
\hline C44 Elastic Modulus & 1.1677492 & 2.557899 & 1.36333 & Mpsi \\
\hline C55 Elastic Modulus & 1.1677492 & 2.557899 & 1.36333 & Mpsi \\
\hline C66 Elastic Modulus & 2.1133164 & 3.1349774 & 2.342593 & Mpsi \\
\hline Borehole Deviation & 77.344254 & 92.125746 & 90.4726 & deg \\
\hline DT Compressional & 53.14632 & 73.774774 & 68.82954 & $\mathrm{us} / \mathrm{ft}$ \\
\hline DTS Fast & 109.4331 & 125.2704 & 120.5123 & $\mathrm{us} / \mathrm{ft}$ \\
\hline DTS Slow & 121.53614 & 168.3933 & 158.2419 & $\mathrm{us} / \mathrm{ft}$ \\
\hline GR-Thompson & 0.1167357 & 0.5490429 & 0.363082 & unitless \\
\hline GR & 184.19464 & 525.34986 & 420.7829 & api \\
\hline Azi & 321.45506 & 327.1445 & 323.9164 & deg \\
\hline Pore Pressure & 4819.414 & 4864.7306 & 4841.001 & psi \\
\hline PR Horiz & 0.1560536 & 0.3151008 & 0.200893 & unitless \\
\hline PR Vert & 0.2070501 & 0.3452657 & 0.256878 & unitless \\
\hline $\mathrm{RHOB}$ & 2.4466908 & 2.7823518 & 2.520145 & $\mathrm{~g} / \mathrm{cc}$ \\
\hline TVD & 7414.4832 & 7484.201 & 7447.694 & $\mathrm{ft}$ \\
\hline Iso Closure Stress Gradient & 0.8280159 & 1.0229019 & 0.899041 & $\mathrm{psi} / \mathrm{ft}$ \\
\hline Ansio Closure Stress Gradient & 0.8943831 & 1.031129 & 0.951357 & $\mathrm{psi} / \mathrm{ft}$ \\
\hline Iso Closure Stress & 6164.0166 & 7616.2186 & 6695.82 & psi \\
\hline Aniso Closure Stress & 6632.9846 & 7677.4762 & 7085.479 & psi \\
\hline Overburden Pressure & 8630.4586 & 8711.6096 & 8669.116 & psi \\
\hline YM Dynamic Hz & 4.9891146 & 8.2523354 & 5.627628 & Mpsi \\
\hline YM Static $\mathrm{Hz}$ & 2.8282852 & 5.2758758 & 3.284704 & Mpsi \\
\hline YM Dynamic Vt & 2.7842638 & 7.087161 & 3.463914 & Mpsi \\
\hline YM Static Vt & 1.3740678 & 4.3752898 & 1.80418 & Mpsi \\
\hline Count Sum & 0 & 1.4 & 0.231747 & unitless \\
\hline P32 & 0 & 1.7713714 & 0.272091 & $1 / \mathrm{ft}$ \\
\hline
\end{tabular}

Table 6 - Min, Max, and Average of Per Cluster Logged Properties

In order to compare and contrast the QA results across all of the well logs, the results as

presented in Table 5 must be normalized. The normalization is done by taking the smallest

value as zero and the largest value is set as 1 . The middle value is then calculated using linear

interpolation. The sum of the slopes between the points 'poor' and 'average', and 'average' and

'good' is referred to as the "magnitude". This is how strong the correlation is between the log 
and the production. For example, DT Shear Fast has a magnitude of +1 , indicating a strong positive relationship. $\mathrm{RHOB}$ would have a magnitude of -1 , which is a strong negative relationship. The normalized QA for P32 is shown below in Table 7 as an example of these results. Note that the QA magnitude for P32 is between 0 and 1, and has a positive value following the trends seen for P32 in Figure 16.

\begin{tabular}{|c|r|}
\hline \multicolumn{2}{|c|}{ QA (Normalized) } \\
\hline Poor & 0.452133171 \\
\hline Average & 0 \\
\hline Good & 1 \\
\hline Magnitude & 0.547866829 \\
\hline
\end{tabular}

Table 7 - Normalized QA Values for P32

Table 8 summarizes the magnitudes, reported as a degree of influence in degrees, for all of the available logs from the sonic scanner and natural fracture logging tools. There is the normalized degree of influence for each value, and the absolute degree of influence which is simply the absolute value. The direction of influence is not relevant in the scope of the study, just the idea that there is a high magnitude of influence in general. 


\begin{tabular}{|c|c|c|}
\hline Log & Normalized Degree of Influence & Absolute Degree of Influence \\
\hline DT Compressional & $100 \%$ & $100 \%$ \\
\hline DT Shear Fast & $100 \%$ & $100 \%$ \\
\hline RHOB & $-100 \%$ & $100 \%$ \\
\hline C11 & $-100 \%$ & $100 \%$ \\
\hline C12 & $-100 \%$ & $100 \%$ \\
\hline $\mathrm{C} 13$ & $-100 \%$ & $100 \%$ \\
\hline C33 & $-100 \%$ & $100 \%$ \\
\hline C66 & $-100 \%$ & $100 \%$ \\
\hline Borehole Deviation & $-100 \%$ & $100 \%$ \\
\hline YM Dynamic Horiz & $-100 \%$ & $100 \%$ \\
\hline YM Static Horiz & $-100 \%$ & $100 \%$ \\
\hline YM Static Vert & $-98 \%$ & $98 \%$ \\
\hline YM Dynamic Vert & $-96 \%$ & $96 \%$ \\
\hline $\mathrm{C} 44$ & $-95 \%$ & $95 \%$ \\
\hline $\mathrm{C} 55$ & $-95 \%$ & $95 \%$ \\
\hline Dt Shear Slow & $81 \%$ & $81 \%$ \\
\hline PR Vertical & $-78 \%$ & $78 \%$ \\
\hline TVD & $-70 \%$ & $70 \%$ \\
\hline Overburden Pressure & $-70 \%$ & $70 \%$ \\
\hline Pore Pressure & $-70 \%$ & $70 \%$ \\
\hline Iso Closure Stress & $-67 \%$ & $67 \%$ \\
\hline Iso Closure Stress Gradient & $-66 \%$ & $66 \%$ \\
\hline Count Sum & $65 \%$ & $65 \%$ \\
\hline PR Horizontal & $-59 \%$ & $59 \%$ \\
\hline P32 & $55 \%$ & $55 \%$ \\
\hline Aniso Closure Stress & $-47 \%$ & $47 \%$ \\
\hline Aniso Closure Stress Gradient & $-44 \%$ & $44 \%$ \\
\hline Azimuth & $-42 \%$ & $42 \%$ \\
\hline $\mathrm{GR}$ & $-31 \%$ & $31 \%$ \\
\hline GR Thompson & $9 \%$ & $9 \%$ \\
\hline
\end{tabular}

Table 8 - QA Summary (Green: high degree of influence, Yellow: manipulated data with high degree of influence, Red: low degree of influence)

The information prepared in Table 8 can also be presented in a tornado chart, showing the logs and their absolute degree of influence as in Figure 16. 


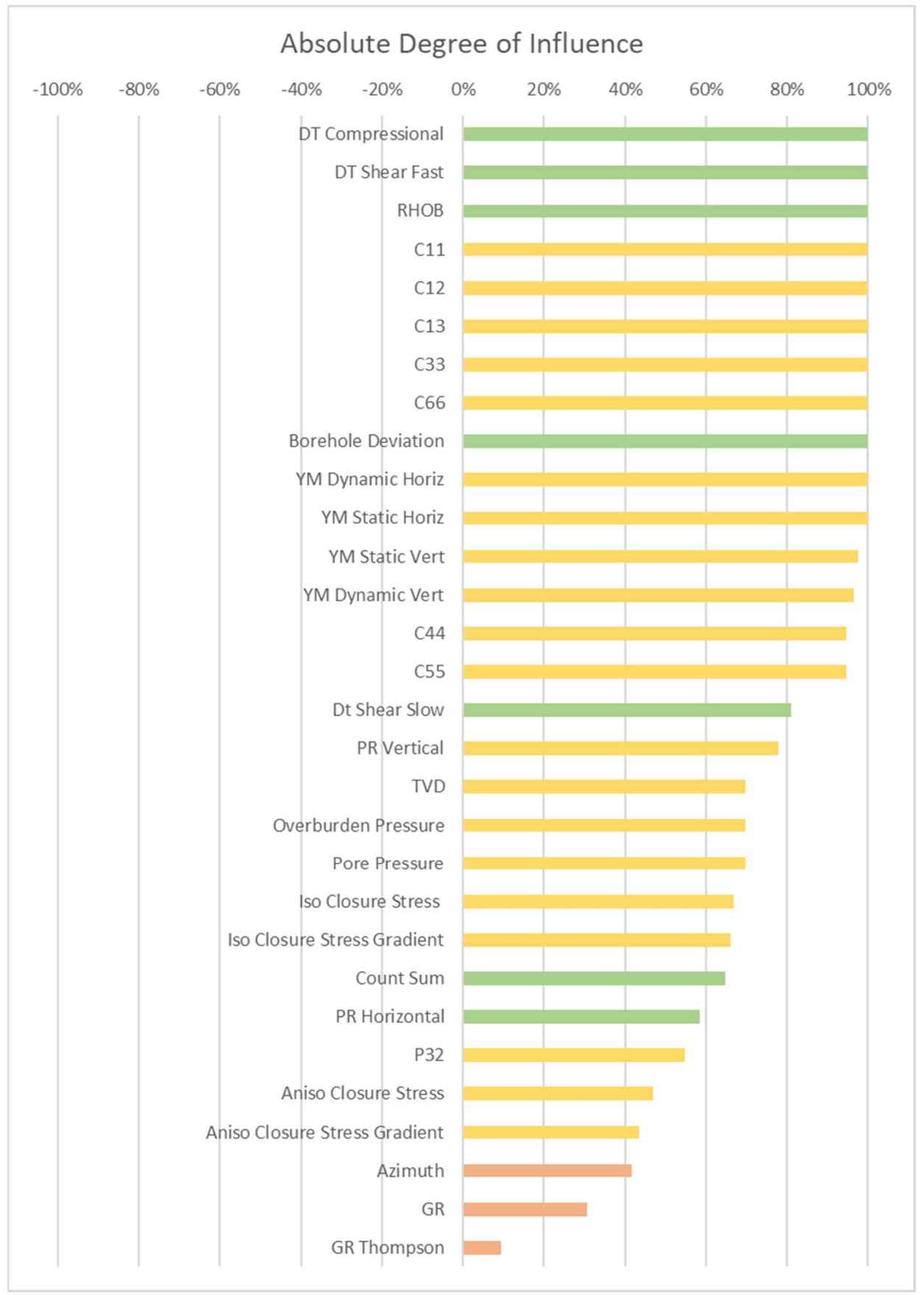

Figure 17 - QA Summary Tornado Chart (Green: high degree of influence, Yellow: manipulated data with high degree of influence, Red: low degree of influence)

The color schemes presented in Table 8 and Figure 17 are: green values are found to be raw measured data with a high degree of influence, yellow values are found to be calculated or manipulated data with a high degree of influence, and red values are found to be raw measured 
data with a low degree of influence. All of the additional QA plots for every other parameter can be found in the appendix of this paper.

The data selected for the C-Means fuzzy clustering are the green logs, where the data is raw measured data that is unmanipulated with a high degree of influence on production.

Although the yellow logs demonstrated a high degree of influence, the data has been calculated based on measurements from other logs using proprietary equations or was interpreted and reported by human work. Selecting raw and unmanipulated data is one of the tenants to using a successful data-driven Al and ML algorithm, which was outlined in Chapter 3. It is vital to the success of the study to have data that is shown to have an impact on production, as well as being raw measured data that remains true to the functional requirements for C-Means Fuzzy Clustering. A summary of the selected data derived from Table 3 is presented below as Table 9.

\begin{tabular}{|c|c|c|}
\hline Logged Property: & Definition: & Calculated with: \\
\hline DT Compressional & Measured travel time for compressional waves & Direct Measurement \\
\hline DTS Fast & Measured travel time for fast shear waves & Direct Measurement \\
\hline DTS Slow & Measured travel time for slow shear waves & Direct Measurement \\
\hline Borehole Deviation & Wellbore angular change from vertical (Inclination) & Direct Measurement \\
\hline RHOB & Bulk Desnity - weight per given volume & Direct Measurement \\
\hline Count Sum & Number of natual fractures within a given interval (natural fracture log) & Direct Measurement \\
\hline
\end{tabular}
Table 9 - Summary of Selected Data

\subsection{Using C-Means Fuzzy Clustering to Identify Shale Anisotropy}

Now that the optimum set of data has been selected to be passed through the C-Means fuzzy clustering algorithm, the next step is to preprocess the data using python. The data is preprocessed using a min-max function, which normalizes each log to its lowest value equaling zero and its largest value equaling one. This will allow the C-Means algorithm to accurately compare the data to each other in the same scale in order to find patterns and trends, without being thrown off by the magnitude of a given logs value. For example, the gamma ray log may 
have recorded values in the hundreds, whereas the numerical representation for a log such as bulk density is in single digits. This difference in numerical magnitude, largely caused by units, is dealt with by normalizing all of the logs with the min-max function.

The data that is passed through the C-Means algorithm is averaged relative to the measured depth for each cluster as presented on the production log. This is done to be able to compare the results of the C-Means fuzzy clustering directly to the 126 clusters available on the production log. The number of centroids chosen to be passed through the C-Means algorithm was three. This was to increase the granularity in order to detect the changes between the rock type. The algorithm can find the optimum number of centers using the fuzzy partition coefficient (FPC) and plotting the FPC for a different number of centroids. The FPC describes how cleanly the data is being represented, by how easily the algorithm can assign centroid locations [36]. When the dataset is large and multidimensional, the FPC has a negative linear relationship to the number of cluster centers. Meaning the optimum number is two, and the FPC decreases with time. However, an FPC greater than 0.5 is still acceptable to use for good results. Figure 18 shows the FPC vs number of clusters plot for this study:

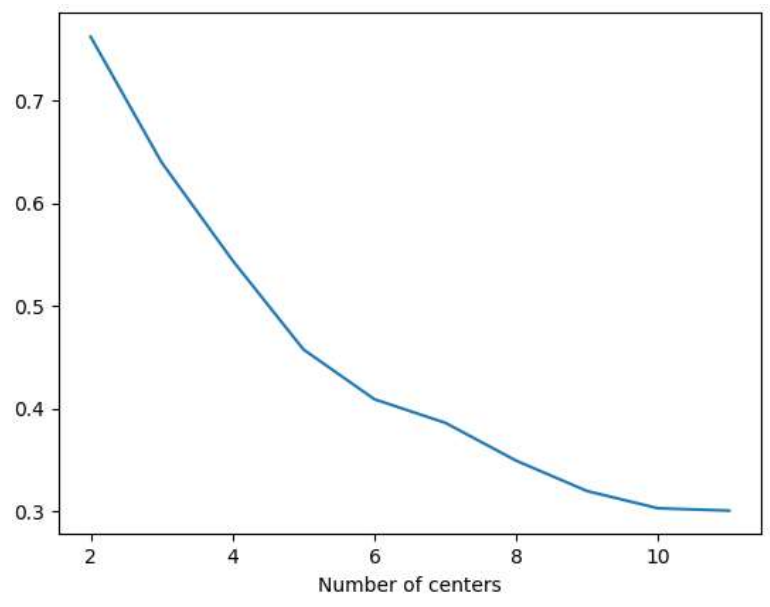

Figure 18 - FPC vs Number of Cluster Centers 
Although the optimum number of clusters for this dataset is 2 , the number of centers of 3 was chosen to attempt to increase the number of rock classifications in order to better identify anisotropy. The FPC for 3 centers was 0.65 , which is still a good value for this study. Now the data set outlined in Table 9, averaged on a per cluster basis, and the desired number of centers to be 3 are passed through the C-Means fuzzy clustering algorithm. The full algorithm used in this study can be found in the Appendix. The output is a fuzzy class assignment to classes 0,1 , and 2 for every cluster.

In order to identify the changes in rock properties, or the membership to each of the three classes, a new parameter was created. The Anisotropy Indicator Value (AIV) measures the change in membership from one cluster to another. In this context, it is the change in class membership between one cluster to another within a given stage due to the plug-and-perf completions design, which is isolating stages from one another. Clusters are not related to clusters in a different stage using AIV. As a result, 3-4 clusters (depending on the number in a given stage) are related to the cluster before it. The last cluster is related to the one behind it. The Equation 5 represents how AIV is calculated, where $\mathrm{C}$ is the class membership, $\mathrm{i}$ is the class assignment, and $\mathrm{n}$ is the cluster number that the data is relative to:

$$
A I V=\sum\left|C_{i}-C_{i_{n+/-1}}\right|
$$

Equation 5 - Anisotropy Indicator Value (AIV)

The AIV is then calculated for the class assignments at every cluster in order to quantify the change, or the anisotropy, at the locations of every cluster in the horizontal. The AIV calculation uses the absolute value of the difference between class memberships, since the 
magnitude of the change is what is important when assessing anisotropic behavior, not the direction of the change. The following example for calculating the AIV between two clusters is as follows: cluster $\mathrm{x}$ has a membership value of 0.45 for class $0,0.45$ with class 1 , and 0.1 with class 2 while cluster y has a membership of 0.9 for class $0,0.06$ with class 1 , and 0.04 with class 2. The AIV for this example is calculated using Equation 5:

$$
A I V_{x y}=\sum\left|C_{x}-C_{y}\right|=|0.45-0.9|+|0.45-0.06|+|.1-0.04|=0.90
$$

With the AIV, the membership values to each class, and the production at every cluster, some plots can be generated in order to interpret the results. The first such plot is plotting the membership values of each class vs production, with the last cluster of each stage marked with a dot. This is presented below for clusters 20-24 in Stage \#6 in Figure 19: 


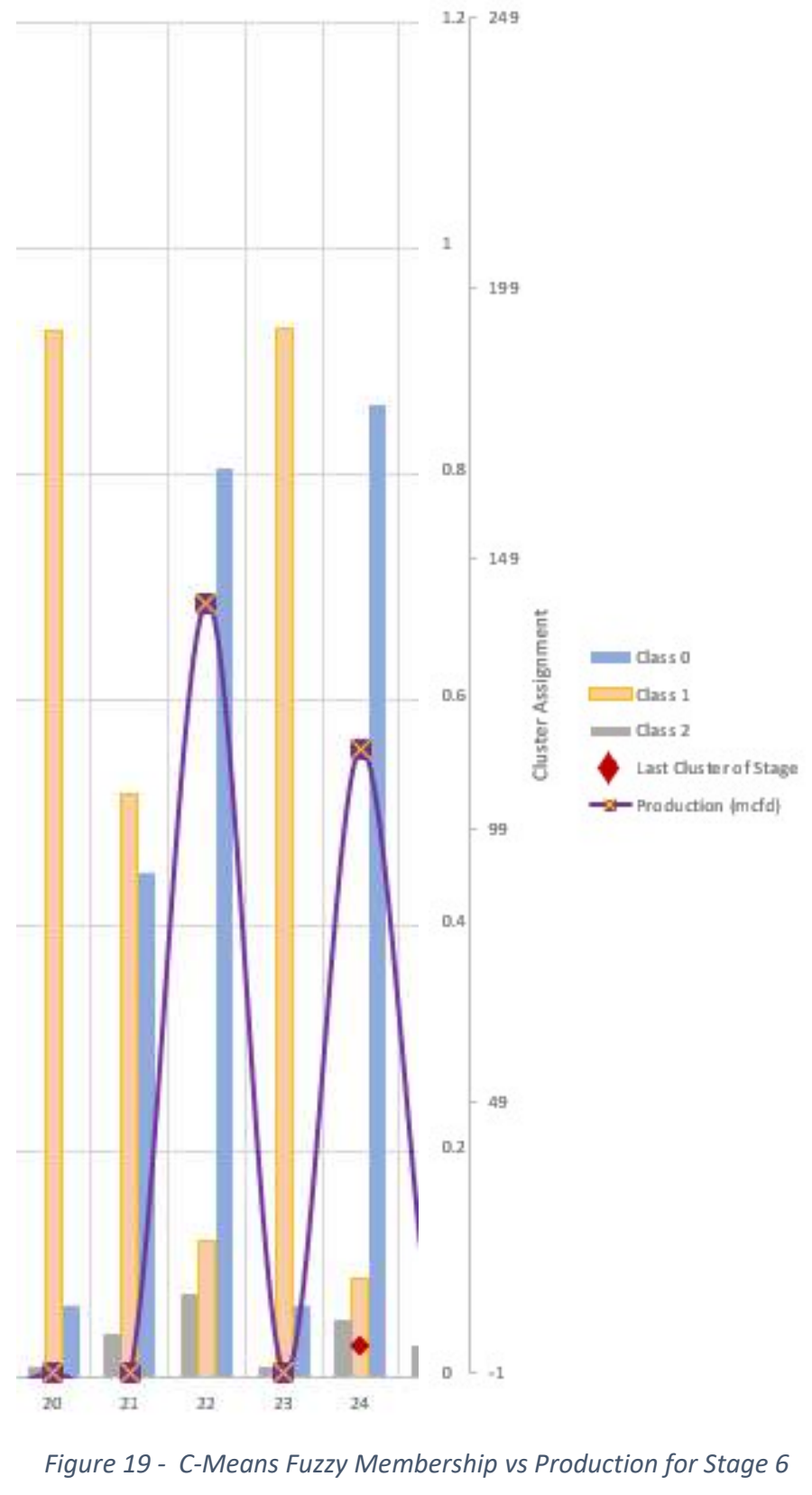

The correlation between the clusters with zero production and clusters with the Class 1 membership in this stage is apparent. The clusters with a Class 0 assignment both produce gas. This suggests that fractures propagated in this stage within rock belonging to Class 0 , but where there was anisotropy evident by a large difference in class membership between Class 0 and Class 1 there was zero production. Fractures did not propagate in Stage 6 where there were 
two distinct classes of rock type. The fractures propagated into one class of the shales but not into the other class, where there was a huge swing of anisotropy.

When plotting the AIV vs Production for Stage 6, it can be observed that the AIV remains high (above the average AIV for all 126 clusters) where there is no production. Note that the AIV is high at clusters 22 and 24 due to the significant anisotropy at cluster 23 , as both clusters are related to cluster 23 . Clusters 21 and 22 are still above the average AIV for the entire lateral.

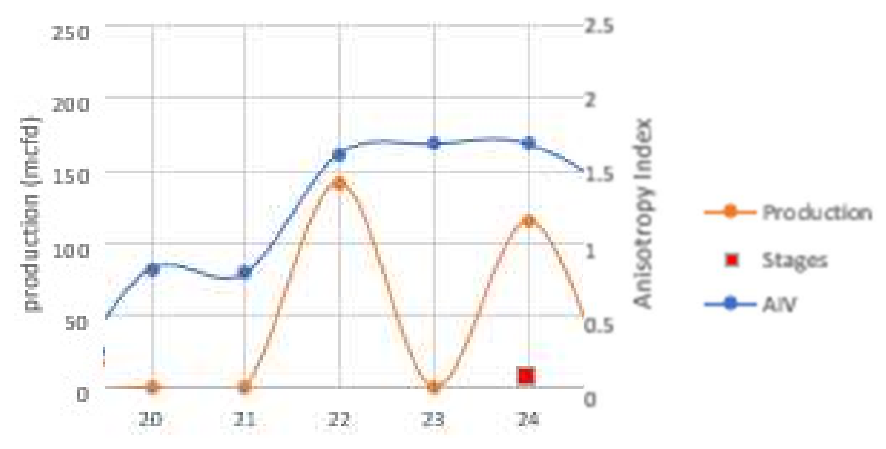

Figure 20 - Stage 6 AIV vs Production

These trends of high anisotropy, observed via the changes in lithological classification at every cluster, resulting in zero production are evident throughout the wellbore. The examples presented in Figures 19 and 20 demonstrated the anisotropy only within Stage 6. The following examples will demonstrate anisotropy in Stage 13, which was so severe only 1 cluster produced gas. Figure 21 shows the C-Means fuzzy membership vs production plot for Stage 13: 


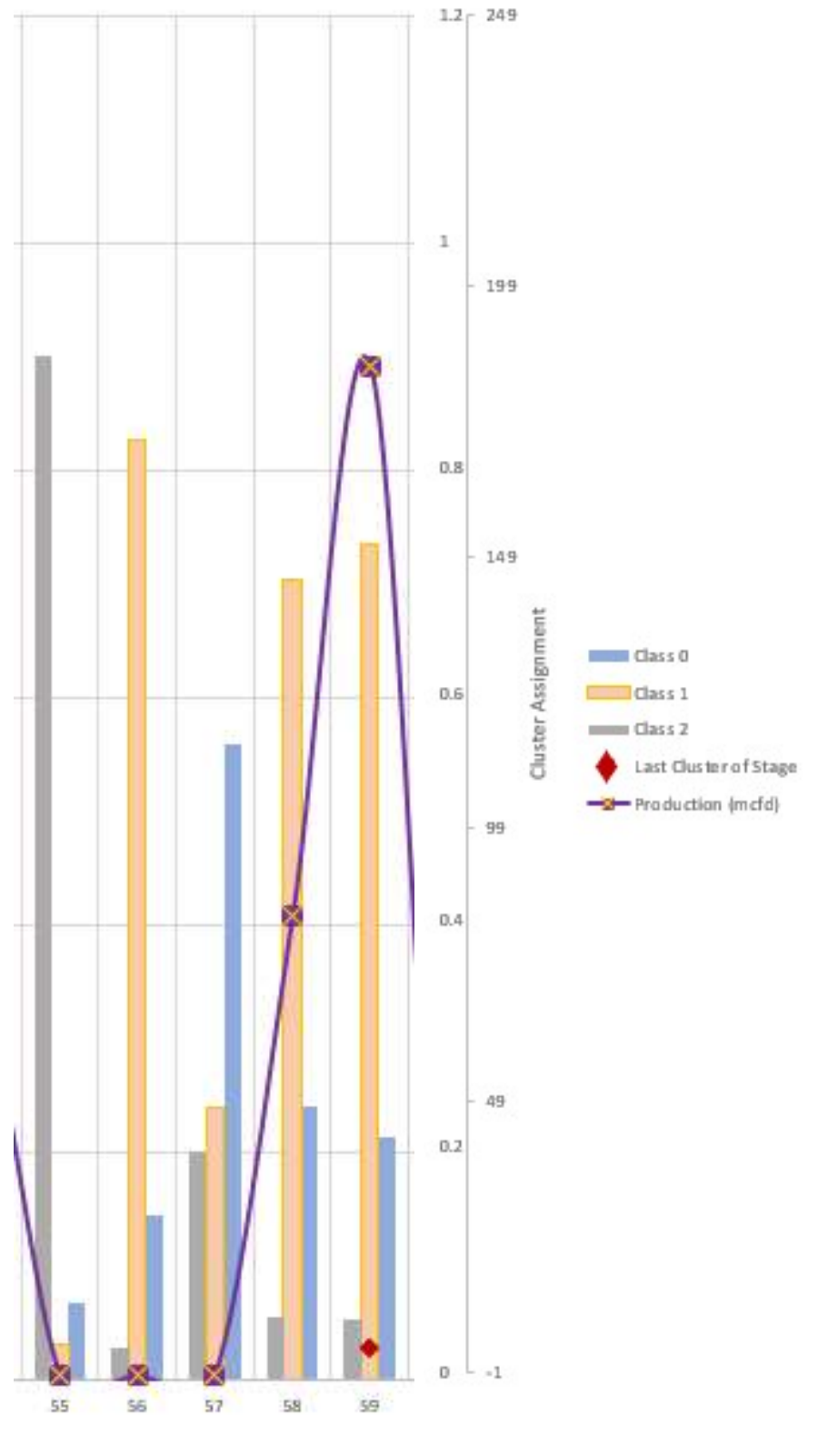

Figure 21 - C-means Fuzzy membership vs Production for Stage 13

It was observed that the dominant class at cluster 57 switches to Class 0, cluster 56 switches back to Class 1 , and cluster 55 is dominated by Class 2 . This resulted in significant anisotropy for this staged, which observed zero production for those three clusters. The two clusters that produced gas, 58 and 59, where next to each other with a similar fuzzy class 
membership which suggest a similar shale lithology at those points. Cluster 56 also had a similar class membership to the producing clusters but was between two clusters of radically different class membership. This suggests that when fracturing stage, having significant anisotropy around a cluster could contribute to zero production. When analyzing which dominant class of shales tends to have the most production, there was no conclusive answer. The results of the study suggest that successful fracture propagation is independent of being at a specific class, rather that clusters with similar classifications will fracture when grouped together whereas ones with different geomechanical properties within the same stage will not.

When plotting Stage 13's AIV vs Production, the impact of a high AIV on the production is clear. This is shown in Figure 22, were the AIV is well below average for the two producing clusters, and the AIV is well above average for the three clusters that are producing no gas.

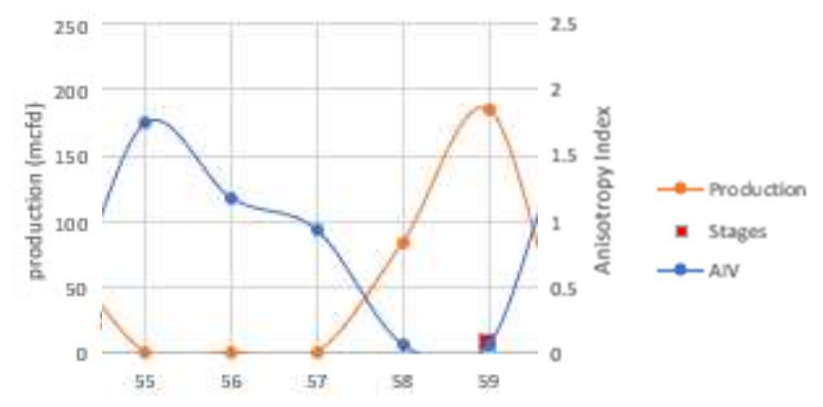

Figure 22 - AIV vs Production for Stage 13

This section of the report contains figures from only two stages to clearly present and explain the results of the C-Means Fuzzy Clustering. The entire C-Means Fuzzy Membership vs Production and AIV vs Production plots for all 27 stages available to this study from MIP-3H are available in the Appendix under "MIP-3H Per-Cluster Classification Plots". 
The focus of this part of the project was to determine whether or not shale anisotropy, identified using C-Means Fuzzy Clustering, had a direct input on the 56 out of 126 clusters that produced zero gas. By comparing the AIV to the production, it was determined that 49 out of the 56 clusters producing zero gas occurred in areas of high anisotropy, as was detailed with Stages 6 and 13 above. It was determined that if a cluster had an AIV value that was greater than the average AIV for all 126 clusters, that the cluster had zero production due to shale anisotropy at that cluster. Table 10 summarizes the findings, where it was determined $88 \%$ of the clusters that produced zero gas happened in areas of significant anisotropy.

\begin{tabular}{|c|c|c|c|}
\hline Total Clusters & Zero Production & Result of Anisotropy & Percentage \\
\hline 127 & 56 & 49 & $88 \%$ \\
\hline
\end{tabular}

Table 10 - Breakdown of Non-producing Clusters due to Anisotropy

These results can also be visualized by plotting the cluster efficiency of a given stage vs the average AIV of the stage. The cluster efficiency is simply the number of clusters which produce gas divided by the total number of clusters within a stage. This plot is presented below as Figure 23, where the cluster efficiency axis is inversed to show the correlation between high AIV and low cluster efficiency on a per-stage basis. This plot further supports the idea that there is an impact on cluster-performance within a stage due to the shale anisotropy and heterogeneity within the isolated section of the wellbore. 


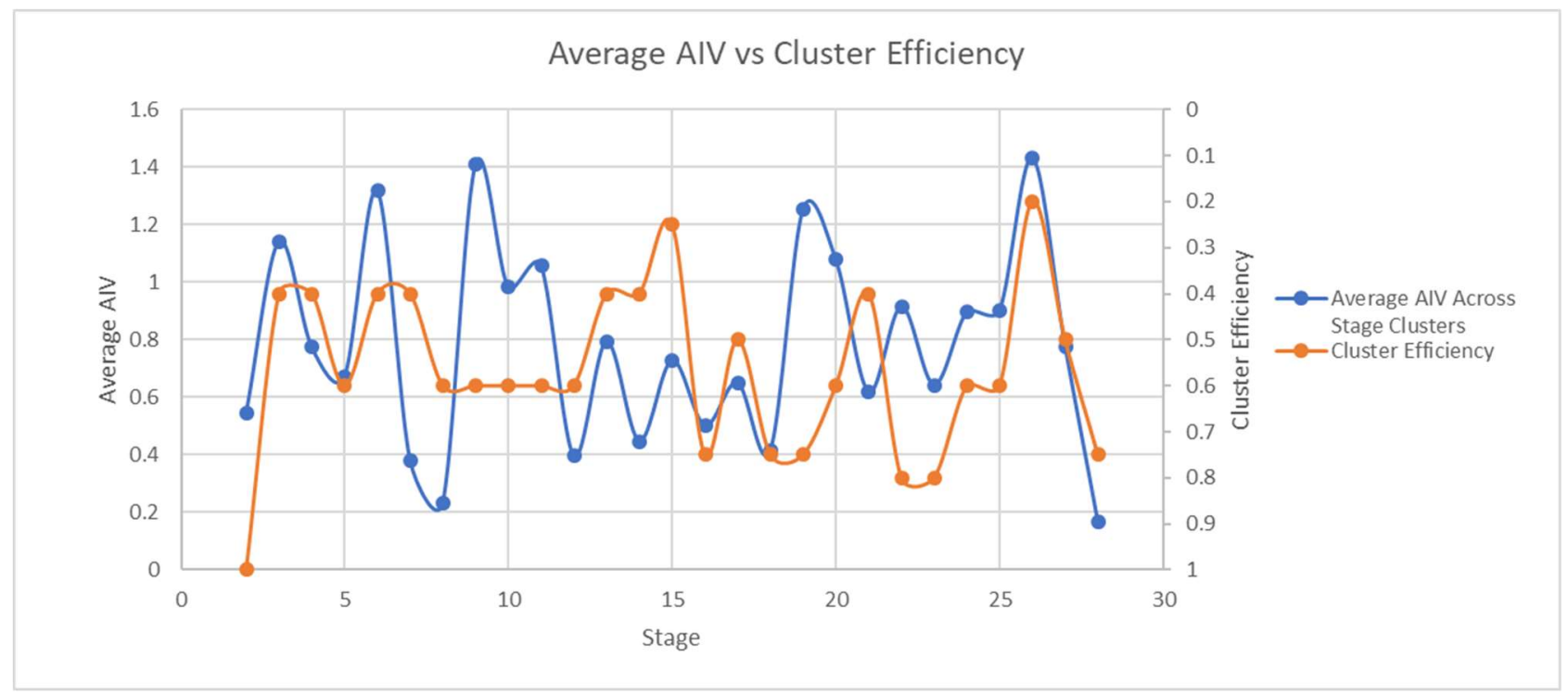

Figure 23 - Average AIV vs. Cluster Efficiency

\subsection{Using C-Means Fuzzy Clustering to Assist in Completions Design}

Since C-Means Fuzzy Clustering has shown that it is effective at characterizing shale likeness using well logs by comparing the classifications to per-cluster production, C-Means Fuzzy Clustering is believed to be able to work at a larger scale. This larger scale would be to classify the shales along the entire sonic scanner and natural fracture logs per half foot for the entire horizontal wellbore. This data could then be used to design well completions.

Using the same number of centroids as in the per cluster study (3), the full sonic scanner and natural fracture logs were passed to the C-Means Fuzzy Clustering algorithm. The result was a three-class fuzzy membership per each half foot along the entire horizontal wellbore for MIP-3H. With so many datapoints, the granularity seen in the study conducted in Section 4.3 is expected to be reduced. However, meaningful results are still attainable in that the algorithm can still detect significant changes in class membership and in doing so indicating anisotropy. 
This reduced granularity can be shown by again plotting the fuzzy partition coeffect (FPC) vs number of clusters as in Figure 24.

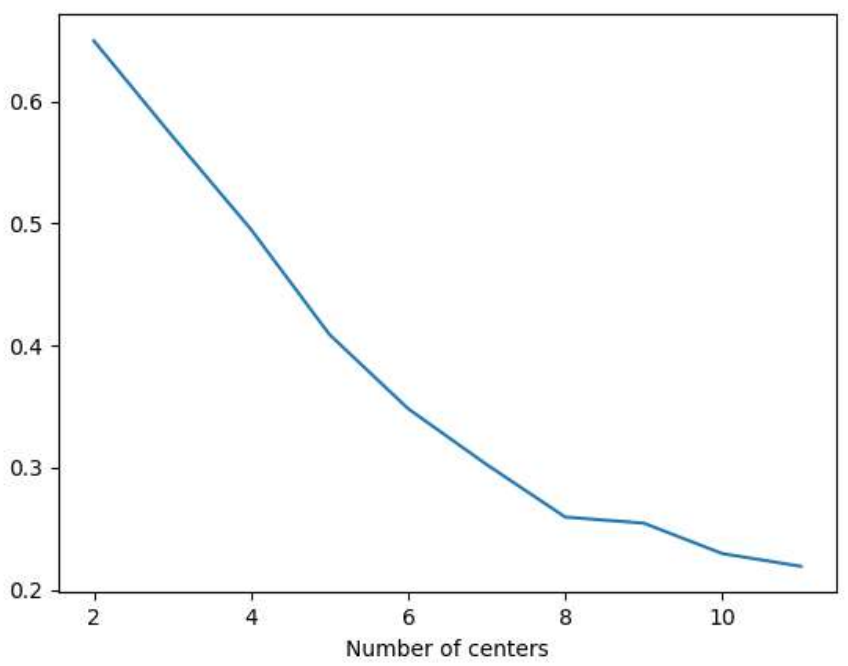

Figure 24 - FPC vs Number of Centers for Full Wellbore Clustering

Since the number of chosen clusters will remain three as it was in Section 4.3, you can see the FPC value decreased to 0.57 for 3 clusters. This is still above the minimum acceptable value of 0.5 , which is required to attain meaningful clustering results.

In order to interpret the results of the C-Means Fuzzy Clustering for the entire wellbore, two values need to be determined. First, what was the dominant class membership at each half foot? This was simply done by selecting the class with the highest degree of membership. Plotting the dominant class vs measured depth makes interpreting the plots on this scale significantly more manageable. The second action was to calculate the AIV at each half foot. Unlike the study in Section 4.3, the entire log can be interpreted continuously without having to take stages into account. This means that each data point at every half foot has an AIV 
calculated by relating that point's fuzzy membership to the point next to it on order to identify significant changes.

The initial portion of this study will focus on optimizing the cluster spacing using the fuzzy membership and the AIV and comparing this design to the one used in MIP $3 \mathrm{H}$. The number of clusters, and the stage lengths, which were implemented in the completions design of MIP $3 \mathrm{H}$ will remain constant. By plotting the dominant class membership vs, the MD for each stage, you can plot the locations of the MIP $3 \mathrm{H}$ clusters within the stage and you can plot the optimum cluster locations based on the AIV. The optimum location for the clusters is found by selecting an MD range where the dominant class membership is constant and selecting the point where the AIV is the lowest. These ranges were chosen to be 2 feet from any other dominant class as much as possible, since cluster perforations have an assigned total impact area of 2 feet.

Other guidelines that were deemed to be best practice and followed as much as possible are: a minimum cluster spacing of $40^{\prime}$ with a $15^{\prime}$ stand off from the beginning and end of each stage. As with in Section 4.3, Cluster 127 was not logged and therefore excluded from the study. Similarly, Stage 1 does not have reported cluster locations and is not included in the results in the Appendix. Figure 24 below shows the design for Stage 6, including the used cluster locations (red) and optimum cluster locations based on AIV (green). 


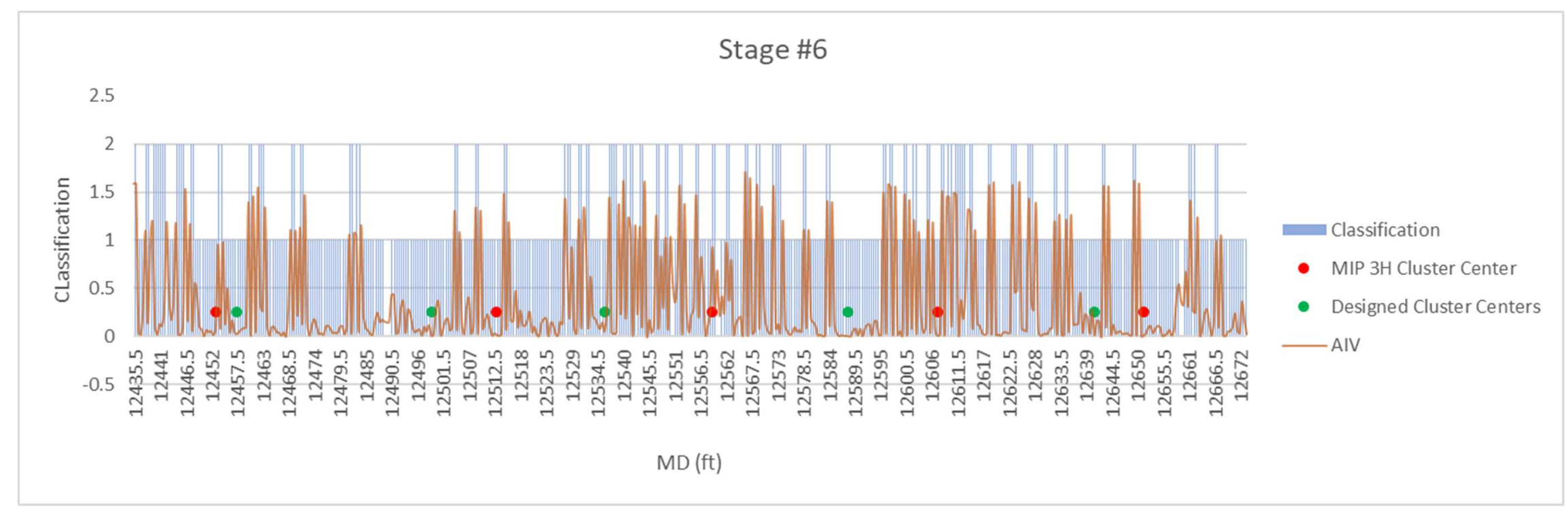

\begin{tabular}{|c|c|}
\hline Avg. AIV at MIP 3H Cluster Center & 0.5911 \\
\hline Avg. AIV & Censigen
\end{tabular}

\begin{tabular}{|l|l|}
\hline Avg. AIV at Designed Cluster Center & 0.01987 \\
\hline
\end{tabular}

Figure 25 - Optimum Cluster Design for Stage 6 
It can be observed that in Figure 25, the MIP-3H clusters 24 and 22 located at 12,453' and 12,559' respectively are within $+/-1$ feet of a dominant Class 2 area. These are the only two clusters that produced gas, since clusters 23,21 , and 20 located at 12,512', 12606', and 12650' respectively all produced zero gas. This is in line with what was reported in Figure 18 in Section 4.3. Note, the specific class definitions are different than in Figure 18. This is because every time data is passed through the C-Means algorithm, it names the centroids as class 0,1 or 2 at random. However, the fuzzy membership values remain constant as the names of the class change. As such, Class 2 in Figure 25 is representative of Class 0 in Figure 19.

When applying this optimum cluster design across the entire horizontal wellbore, these patterns with the results displayed in Section 4.3 are consistent, but not always exact. This is in part due to using a significantly larger dataset, with over 12,000 points of data compared to the dataset of 126 data points used in Section 4.3. However, being able to notice these patterns is even more encouraging that using C-Means Fuzzy Clustering to assist in completions design can be effective. As in Section 4.3, Figure 26 will next display the optimum cluster design results for Stage 13. 


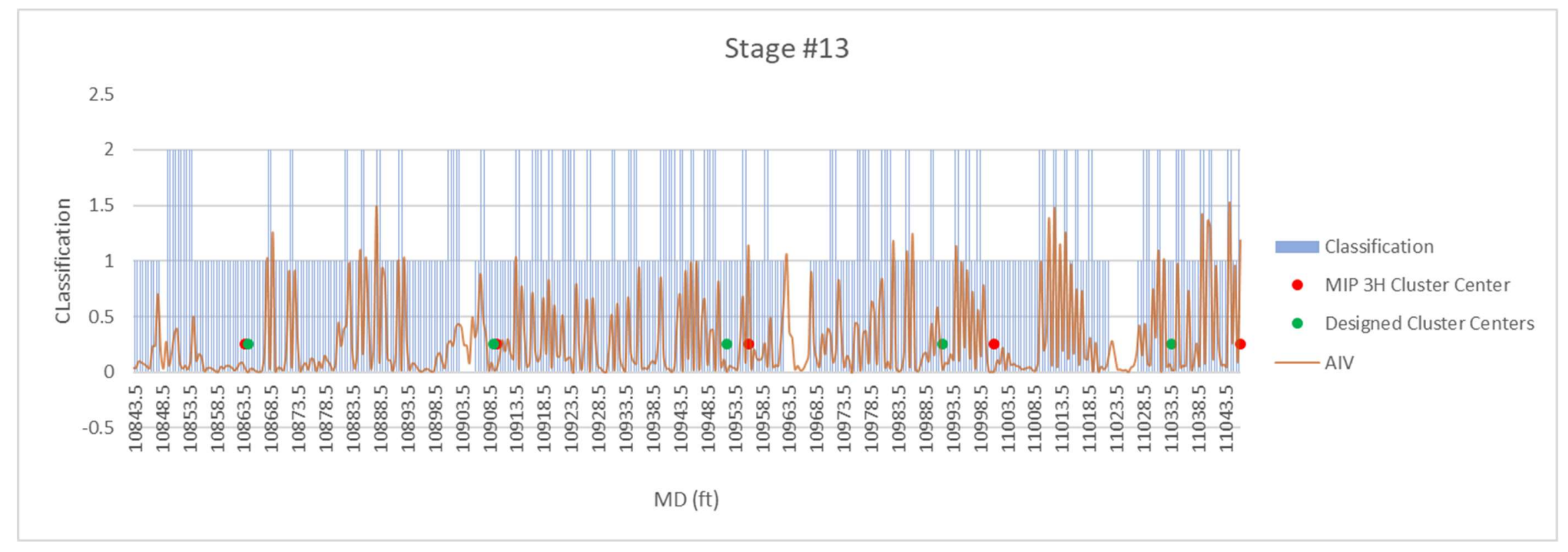

\begin{tabular}{|c|c|}
\hline Avg. AIV at MIP 3H Cluster Center & 0.36477 \\
\hline Avg. AIV at Designed Cluster Center & 0.01503 \\
\hline
\end{tabular}

Figure 26 - Optimum Cluster Design for Stage 13 
With stage 13, it can be observed that clusters 59 and 58, located at 10,836' and 10,909 ' respectively, were the only two clusters to produce gas. They were located squarely in Class 1 areas, with more than 2 feet on either side away from another class. The optimization using the AIV, placed the optimum clusters in very similar positions. Then nonproducing clusters of 57 and 55, located at 10956' and 11046' produced no gas and were located within a Class 2 areas +/- $1^{\prime}$ from their centers. Cluster 56 was located in a Class 1 area but was found to not be optimal using the AIV. The trend of Clusters 59, 58, and 56 having the same dominant class membership is identical to Figure 21, within Section 4.3. Using the AIV to optimize the cluster locations for stage 13 found significantly different locations than what was used for the non-producing clusters of 57,56 , and 55 . The cluster optimization plots for the 25 other available stages can be found in the Appendix under "MIP-3H Cluster Re-Design using CMeans".

The idea of using C-Means Fuzzy Clustering to optimize completions design can take a step further than simply using the fuzzy membership and AIV to place clusters in a stage. It can also be used to select the length of a stage. By plotting the fuzzy membership per half foot across the entire wellbore, patterns can be observed where there are consistent areas of a dominant class. It would be beneficial to group these areas of like lithology together within a specific stage in order to achieve an optimum number of clusters and cluster locations. This would avoid situations where one half of a stage is one dominant class and the other half of the stage is another dominant class. This effect would make it nearly impossible to evenly space clusters in an optimum way within a stage for one dominant class. The effect stages being half one dominant class and half another was observed in several stages for MIP-3H and could be 
avoiding by using the C-Means Fuzzy Clustering results to find the optimum number of stages, variable stage lengths, number of clusters, and cluster locations. The dominant class membership for the entire wellbore was plotted, divided into the optimum number of stages and plotted against the AIV. This Plot can be found in the Appendix under "Full MIP-3H Stage Design".

The result of selecting the stage lengths by grouping sections of the wellbore based on their dominant class was a new 23 stage design for MIP-3H. The design took into account some of the following constraints: there was a minimum of 40' spacing between clusters, a 15' minimum stand off from the beginning and end of each stage, the minimum allowable stage length was $150^{\prime}$, and the maximum stage length was $350^{\prime}$. The number of clusters designed per stage varied based on the stage length. If the length was between $150^{\prime}$ and $225^{\prime}$, the stage was designed with 4 clusters. If the stage was between $225^{\prime}$ and $300^{\prime}$, the stage was designed with 5 clusters. If the stage was between 300' and 350', the stage was designed with 6 clusters.

Overall, by varying the stage lengths to best fit an area of like rock based on its CMeans Fuzzy Clustering classification, it was significantly easier to recognize windows to optimize cluster placement by finding the minimum AIV in that range. For example, only one section of the wellbore was predominantly Class 0 . When considering the stage lengths used to complete MIP-3H, this section of Class 0 shales was split between two stages, Stage 10 and Stage 11 (these plots can be located in the Appendix). Since half of each stage was Class Zero dominant, it was difficult to optimize the cluster placement within the same lithological class. However, when considering the dominant class of lithology to design the stage length, it was 
possible to encapsulate the entire section of the MIP-3H wellbore that is Class 0 into one stage.

This was Designed Stage 9, as presented in Figure 27. 


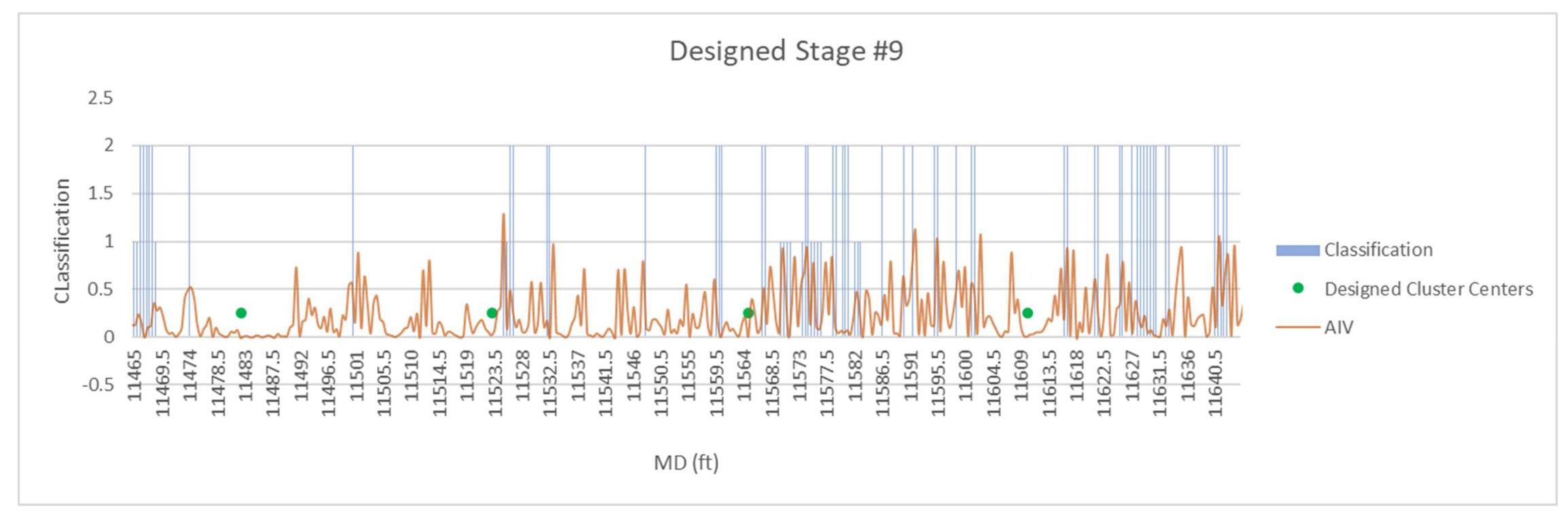

\section{\begin{tabular}{|l|l|}
\hline Avg. AIV at Designed Cluster Center & 0.0145 \\
\hline
\end{tabular}}

Figure 27 - Designed Stage \#9 
Being able to have a stage with such uniform lithology as presented in Designed Stage 9 could see an increase in fracture formation on a per cluster basis from what was seen in Stages 10 and 11 for MIP $3 \mathrm{H}$. these Stages only saw a $60 \%$ cluster efficiency, where the $40 \%$ of clusters that did not produce gas or propagate fractures were located in areas of different lithologic classes. By designing the stage to include as much of the same dominant lithologic class as possible, it becomes easier to assign cluster locations based on an optimum AIV. This was not just the case with Designed Stage \#9, but also along the entire wellbore. It was possible to isolate areas of dominant Class 1 and 2 shales as well, which could see improved production with optimum cluster placement. An example of a stage designed based on a dominant Class 1 lithology is shown in Figure 28 as Designed Stage \#12. 


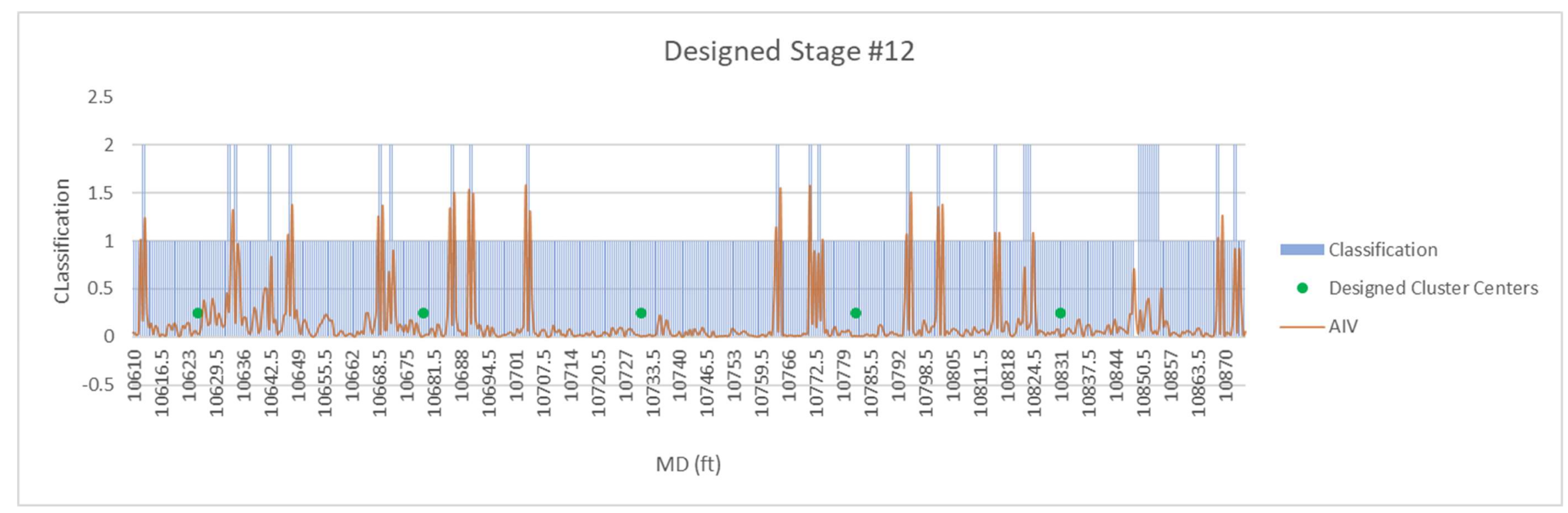

\begin{tabular}{|l|l|}
\hline Avg. AIV at Designed Cluster Center & 0.0074 \\
\hline
\end{tabular}

Figure 28 - Designed Stage \#12 
It can be observed with the Designed Stages 9 and 12 that it becomes efficient to place clusters if the dominant lithology is best represented within a single stage based on the C-Means Fuzzy Clustering of the logged data. The completions design parameters will certainly vary by company, but what was presented in this section is a promising initial step based on theory as well as the results presented by the MIP-3H case study. 


\section{Chapter 6: Conclusions}

Based on the results presented in Chapter 4, it can be observed that using C-Means Fuzzy Clustering on well logging data can yield important results to help improve the understanding behind the performance of unconventional shale wells. The first application was to use C-Means Fuzzy Clustering to detect shale anisotropy in the MIP-3H well and compare those results to the production log for MIP-3H to detect any correlation between the shale anisotropy and heterogeneity to the MIP-3H clusters with zero production. The magnitude of the shale anisotropy and heterogeneity was quantified using the variable anisotropy indicator value (AIV), which measured the change in C-Means classification at the measured depths relative to each cluster.

For MIP-3H, there were 126 available clusters to study based on the sonic scanner, natural fracture, and production logs. Of the 126 clusters, 56 produced zero gas based on the results of the production log. After preforming C-Means Fuzzy Clustering on the sonic scanner and natural fracture logs for MIP-3H, fuzzy classifications were applied to each cluster and were related using the AIV to detect a significant change in shale properties. It was determined that 49 of the 56 clusters producing zero gas occurred where there was high AIV values. This draws the conclusion that the shale anisotropy and heterogeneity are having an impact on certain clusters propagating successfully and producing gas within a stage, while other clusters are not. This is explained by these results, where certain areas of the shale within a stage are favorable for formation breakdown and will therefore take the majority of the energy induced during the frac, leaving the other less favorable formation to fail to propagate successfully. 
A potential source of error within this study leading to not having a $100 \%$ efficiency when detecting a high AIV at non-producing clusters using C-Means, is the depth of investigation of the geomechanical and natural fracture logging tools. The error comes from when the reservoir conditions vary significantly beyond the depth of investigation measured by the logging tool. Additionally, the conditions of the wellbore can be altered by drilling mud invasion and mud cake formation. Based on the results of the study which found a high percentage of nonproductive clusters occurring at areas of high AIV, the uncertainty associated with the logging tools can be considered impactful, but not detrimental to attaining meaningful results.

After observing $88 \%$ of zero-producing clusters occur at areas with a high AIV value, using C-Means Fuzzy Clustering to interpret the entire wellbore is the logical next step for this study. The initial objective was to be able to effectively classify the entire wellbore, and place all of the clusters on the same class of shales at points which have the minimum AIV. The expected outcome of such a design is that the shale rock properties at all of the clusters will be similar within a stage, and none of the clusters would propagate easier than the others thus consuming all of the hydraulic to mechanical energy of the frac.

The entire wellbore was classified using C-Means Fuzzy Clustering on the sonic scanner and natural logs. The first design was to use the same stage lengths as MIP-3H, and instead replace the cluster locations using the minimum AIV within areas of a single dominant class. The result was every cluster for all 27 stages was placed at locations of uniform class where the AIV is minimum. These results were compared to the actual MIP-3H cluster design, which again 
showed that often where there was a zero productive cluster, it was located on a different class at some point within the $2 \mathrm{ft}$ interval for that cluster.

However, it was observed that certain stages for MIP-3H would have stages where half of the stage would be one dominant class, and the other half would be a different dominant class. This made it difficult to effectively place clusters for the whole stage based on one dominant class with a minimum AIV. The solution was to use the C-Means Fuzzy Clustering results to design the stage lengths and cluster locations simultaneously. By using reasonable stage length and cluster spacing constraints, it was possible to redesign the entire completions based on the results of the C-Means Fuzzy Clustering. With this new completion design, each stage attempts to maximize the footage of one dominant class in order to more easily place the clusters in areas of minimum AIV for that class. This eliminated stages of mixed dominant classes and improved the ease, and likely the effectiveness, of the MIP-3H completions design.

In conclusion, operators could improve the efficiency of their unconventional well production by running sonic scanner and natural fracture logs on wells in order use C-Means Fuzzy Clustering to characterize the wellbore. Completions can then be designed based these characterizations with the goal of increasing the number of successful fractures per stage. The additional cost per well of running these logs could quickly be offset by the additional revenue gained from increasing production with an optimized per well completions design. 


\section{Chapter 7: Recommendations for Future Work}

There are several ways in which this project can be improved upon and further validated. First, the project can be reproduced on other wells where they have at least a sonic scanner log (or other geomechanical logs), fracture logs and a production log. The MSEEL project which supplied the data used in this study for well MIP-3H, has finished the drilling and completions of a second pad. This pad is called the MSEEL Boggess pad and is expected to produce very similar data to that used for MIP-3H. Replacing this project with data from those wells could be advantageous in continuing the proof of concept with further validation. Alternatively, data can be sourced from operators or other public databases in order to replicate this study.

Another potential future work is to develop a digital tool that simplifies this process for the user, and potentially using an Al and Machine Learning algorithm to design an entire wells completion by simply passing it the logging data. Such an algorithm would incorporate the classifications from the C-Means Fuzzy Clustering and the AIV to optimize the stage lengths and

cluster locations instantaneously. Such a tool could be used in industry to design the completions for a pad quickly without using a geometric design, while also promoting the potential for improved production.

Another way to improve this line of work is to expand the focus beyond clusters that produce zero gas. There were many clusters on the MIP-3H lateral that produce a very small amount of gas, between 0 and $20 \mathrm{mcfd}$. The study can be expanded to include an analysis of these clusters, and how they relate to how the completions were conducted (i.e. pump rate, 
breakdown pressure, proppant side, etc). By normalizing the per cluster production to some of the data from the completions, trends may emerge on how those completions parameters are impacting the production. This could be especially prevalent with MSEEL wells like MIP-3H and the future Boggess wells because some stages are used to test different methods and products. It could be pertinent to try to normalize the production based on what was done during the completions. This study can also be improved upon by considering other reservoir and fluid flow properties such as porosity, permeability, and total organic content (TOC). This study ignored their variations along the wellbore, and they can be incorporated in the future to better understand the well's productivity.

Future field lab studies can also be conducting using the proposed technique in order to validate the outcomes and test whether there will be improved production efficiency. This would best be done by collaborating with a well operating company to attain a sample size of wells and compare the production from the wells using the completions optimization strategy to wells with standard geometric designs. Such a study could serve to change the way unconventional wells are completed, in order to avoid clusters with zero contribution to well production.

The idea of using Al and Machine Learning to optimize unconventional well completions can also be applied to many other projects in the oil and gas industry, such as optimizing drilling operations. By classifying sets of lithological geomechanical data, optimum drilling designs for unconventional wells could be discovered and predicted. Likewise, drilling parameters such as weight-on-bit, RPMs, torque, bit type, etc. could be optimized using C-Means Fuzzy Clustering 
in order to identify drilling methods that produce the most effective results in different formations. 


\section{References}

[1]. Gerdom, Dewey, et al. "Geomechanics Key In Marcellus Wells." The American Oil and Gas Reporter, Schlumberger, Mar. 2013, www.slb.com/-/media/files/stimulation/industryarticle/201303-og-geomechanics.

[2]. Cook, Troy, and Jack Perrin. "Hydraulic Fracturing Accounts for about Half of Current U.S. Crude Oil Production." U.S. Energy Information Administration, 15 Mar. 2016, www.eia.gov/todayinenergy/detail.php?id=25372.

[3]. “ 4-Week Avg U.S. Net Imports of Crude Oil and Petroleum Products ." U.S. Energy Information Administration, 21 Oct. 2020, www.eia.gov/dnav/pet/hist/LeafHandler.ashx?n=pet\&s=wttntus2\&f=4.

[4]. "10 Biggest Shale Plays in the US.” Worldwide Power Products, 2020, www.wpowerproducts.com/news/10-biggest-shale-plays-in-the-us-revised/.

[5]. Smythe, David. "Faulting in the US Shale Basins." DavidSmythe.org, 17 July 2017, www.davidsmythe.org/fracking/USfaulting.htm.

[6]. Shepstone, Tom. "FERC Report Tells the Story as the Shale Revolution Moves Ever Forward." Shale Directories, 2018, www.shaledirectories.com/blog/ferc-report-tells-thestory-as-the-shale-revolution-moves-ever-forward/.

[7]. Popova, Olga. "Marcellus Shale Play - Geology Review." U.S. Energy Information Administration, 2017, www.eia.gov/maps/pdf/MarcellusPlayUpdate_Jan2017.pdf.

[8]. Milici, Robert, and Christopher Swezey. "Assessment of Appalachian Basin Oil and Gas Resources: Devonian Shale-Middle and Upper Paleozoic Total Petroleum System." United States Geological Survey, U. S. Department of the Interior, 2006, pubs.usgs.gov/of/2006/1237/of2006-1237.pdf.

[9]. Barth, James O, et al. "Frac Diagnostics Key In Marcellus Wells." The American Oil and Gas Reporter, May 2012, www.aogr.com/magazine/frac-facts/frac-diagnostics-key-inmarcellus-wells.

[10]. White, Kathleen Hartnett. "The Fracas about Fracking - Low Risk, High Reward -- but the EPA Is against It.” Energy In Depth, National Review Online, June 2011, www.energyindepth.org/wp-content/uploads/2011/06/The-Fracas-about-Fracking.pdf.

[11]. Pearson, C Mark. "Hydraulic Fracturing of Horizontal Wells - Realizing the Paradigm Shift That Has Been 30 Years in Development ." Liberty Resources LLC., Society of Petroleum Engineers, 2020, libertyresourcesllc.com/upload/SPE\%20Distinguished\%20Lecture\%20$\% 20$ Horizontal\%20Well\%20Fracturing.pdf. 
[12]. Beard, Tim. "EPA Hydraulic Fracturing Workshop." U.S. Energy Information Administration, Chesapeake Energy Corporation, Mar. 2011, www.epa.gov/sites/production/files/documents/fracturedesigninhorizontalshalewells.pdf.

[13]. Ma, Zee, et al. "Integrated Shale Gas Reservoir Modeling." Research Gate, Dec. 2011, www.researchgate.net/figure/Horizontal-well-completion-example-2000-ft-longhorizontal-section-4-stages-of_fig6_270340648.

[14]. “Surface \& Downhole Logging." Schlumberger, 2020, www.slb.com/reservoircharacterization/surface-and-downhole-logging.

[15]. "Wireline Openhole Logging." Schlumberger, 2020, www.slb.com/reservoircharacterization/surface-and-downhole-logging/wireline-openhole-logging.

[16]. “ Sonic Scanner.” Schlumberger, 2020, www.slb.com/reservoir-characterization/surfaceand-downhole-logging/wireline-cased-hole-logging/sonic-scanner-platform.

[17]. Lafone, Frank. “ MIP Well Datasets .” Marcellus Shale Energy and Environmental Laboratory, 2015, mseel.org/research/research_MIP.html.

[18]. Wang, HanYi. "Hydraulic Fracture Propagation in Naturally Fractured Reservoirs: Complex Fracture or Fracture Networks." Science Direct, 2019, www.sciencedirect.com/science/article/abs/pii/S1875510019301556.

[19]. "MicroScope HD.” Schlumberger, 2017, www.slb.com//media/files/drilling/brochure/microscope-hd-br.ashx.

[20]. Dundar, Emre Can, Alhemdi A, Gu M. "Impact of Natural Fracture Induced Elastic Anisotropy on Completion and Fracturing Design in Different Shale Reservoirs." One Petro, Unconventional Resources Technology Conference, July 2019, www-onepetroorg.www.libproxy.wvu.edu/download/conference-paper/URTEC-2019-893MS?id=conference-paper\%2FURTEC-2019-893-MS.

[21]. Salah, Mohamed, et al. "Influences of Anisotropy on Shale Brittleness Evaluation." One Petro, Society of Petroleum Engineers, Oct. 2019, www-onepetroorg.www.libproxy.wvu.edu/download/conference-paper/SPE-195874-MS?id=conferencepaper\%2FSPE-195874-MS.

[22]. Vishkai, M, et al. "Influence of Stress Anisotropy on Hydraulic Fracturing." One Petro, American Rock Mechanics Association, June 2014, www-onepetroorg.www.libproxy.wvu.edu/download/conference-paper/ARMA-20147360?id=conference-paper\%2FARMA-2014-7360.

[23]. McGinley, Mark, et al. "The Effects of Fracture Orientation and Elastic Property Anisotropy OnHydraulic Fracture Conductivity in the Marcellus Shale." One Petro, Society of Petroleum Engineers, Sept. 2015, www-onepetro- 
org.www.libproxy.wvu.edu/download/conference-paper/SPE-174870-MS?id=conferencepaper\%2FSPE-174870-MS.

[24]. Market, Jennifer, et al. "Untangling Acoustic Anisotropy." One Petro, Oct. 2015, wwwonepetro-org.www.libproxy.wvu.edu/download/journal-paper/SPWLA-2015v56n5a1?id=journal-paper\%2FSPWLA-2015-v56n5a1.

[25]. Esmersoy, Cengiz, et al. "Dipole Shear Anisotropy Logging." One Petro, www-onepetroorg.www.libproxy.wvu.edu/download/conference-paper/SEG-1994-1139?id=conferencepaper\%2FSEG-1994-1139.

[26]. Krasnikov, Artem, et al. "Consideration of Elastic Properties and Stresses Anisotropy in Fracturing Planning." One Petro, Society of Petroleum Engineers, Oct. 2019, wwwonepetro-org.www.libproxy.wvu.edu/download/conference-paper/SPE-196899MS?id=conference-paper\%2FSPE-196899-MS.

[27]. Kahn, Safdar, et al. "Impact of Mechanical Anisotropy on Design of Hydraulic Fracturing in Shales." One Petro, Society of Petroleum Engineers, Nov. 2012, www-onepetroorg.www.libproxy.wvu.edu/download/conference-paper/SPE-162138-MS?id=conferencepaper\%2FSPE-162138-MS.

[28]. Hadi, Keivani, et al. "Experimental Study on Hydraulic Fracturing of Homogenous and Nonhomogenous Rock Samples." Research Gate, Apr. 2018, www.researchgate.net/publication/324605344_Experimental_study_on_hydraulic_fracturi ng_of_homogenous_and_non-homogenous_rock_samples.

[29]. Marr, Bernard. "27 Incredible Examples Of AI And Machine Learning In Practice." Forbes, 30 Apr. 2018, www.forbes.com/sites/bernardmarr/2018/04/30/27-incredibleexamples-of-ai-and-machine-learning-in-practice/\#def34fd75022.

[30]. Mohaghegh, Shahab D. "Shale Analytics Part 3: Artificial Intelligence \& Machine Learning." ECampus, West Virginia University, 2020.

[31]. Thompson, Wayne, et al. "Artificial Intelligence, Machine Learning, Deep Learning and Beyond." SAS, 2020, www.sas.com/en_us/insights/articles/big-data/artificial-intelligencemachine-learning-deep-learning-and-beyond.html.

[32]. Elisa. "What Is Fuzzy Logic?" CalTech, 28 Sept. 2006, www.cds.caltech.edu/ murray/wiki/index.php/What_is_fuzzy_logic\%3F.

[33]. Mohaghegh, Shahab D. Shale Analytics Data-Driven Analytics in Unconventional Resources. Springer, 2017.

[34]. "Fuzzy C-Means Clustering.” A Tutorial on Clustering Algorithms, 2020, matteucci.faculty.polimi.it/Clustering/tutorial_html/cmeans.html. 
[35]. Carr, Tim. "Marcellus Shale Energy and Environment Laboratory." MSEEL, National Energy and Technology Laboratory, West Virginia University, Northeast Natural Energy, Schlumberger, 2015, www.mseel.org/.

[36]. "Fuzzy c-Means Clustering.” Scikit Fuzzy, Python Hosted, 2020, pythonhosted.org/scikitfuzzy/auto_examples/plot_cmeans.html. 


\section{Appendix}

MIP-3H Production Log

\begin{tabular}{|c|c|c|c|c|c|c|}
\hline Bot & $\begin{array}{c}\text { Gas } \\
(1000 \\
\mathrm{ft} 3 / \mathrm{d})\end{array}$ & $\begin{array}{c}\text { Gas } \\
(\%)\end{array}$ & Stage & $\begin{array}{c}\text { Gas } \\
\text { per } \\
\text { Stage } \\
(\%)\end{array}$ & & Cluster \\
\hline 7755 & 17 & $0 \%$ & 28 & $1 \%$ & 0 & 127 \\
\hline 7805 & 17 & $0 \%$ & & & 0 & 126 \\
\hline 7854 & 0 & $0 \%$ & & & 1 & 125 \\
\hline 7903 & 17 & $0 \%$ & & & 0 & 124 \\
\hline 7946 & 45 & $1 \%$ & 27 & $2 \%$ & 0 & 123 \\
\hline 7995 & 39 & $1 \%$ & & & 0 & 122 \\
\hline 8044 & 0 & $0 \%$ & & & 1 & 121 \\
\hline 8087 & 0 & $0 \%$ & & & 1 & 120 \\
\hline 8130 & 67 & $1 \%$ & 26 & $1 \%$ & 0 & 119 \\
\hline 8177 & 0 & $0 \%$ & & & 1 & 118 \\
\hline 8222 & 0 & $0 \%$ & & & 1 & 117 \\
\hline 8266 & 0 & $0 \%$ & & & 1 & 116 \\
\hline 8312 & 0 & $0 \%$ & & & 1 & 115 \\
\hline 8358 & 59 & $1 \%$ & 25 & $3 \%$ & 0 & 114 \\
\hline 8402 & 0 & $0 \%$ & & & 1 & 113 \\
\hline 8450 & 0 & $0 \%$ & & & 1 & 112 \\
\hline 8497 & 38 & $1 \%$ & & & 0 & 111 \\
\hline 8543 & 62 & $1 \%$ & & & 0 & 110 \\
\hline 8589 & 60 & $1 \%$ & 24 & $4 \%$ & 0 & 109 \\
\hline 8636 & 0 & $0 \%$ & & & 1 & 108 \\
\hline 8680 & 67 & $1 \%$ & & & 0 & 107 \\
\hline 8726 & 0 & $0 \%$ & & & 1 & 106 \\
\hline 8765 & 65 & $1 \%$ & & & 0 & 105 \\
\hline 8816 & 63 & $1 \%$ & 23 & $5 \%$ & 0 & 104 \\
\hline 8865 & 72 & $1 \%$ & & & 0 & 103 \\
\hline 8912 & 0 & $0 \%$ & & & 1 & 102 \\
\hline 8961 & 53 & $1 \%$ & & & 0 & 101 \\
\hline 9007 & 84 & $2 \%$ & & & 0 & 100 \\
\hline 9053 & 82 & $2 \%$ & 22 & $7 \%$ & 0 & 99 \\
\hline 9089 & 82 & $2 \%$ & & & 0 & 98 \\
\hline 9138 & 0 & $0 \%$ & & & 1 & 97 \\
\hline 9185 & 76 & $1 \%$ & & & 0 & 96 \\
\hline 9229 & 149 & $3 \%$ & & & 0 & 95 \\
\hline 9273 & 165 & $3 \%$ & 21 & $4 \%$ & 0 & 94 \\
\hline 9320 & 34 & $1 \%$ & & & 0 & 93 \\
\hline 9367 & 0 & $0 \%$ & & & 1 & 92 \\
\hline 9412 & 0 & $0 \%$ & & & 1 & 91 \\
\hline 9447 & 0 & $0 \%$ & & & 1 & 90 \\
\hline 9506 & 35 & $1 \%$ & 20 & $2 \%$ & 0 & 89 \\
\hline 9554 & 0 & $0 \%$ & & & 1 & 88 \\
\hline
\end{tabular}




\begin{tabular}{|c|c|c|c|c|c|c|}
\hline 9596 & 0 & $0 \%$ & & & 1 & 87 \\
\hline 9641 & 33 & $1 \%$ & & & 0 & 86 \\
\hline 9687 & 31 & $1 \%$ & & & 0 & 85 \\
\hline 9734 & 91 & $2 \%$ & 19 & $2 \%$ & 0 & 84 \\
\hline 9781 & 14 & $0 \%$ & & & 0 & 83 \\
\hline 9825 & 14 & $0 \%$ & & & 0 & 82 \\
\hline 9871 & 0 & $0 \%$ & & & 1 & 81 \\
\hline 9914 & 91 & $2 \%$ & 18 & $4 \%$ & 0 & 80 \\
\hline 9961 & 74 & $1 \%$ & & & 0 & 79 \\
\hline 10004 & 66 & $1 \%$ & & & 0 & 78 \\
\hline 10050 & 0 & $0 \%$ & & & 1 & 77 \\
\hline 10092 & 105 & $2 \%$ & 17 & $5 \%$ & 0 & 76 \\
\hline 10094 & 0 & $0 \%$ & & & 1 & 75 \\
\hline 10136 & 145 & $3 \%$ & & & 0 & 74 \\
\hline 10141 & 0 & $0 \%$ & & & 1 & 73 \\
\hline 10280 & 185 & $3 \%$ & 16 & $7 \%$ & 0 & 72 \\
\hline 10318 & 142 & $3 \%$ & & & 0 & 71 \\
\hline 10367 & 0 & $0 \%$ & & & 1 & 70 \\
\hline 10410 & 50 & $1 \%$ & & & 0 & 69 \\
\hline 10458 & 59 & $1 \%$ & 15 & $1 \%$ & 0 & 68 \\
\hline 10505 & 0 & $0 \%$ & & & 1 & 67 \\
\hline 10549 & 0 & $0 \%$ & & & 1 & 66 \\
\hline 10596 & 0 & $0 \%$ & & & 1 & 65 \\
\hline 10641 & 107 & $2 \%$ & 14 & $4 \%$ & 0 & 64 \\
\hline 10685 & 96 & $2 \%$ & & & 0 & 63 \\
\hline 10733 & 0 & $0 \%$ & & & 1 & 62 \\
\hline 10776 & 0 & $0 \%$ & & & 1 & 61 \\
\hline 10824 & 0 & $0 \%$ & & & 1 & 60 \\
\hline 10865 & 185 & $3 \%$ & 13 & $5 \%$ & 0 & 59 \\
\hline 10911 & 84 & $2 \%$ & & & 0 & 58 \\
\hline 10957 & 0 & $0 \%$ & & & 1 & 57 \\
\hline 11002 & 0 & $0 \%$ & & & 1 & 56 \\
\hline 11048 & 0 & $0 \%$ & & & 1 & 55 \\
\hline 11095 & 69 & $1 \%$ & 12 & $4 \%$ & 0 & 54 \\
\hline 11142 & 69 & $1 \%$ & & & 0 & 53 \\
\hline 11189 & 104 & $2 \%$ & & & 0 & 52 \\
\hline 11232 & 0 & $0 \%$ & & & 1 & 51 \\
\hline 11279 & 0 & $0 \%$ & & & 1 & 50 \\
\hline 11326 & 170 & $3 \%$ & 11 & $6 \%$ & 0 & 49 \\
\hline 11373 & 111 & $2 \%$ & & & 0 & 48 \\
\hline 11418 & 35 & $1 \%$ & & & 0 & 47 \\
\hline 11459 & 0 & $0 \%$ & & & 1 & 46 \\
\hline 11511 & 0 & $0 \%$ & & & 1 & 45 \\
\hline 11559 & 25 & $0 \%$ & 10 & $4 \%$ & 0 & 44 \\
\hline 11603 & 83 & $2 \%$ & & & 0 & 43 \\
\hline 11651 & 82 & $2 \%$ & & & 0 & 42 \\
\hline 11697 & 0 & $0 \%$ & & & 1 & 41 \\
\hline 11737 & 0 & $0 \%$ & & & 1 & 40 \\
\hline
\end{tabular}




\begin{tabular}{|c|c|c|c|c|c|c|}
\hline 11784 & 77 & $1 \%$ & 9 & $3 \%$ & 0 & 39 \\
\hline 11830 & 0 & $0 \%$ & & & 1 & 38 \\
\hline 11882 & 74 & $1 \%$ & & & 0 & 37 \\
\hline 11927 & 17 & $0 \%$ & & & 0 & 36 \\
\hline 11973 & 0 & $0 \%$ & & & 1 & 35 \\
\hline 12017 & 0 & $0 \%$ & 8 & $3 \%$ & 1 & 34 \\
\hline 12060 & 84 & $2 \%$ & & & 0 & 33 \\
\hline 12100 & 54 & $1 \%$ & & & 0 & 32 \\
\hline 12145 & 0 & $0 \%$ & & & 1 & 31 \\
\hline 12196 & 15 & $0 \%$ & & & 0 & 30 \\
\hline 12241 & 85 & $2 \%$ & 7 & $3 \%$ & 0 & 29 \\
\hline 12287 & 0 & $0 \%$ & & & 1 & 28 \\
\hline 12327 & 57 & $1 \%$ & & & 0 & 27 \\
\hline 12369 & 0 & $0 \%$ & & & 1 & 26 \\
\hline 12420 & 0 & $0 \%$ & & & 1 & 25 \\
\hline 12454 & 115 & $2 \%$ & 6 & $5 \%$ & 0 & 24 \\
\hline 12514 & 0 & $0 \%$ & & & 1 & 23 \\
\hline 12560 & 142 & $3 \%$ & & & 0 & 22 \\
\hline 12608 & 0 & $0 \%$ & & & 1 & 21 \\
\hline 12652 & 0 & $0 \%$ & & & 1 & 20 \\
\hline 12699 & 0 & $0 \%$ & 5 & $6 \%$ & 1 & 19 \\
\hline 12748 & 47 & $1 \%$ & & & 0 & 18 \\
\hline 12795 & 63 & $1 \%$ & & & 0 & 17 \\
\hline 12839 & 200 & $4 \%$ & & & 0 & 16 \\
\hline 12886 & 0 & $0 \%$ & & & 1 & 15 \\
\hline 12934 & 31 & $1 \%$ & 4 & $1 \%$ & 0 & 14 \\
\hline 12979 & 0 & $0 \%$ & & & 1 & 13 \\
\hline 13027 & 44 & $1 \%$ & & & 0 & 12 \\
\hline 13070 & 0 & $0 \%$ & & & 1 & 11 \\
\hline 13120 & 0 & $0 \%$ & & & 1 & 10 \\
\hline 13164 & 0 & $0 \%$ & 3 & $1 \%$ & 1 & 9 \\
\hline 13214 & 22 & $0 \%$ & & & 0 & 8 \\
\hline 13261 & 0 & $0 \%$ & & & 1 & 7 \\
\hline 13307 & 36 & $1 \%$ & & & 0 & 6 \\
\hline 13355 & 0 & $0 \%$ & & & 1 & 5 \\
\hline 13400 & 131 & $2 \%$ & 2 & $5 \%$ & 0 & 4 \\
\hline 13445 & 84 & $2 \%$ & & & 0 & 3 \\
\hline 13492 & 21 & $0 \%$ & & & 0 & 2 \\
\hline 13534 & 21 & $0 \%$ & & & 0 & 1 \\
\hline 13811 & 251 & $5 \%$ & $2-1$ & $5 \%$ & 0 & 0 \\
\hline
\end{tabular}


Quality Analysis Plots for MIP-3H Logs
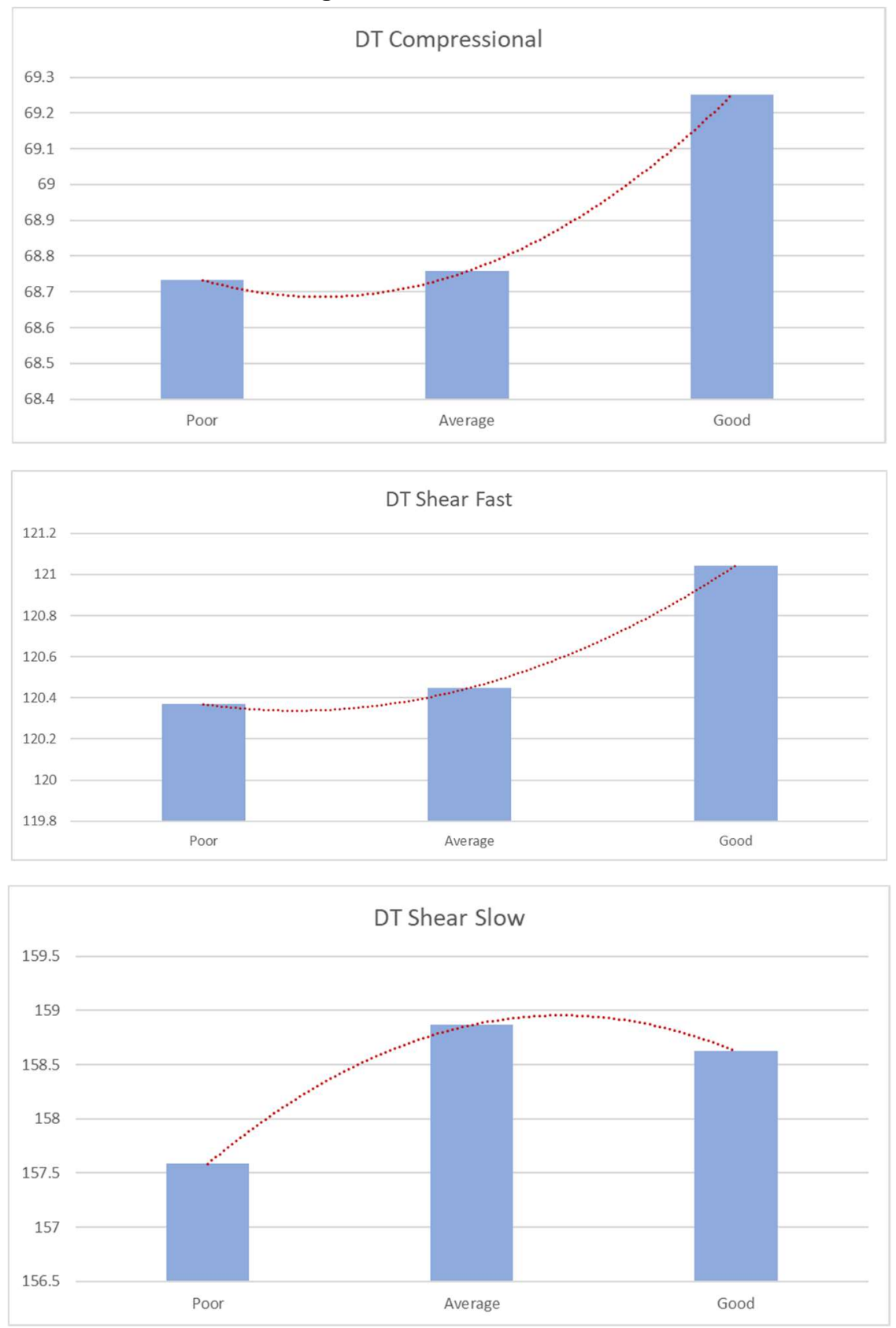

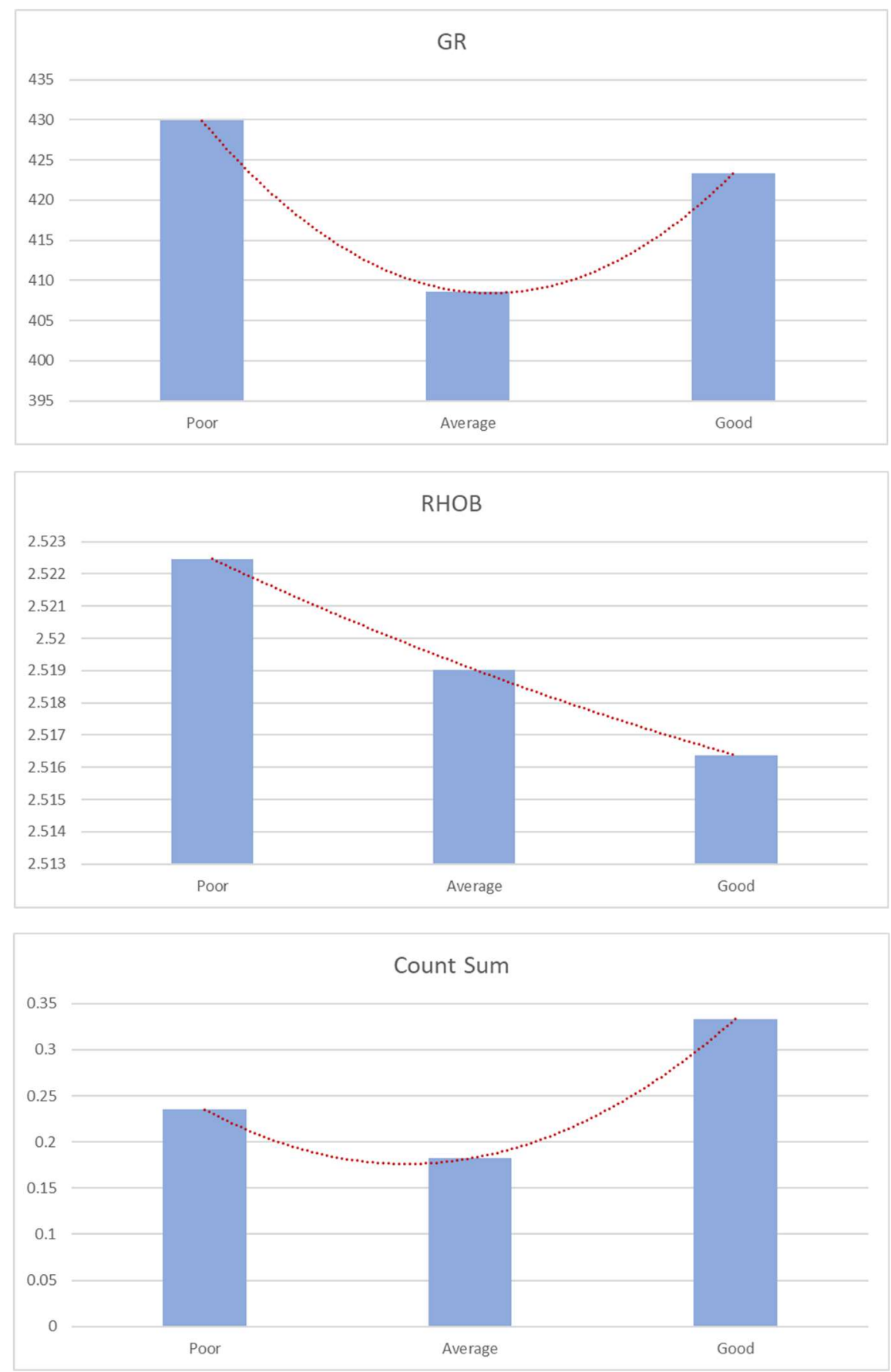

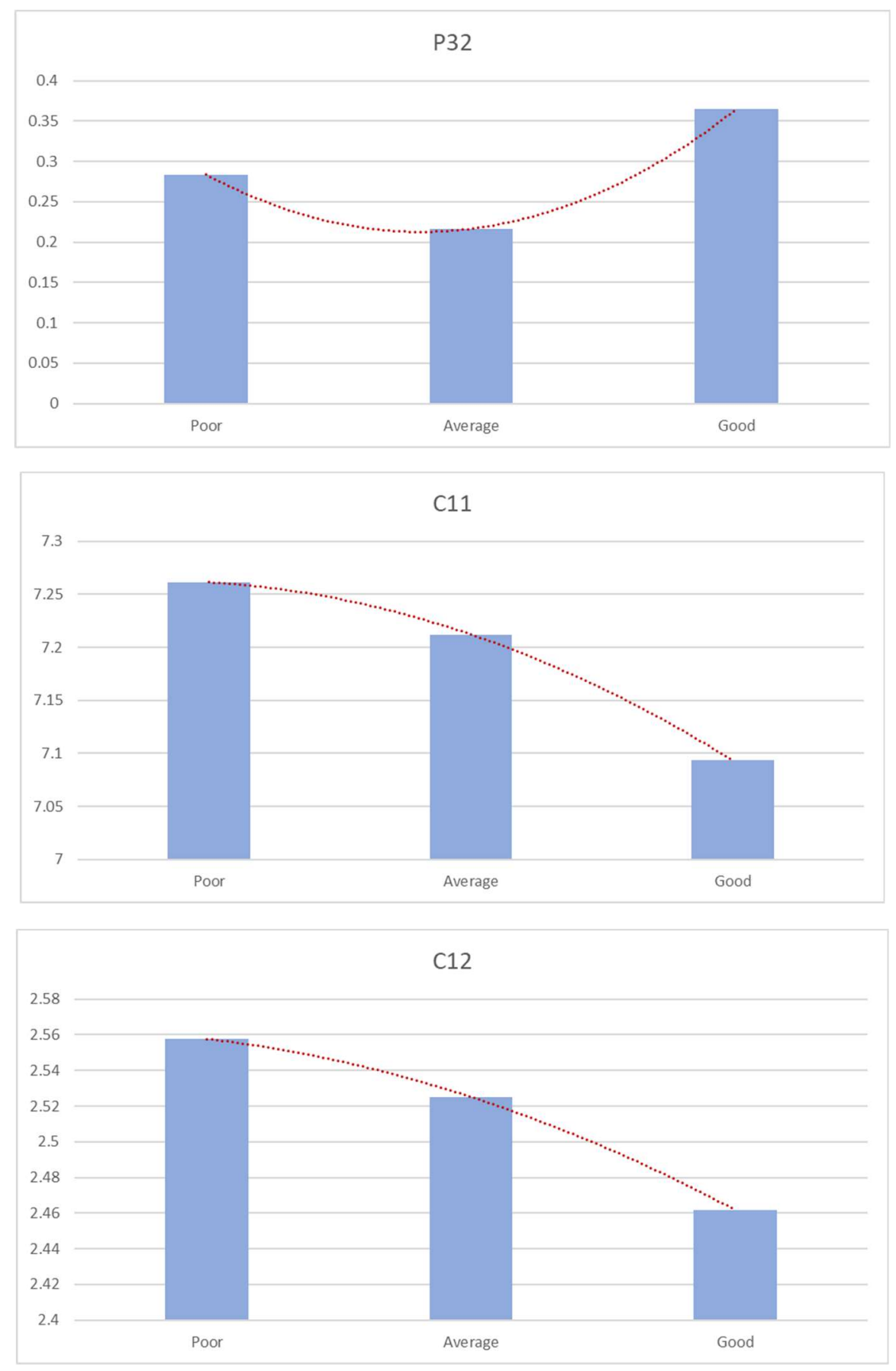

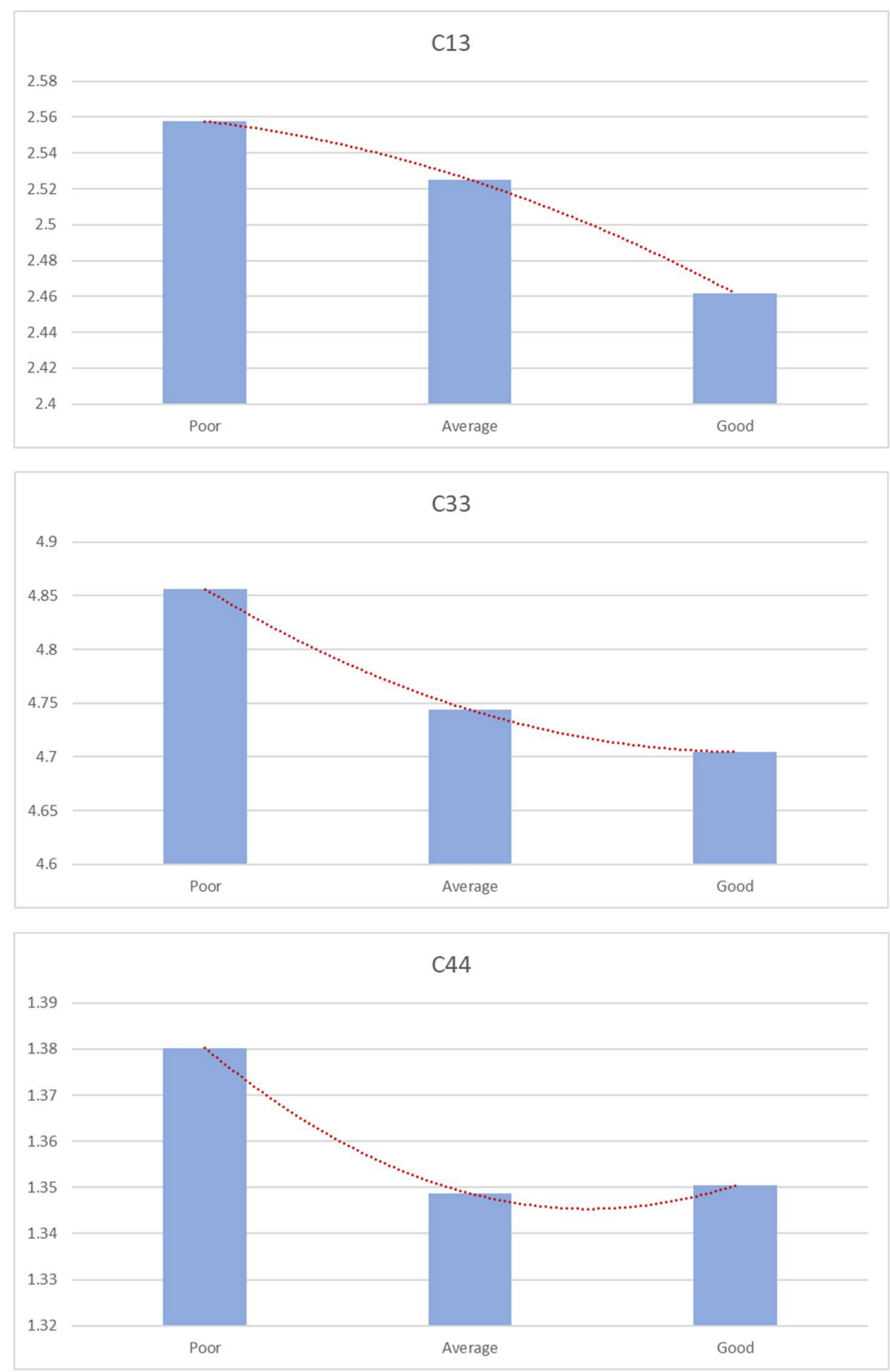

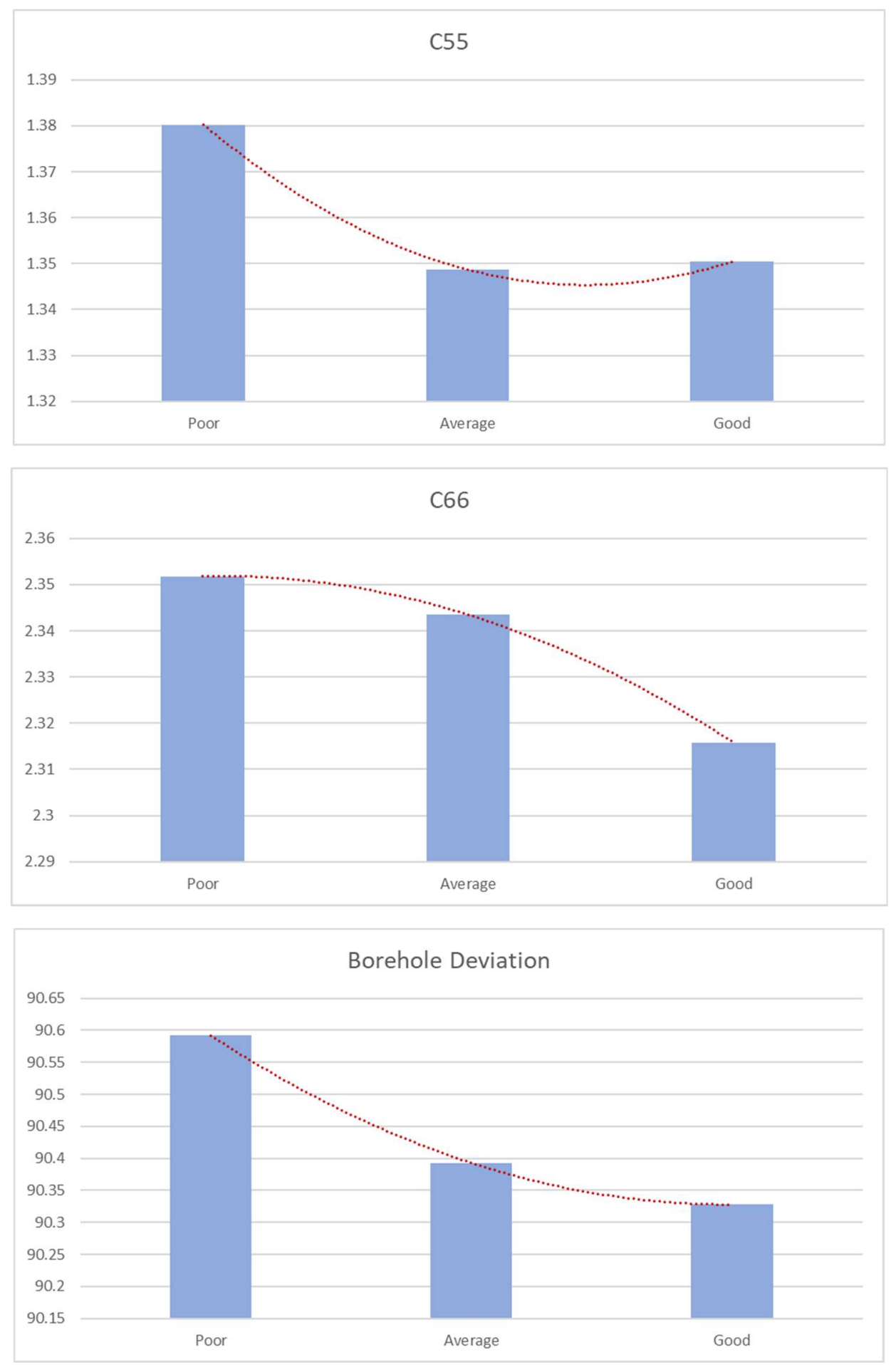

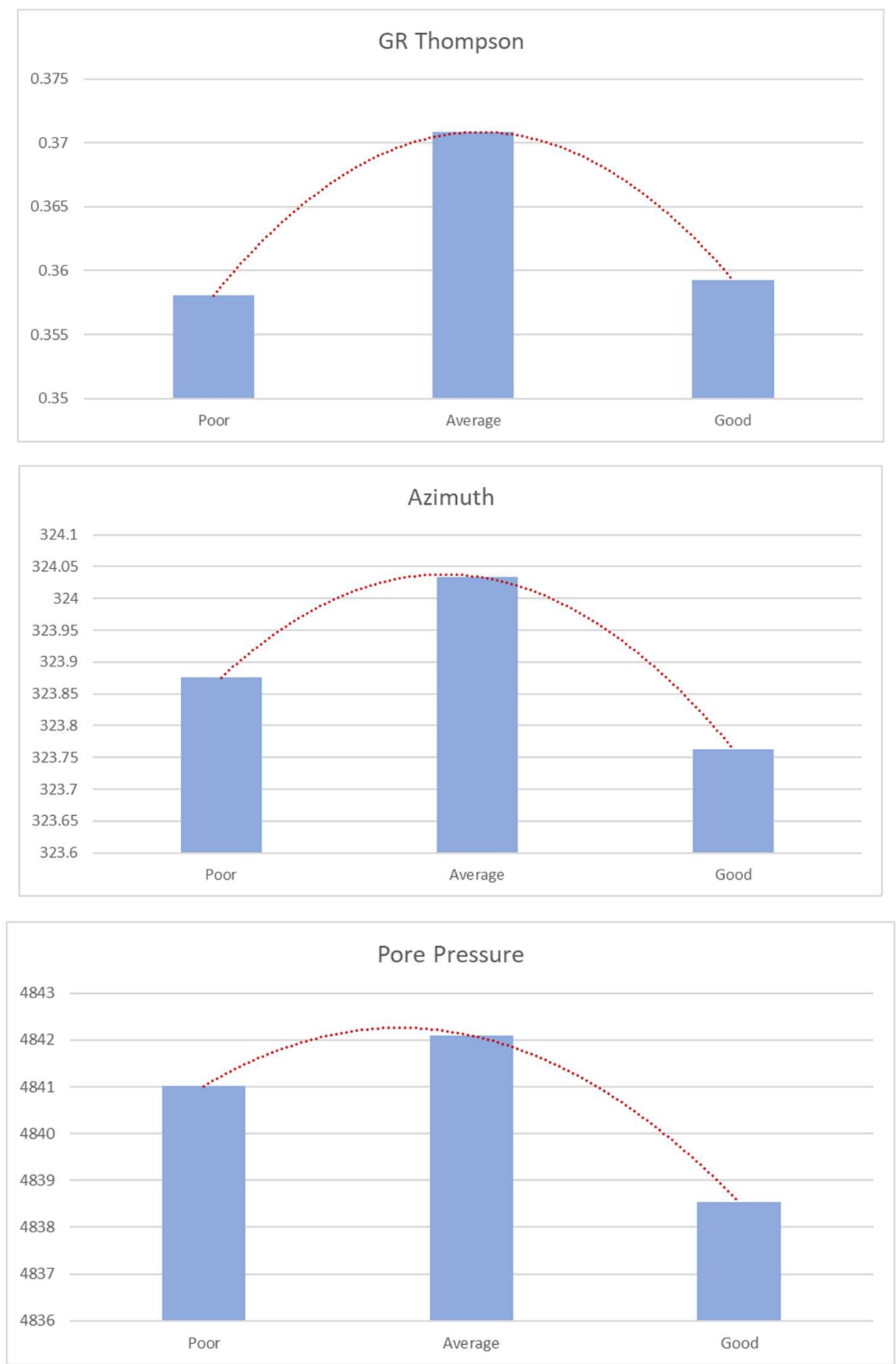

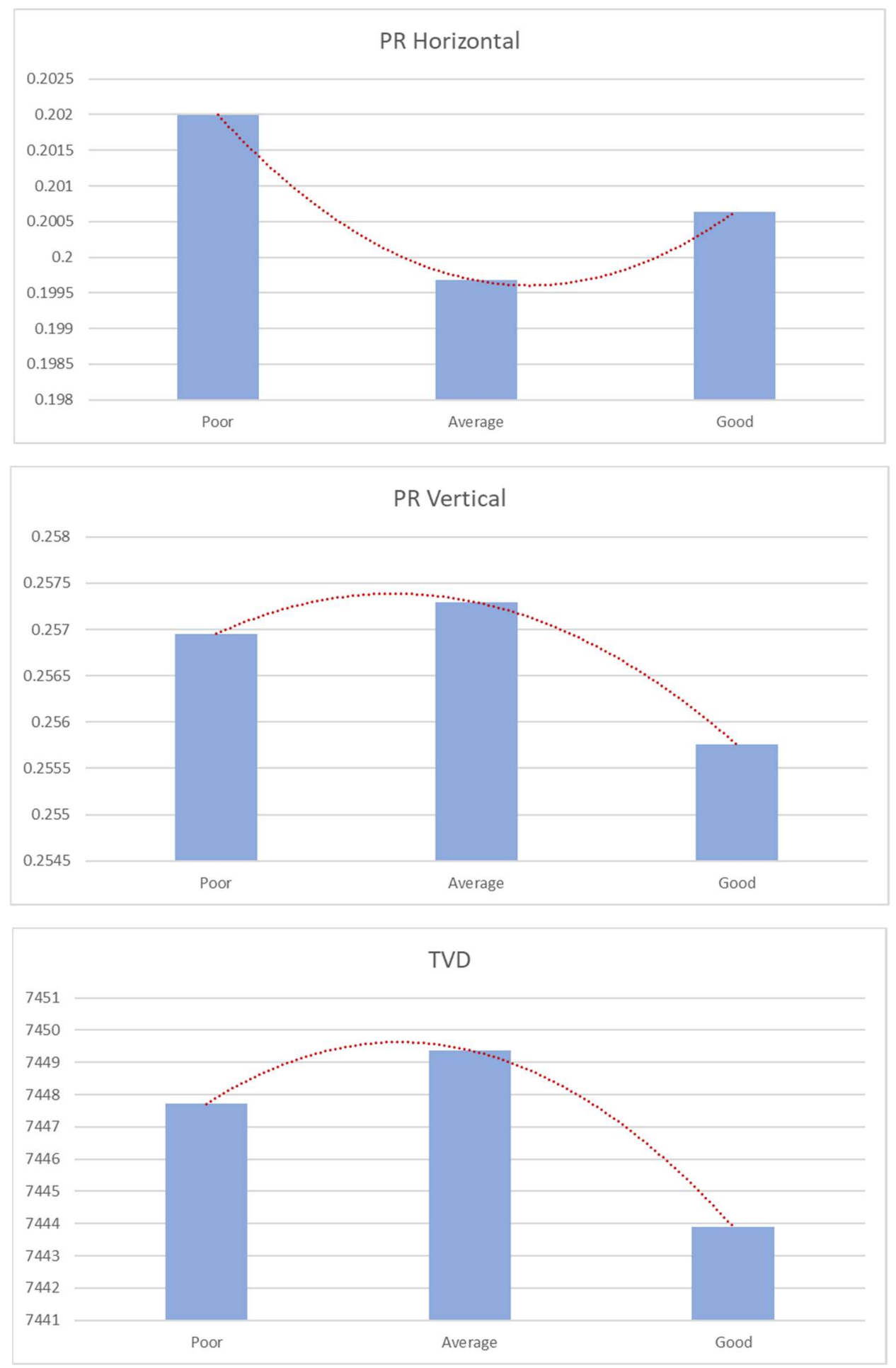

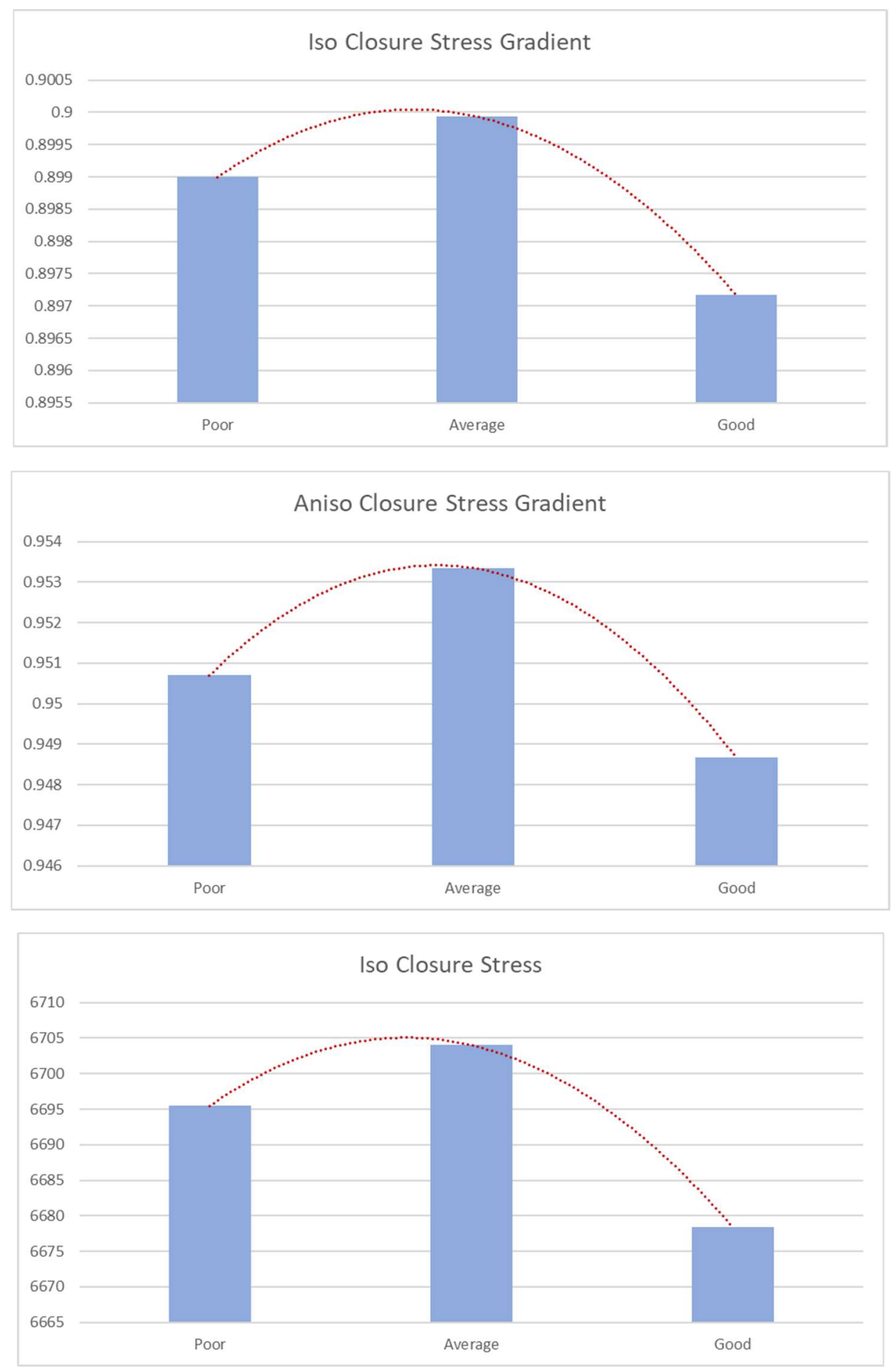

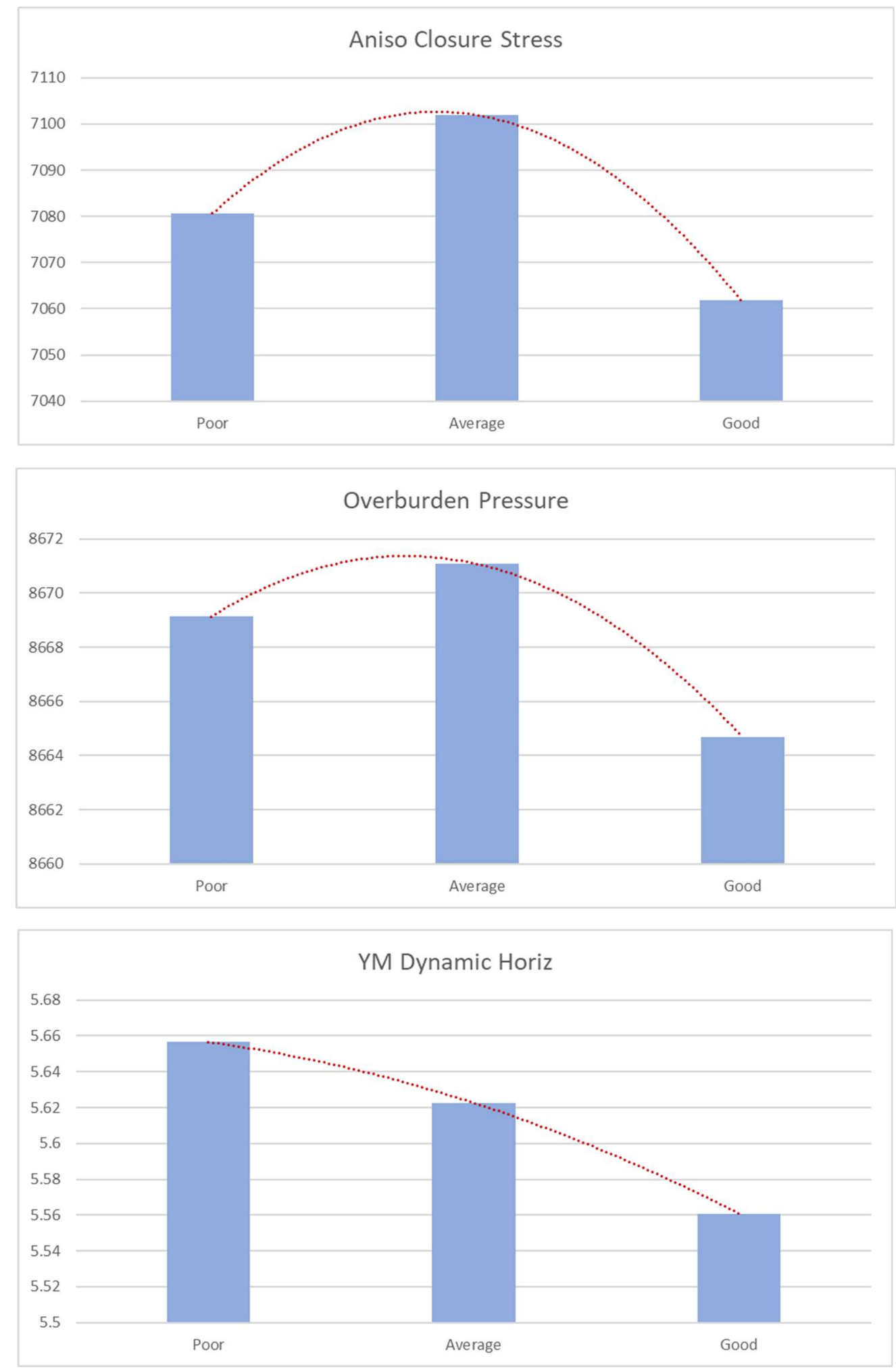

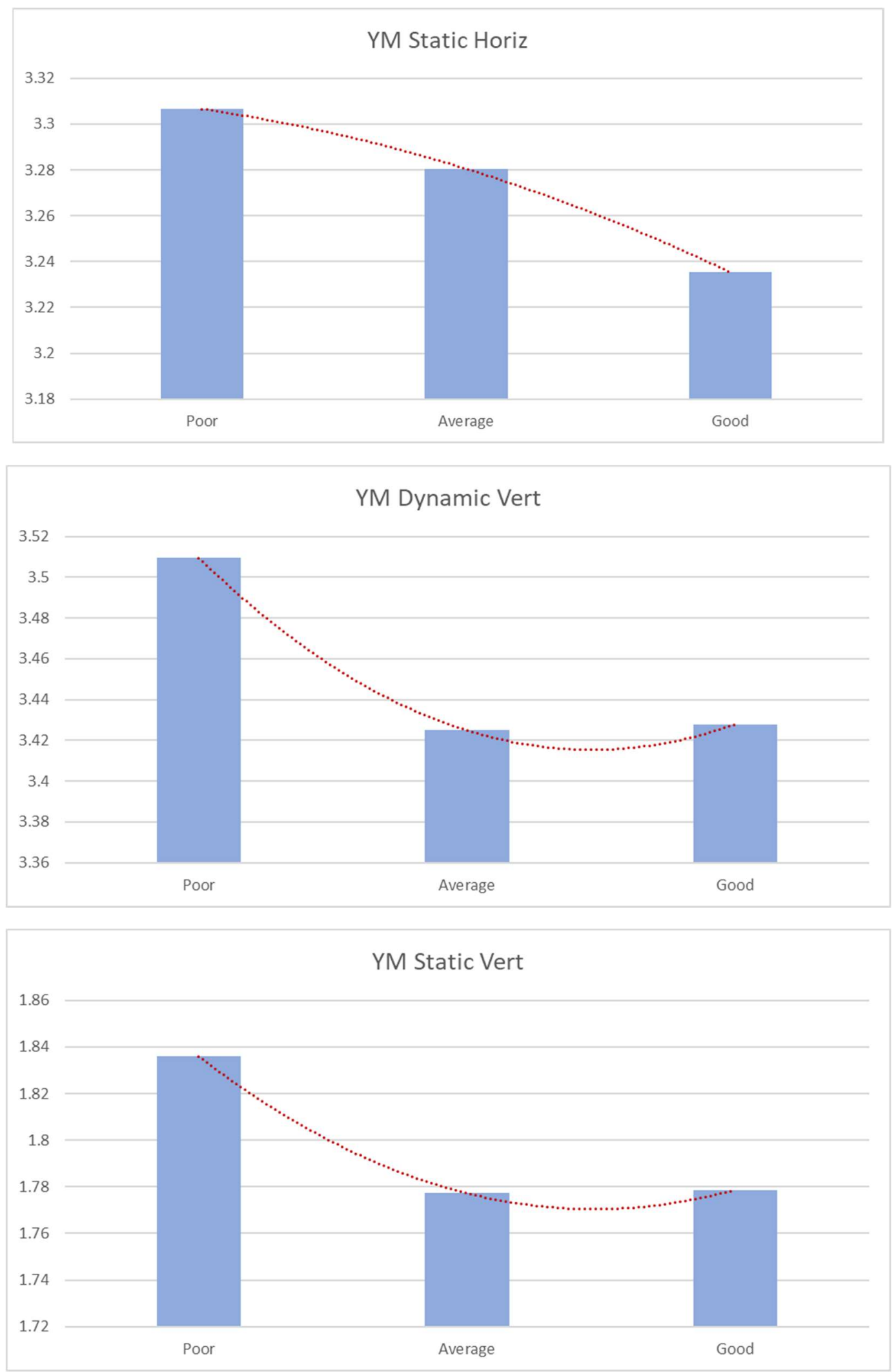
MIP-3H Per-Cluster Classification Plots

Fuzzy Class Assignment vs Production per cluster

Fuzzy Classes:

Class Zero $=$ Blue Bar

Class One $=$ Orange Bar

Class Two $=$ Gray Bar

Production: Purple line

Last cluster of stage:

Red Diamond
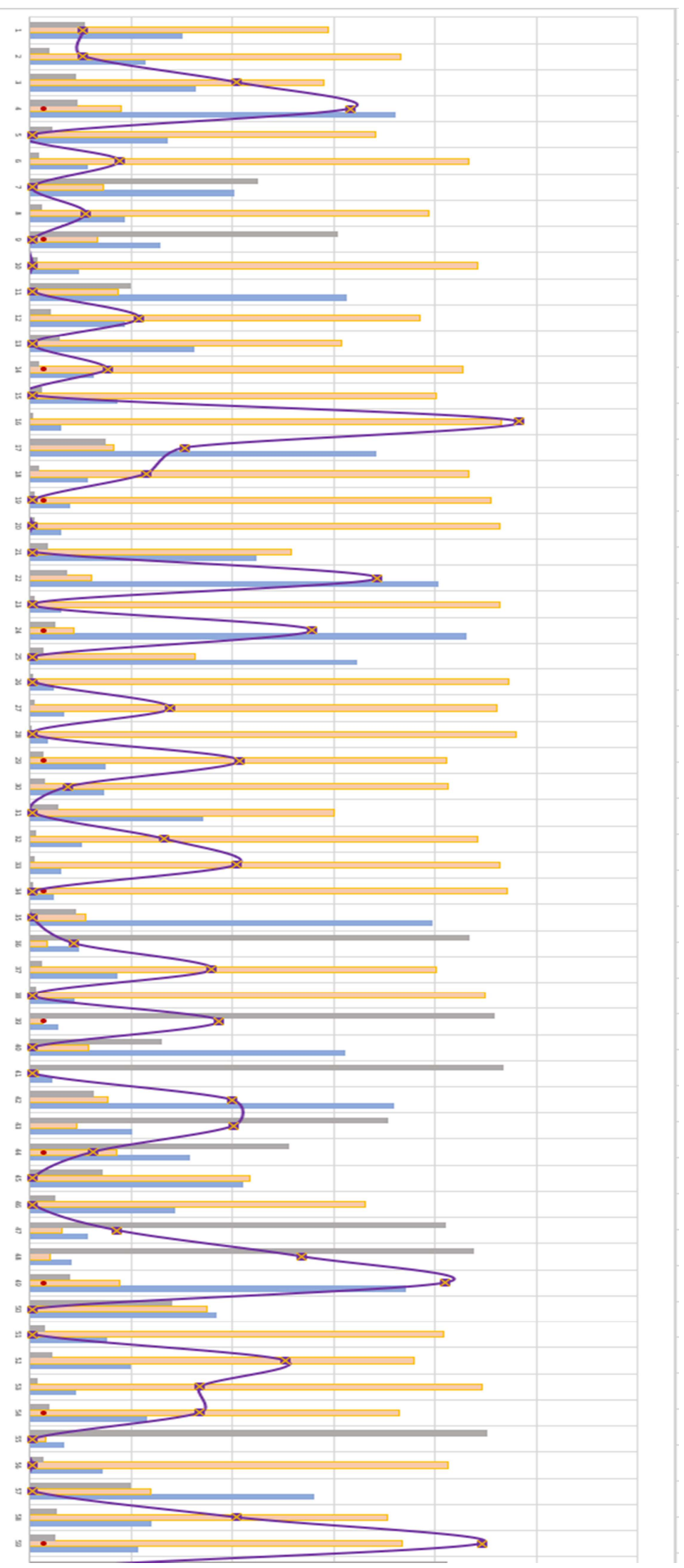


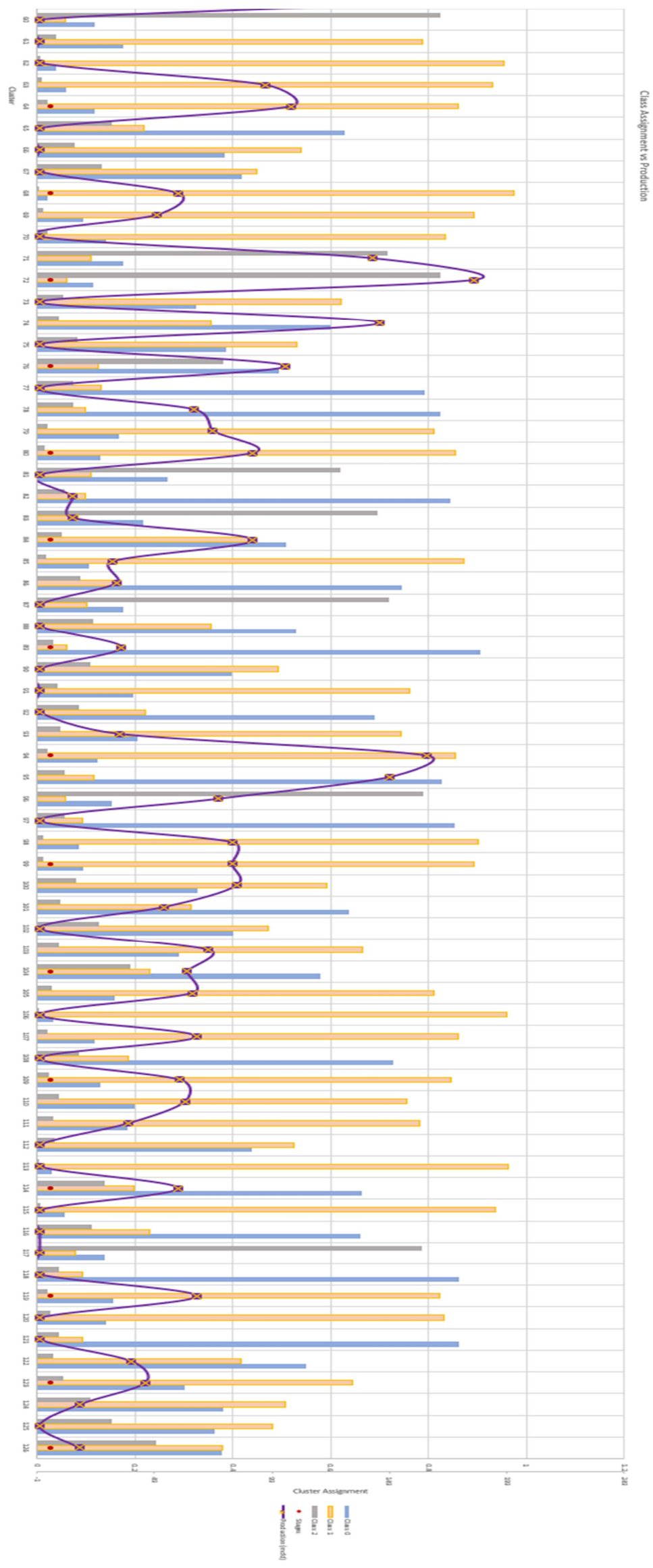




\section{Top Plot - Production vs AIV}

Production: Orange Line

AIV: Blue Line

Last Cluster of Stage: Red

Diamond

Bottom Plot - Production vs

AIV (Inverse)

Production: Orange Line

AIV: Blue Line

Last Cluster of Stage: Red

Diamond
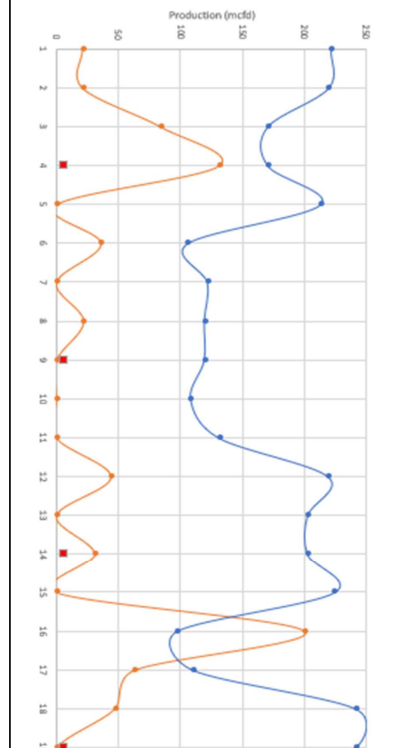

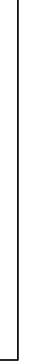
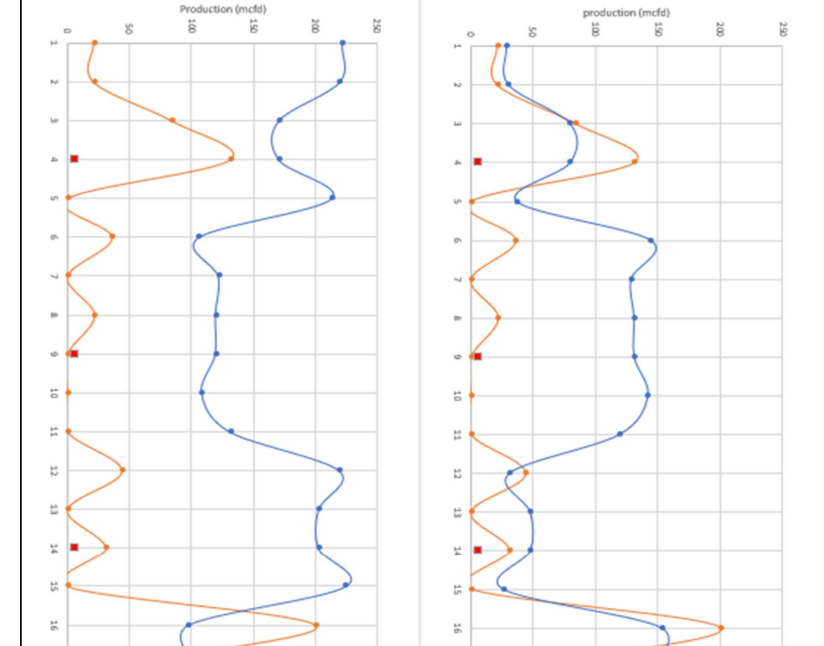
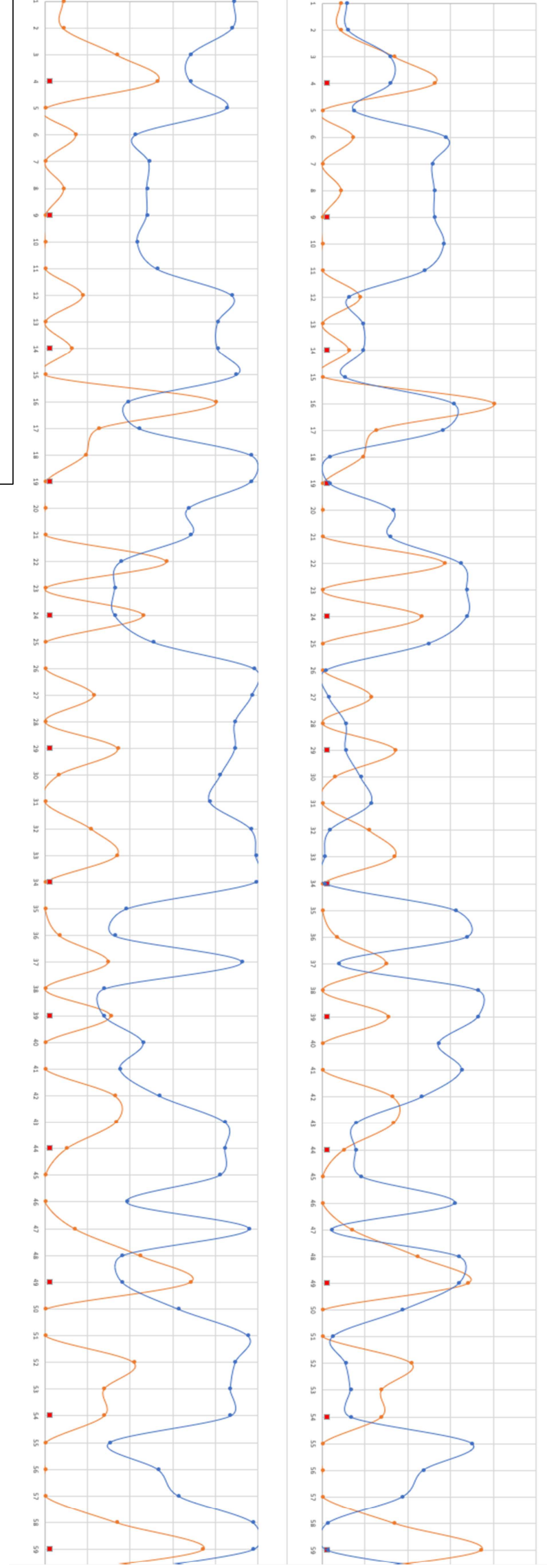

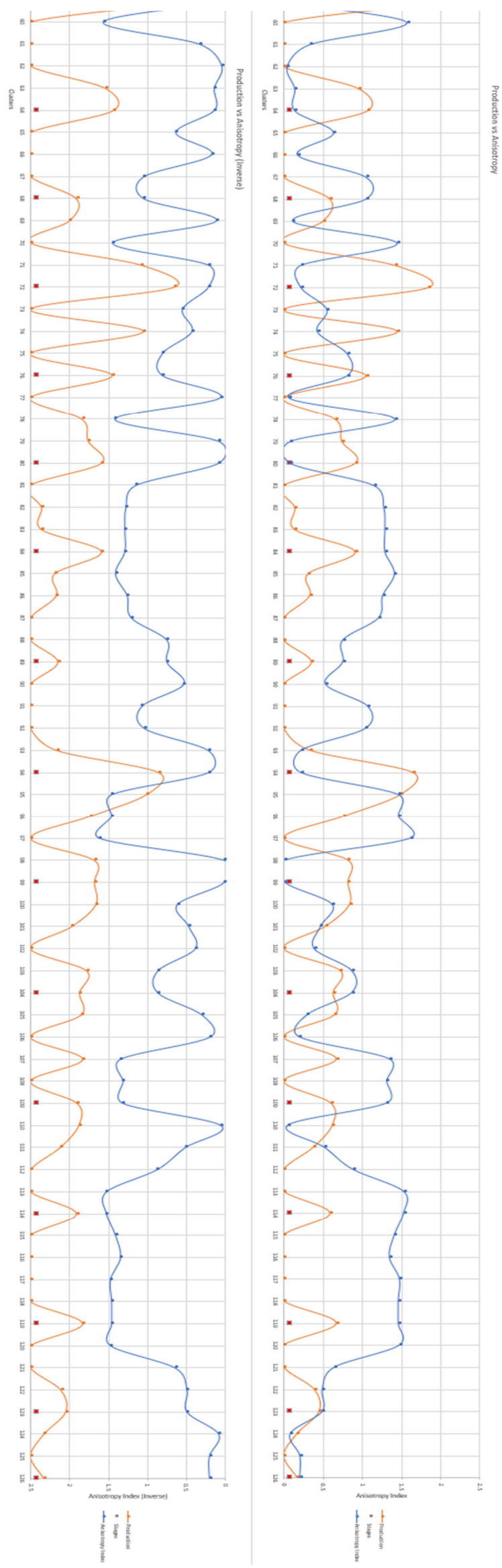


\section{MIP-3H Cluster Re-Design using C-Means}

Stage \#2
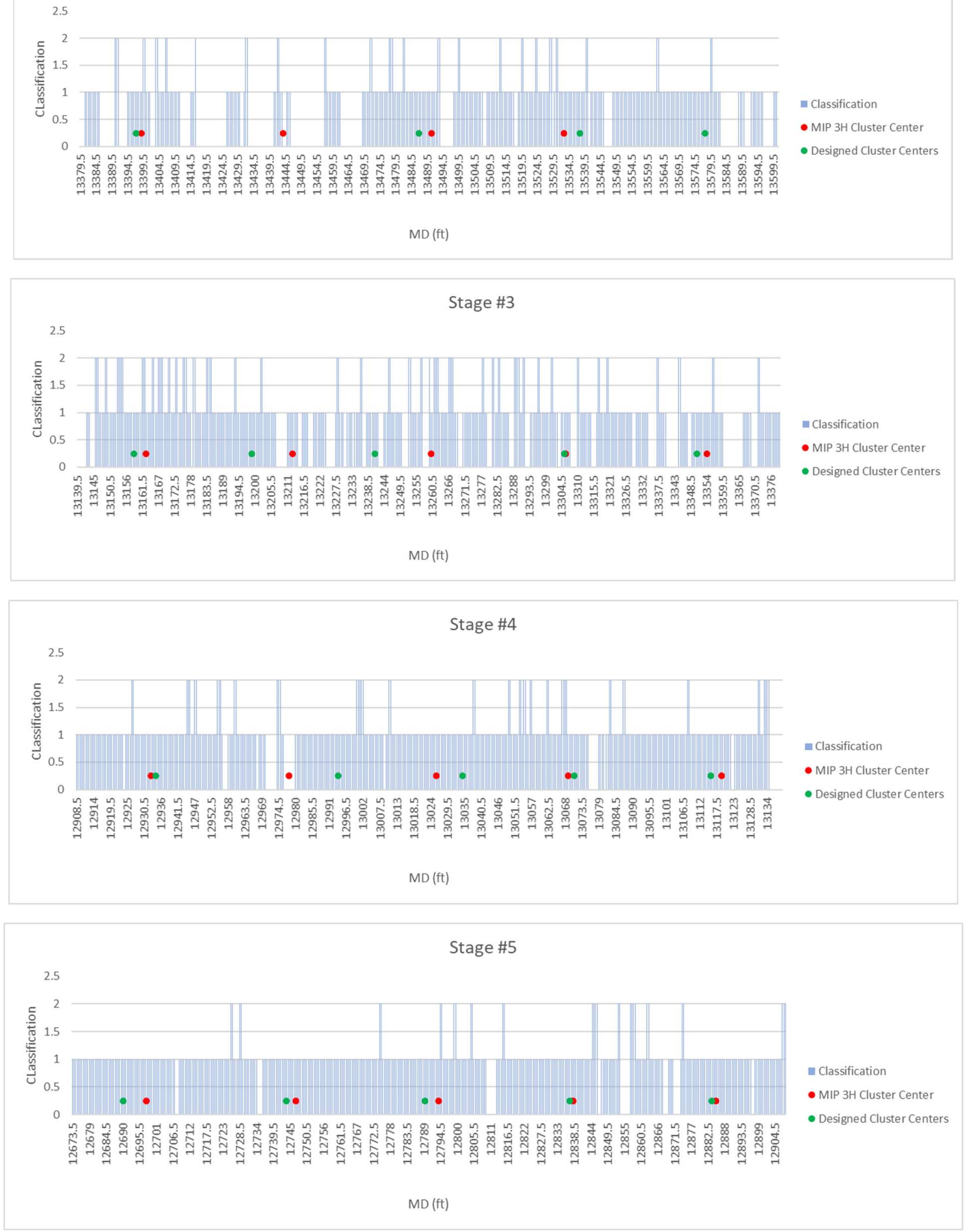

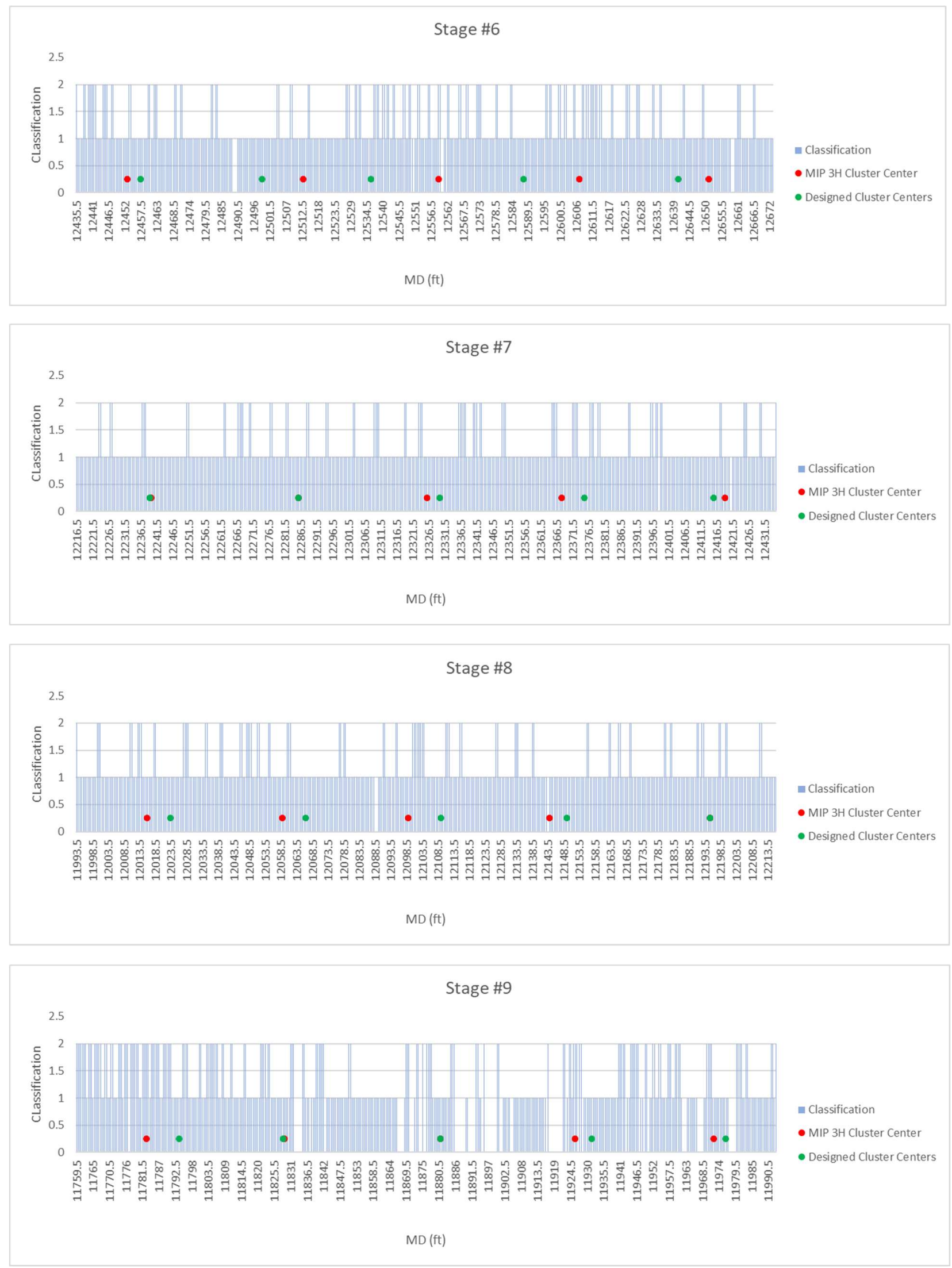

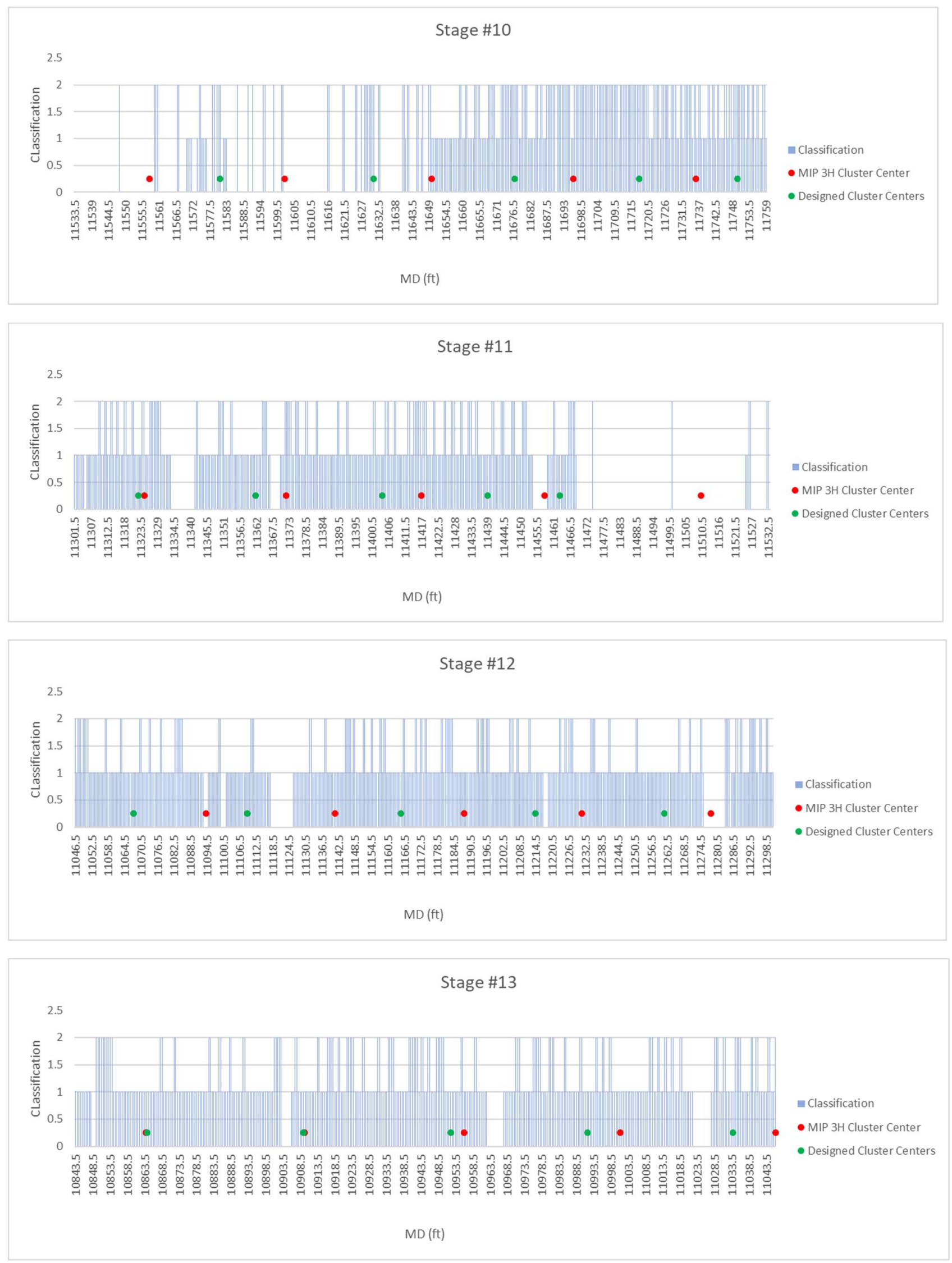

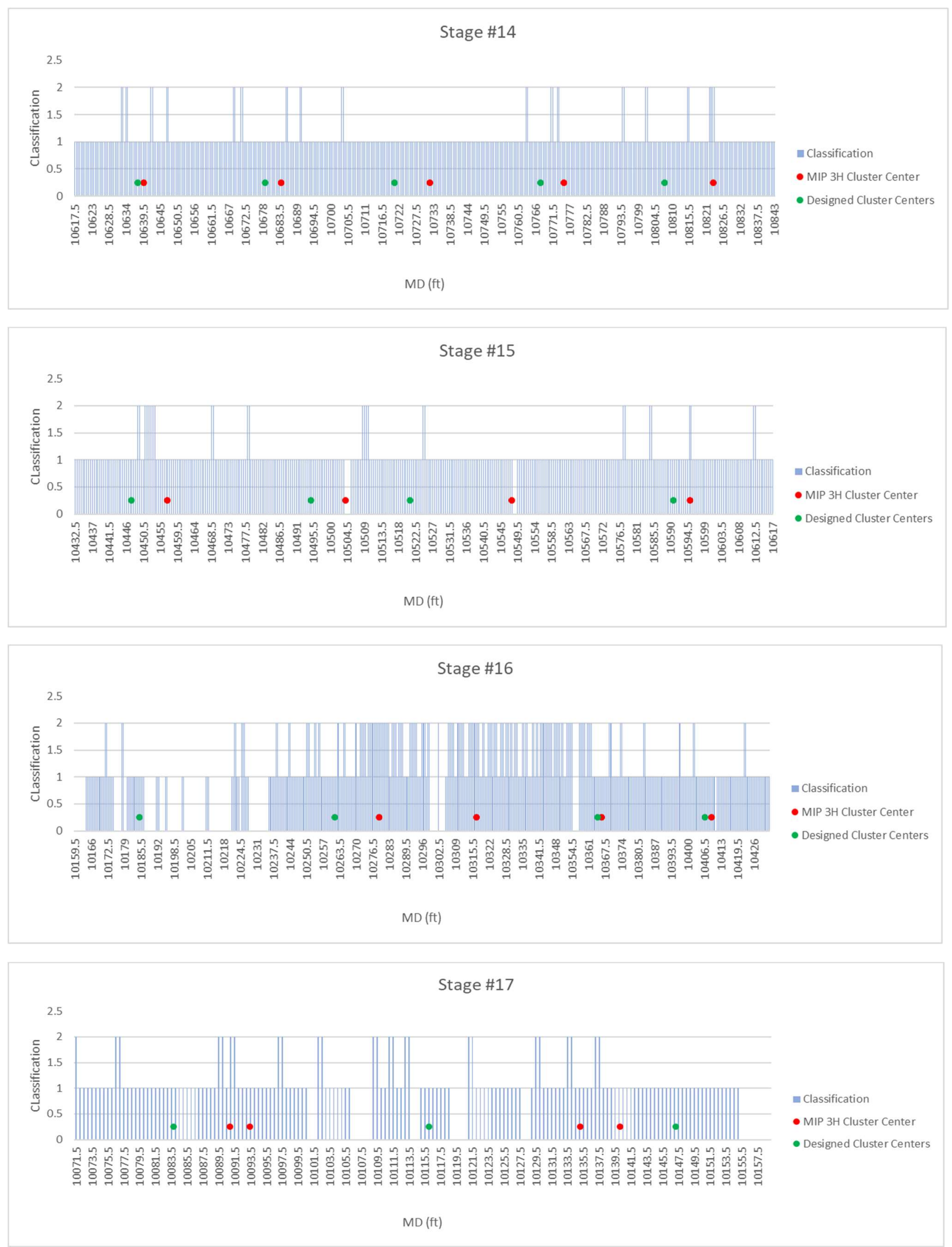

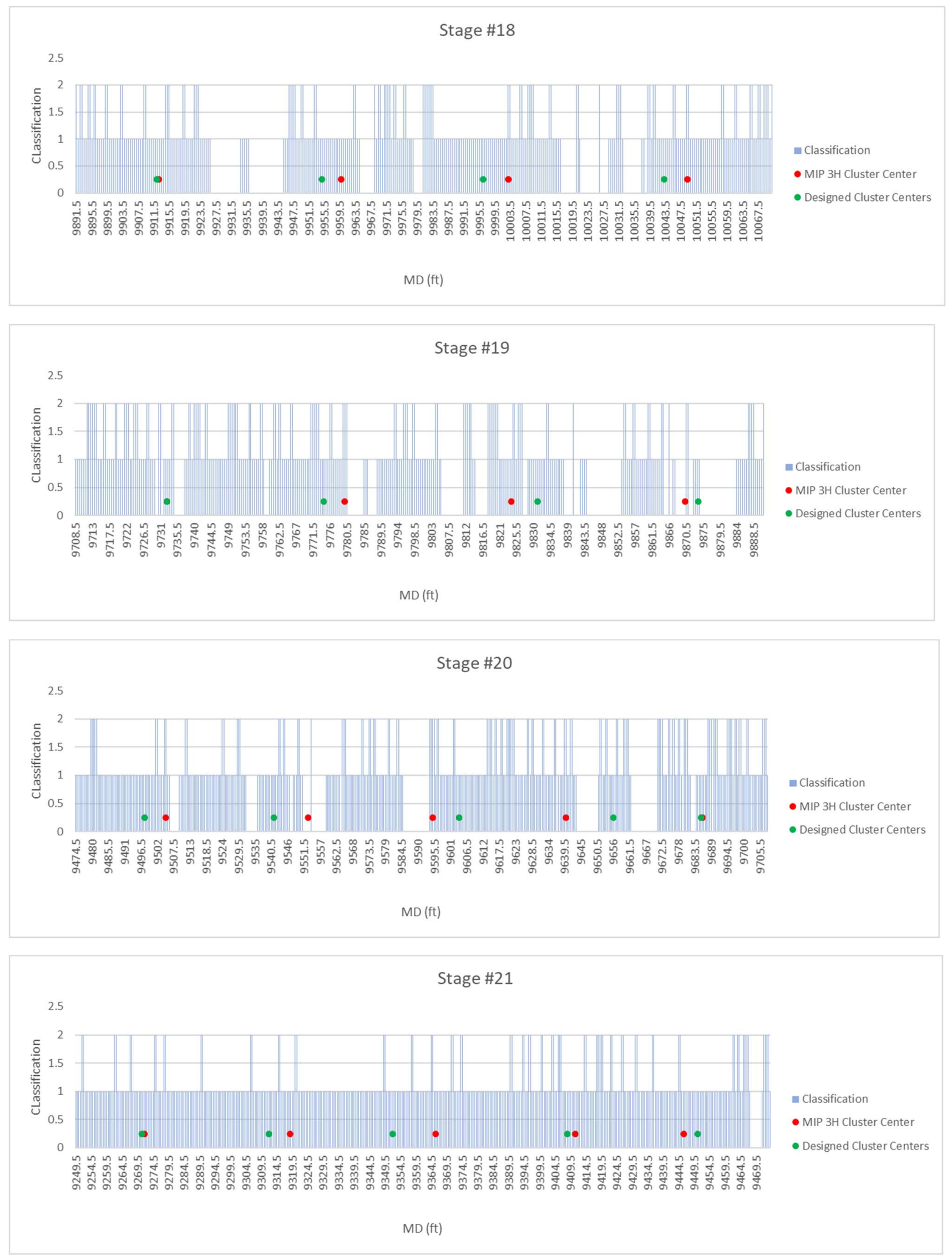

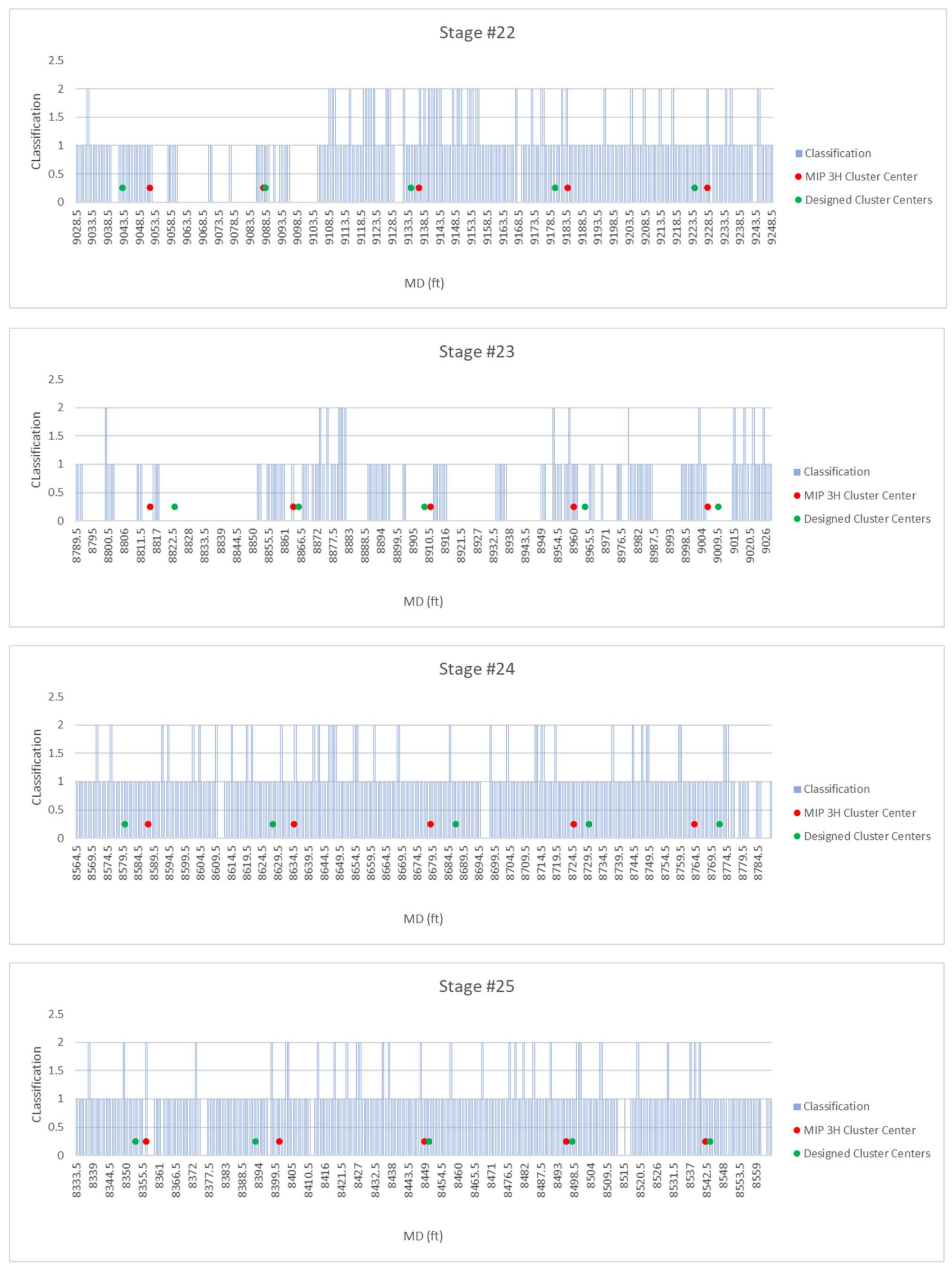

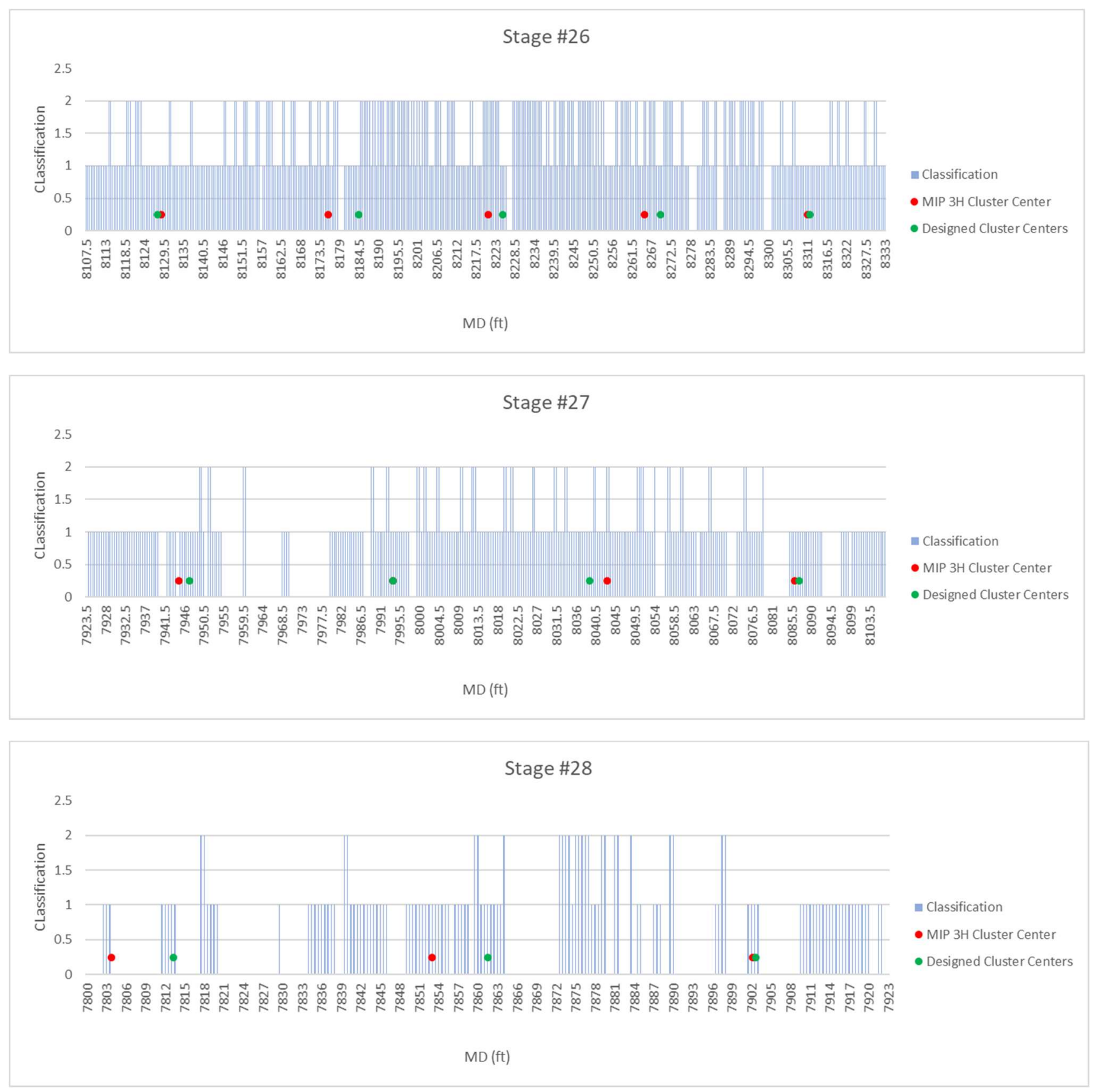


\section{Full MIP-3H Stage Design}

- This plot shows the designed stages (labeled in Red, outlined in yellow/blue alternating) based on the dominant class (blue) and AIV (orange) per half foot.

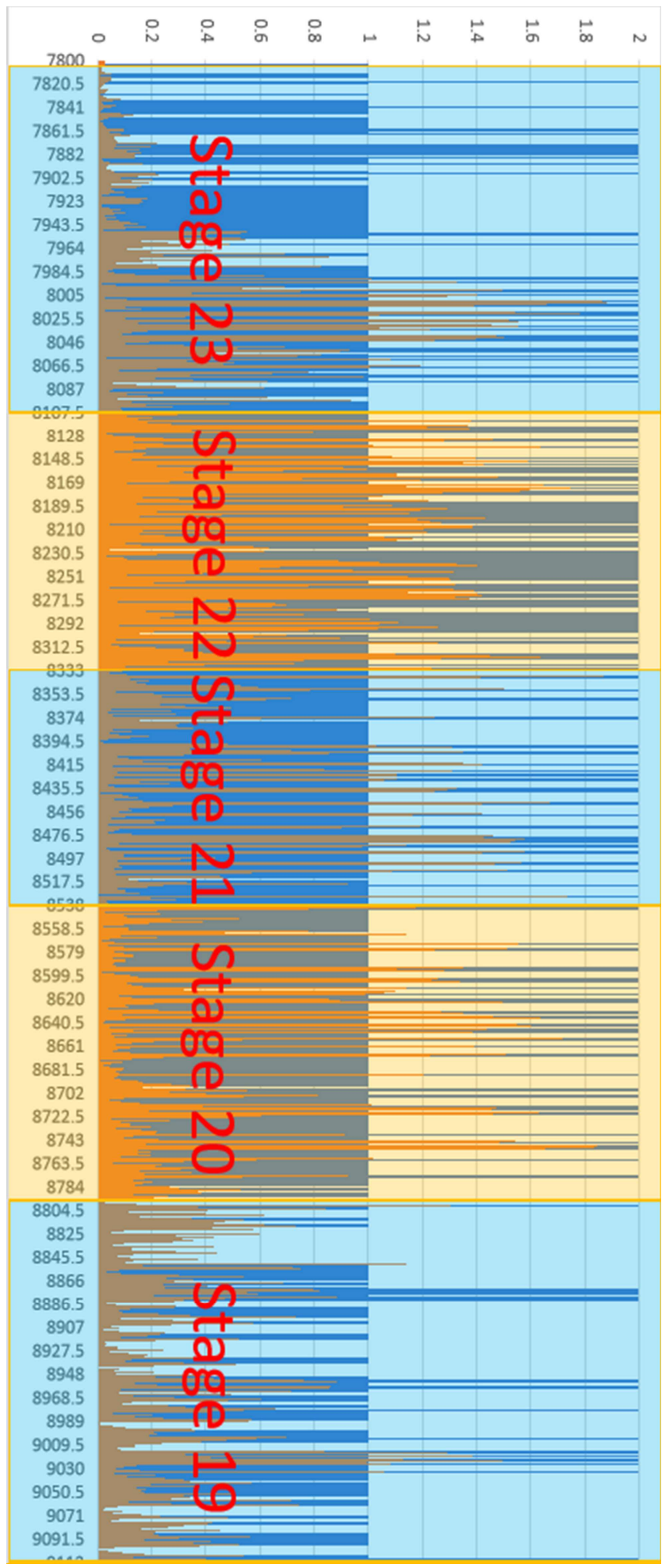




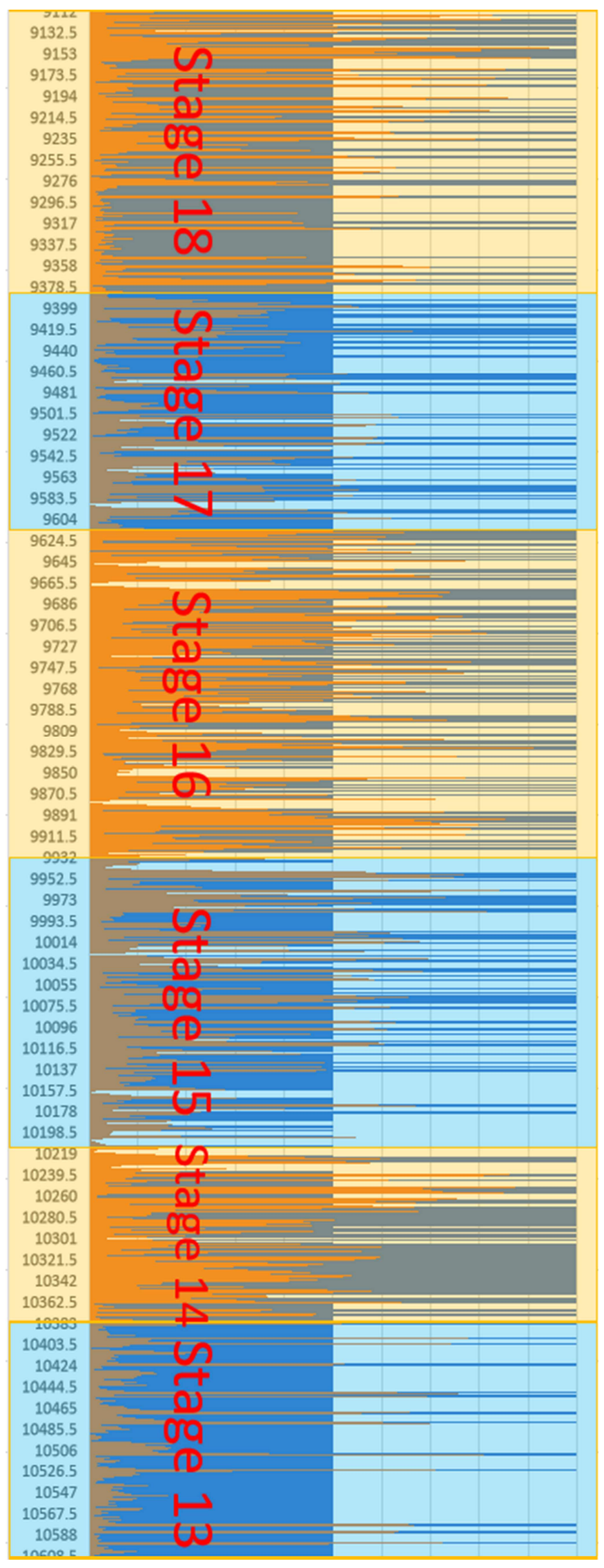




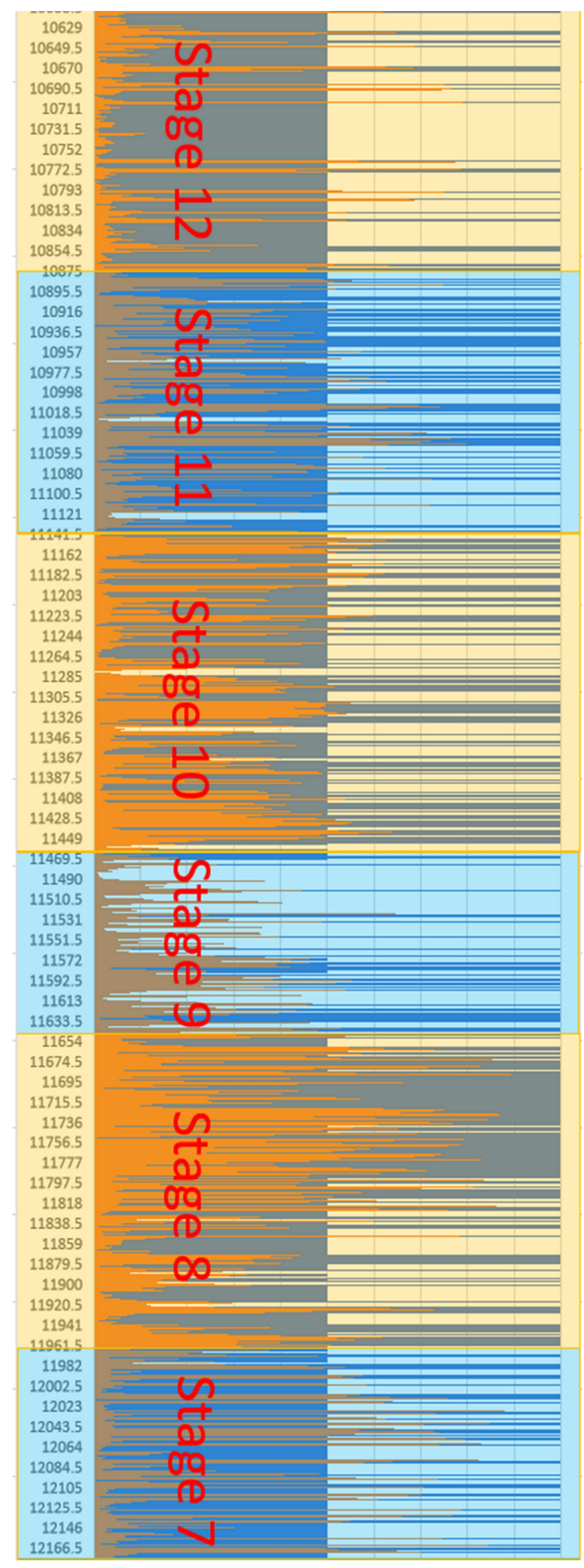




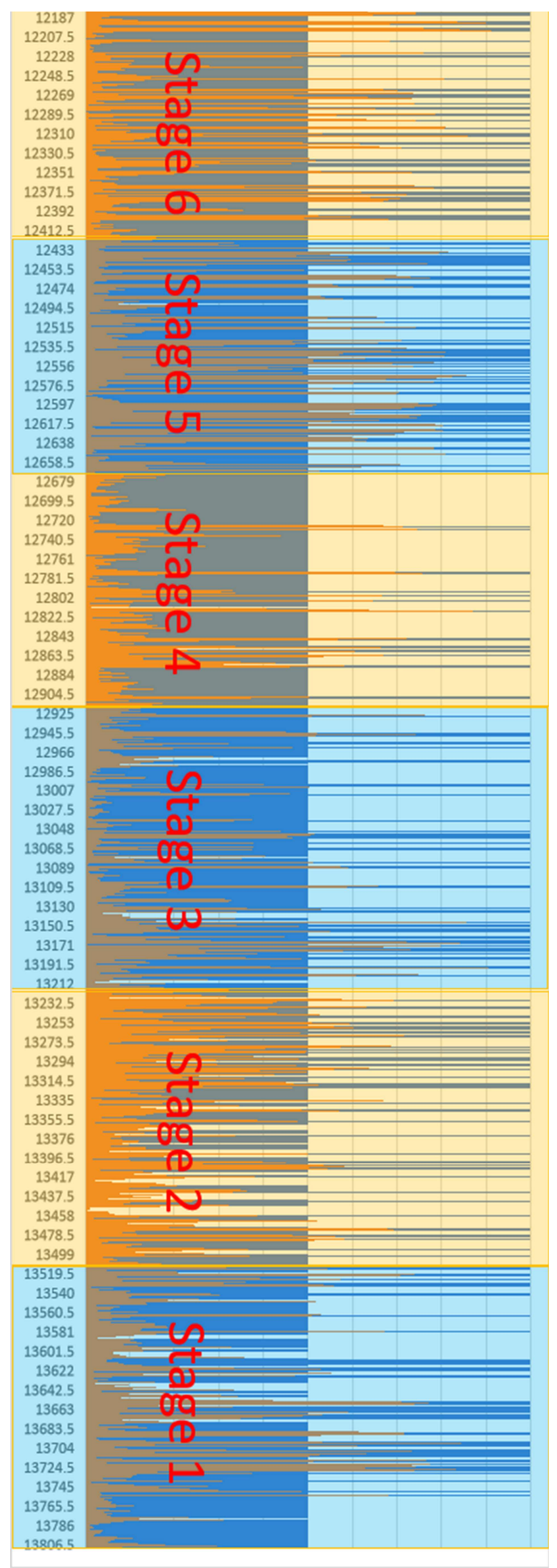




\section{MIP-3H Stage and Cluster Re-Design}
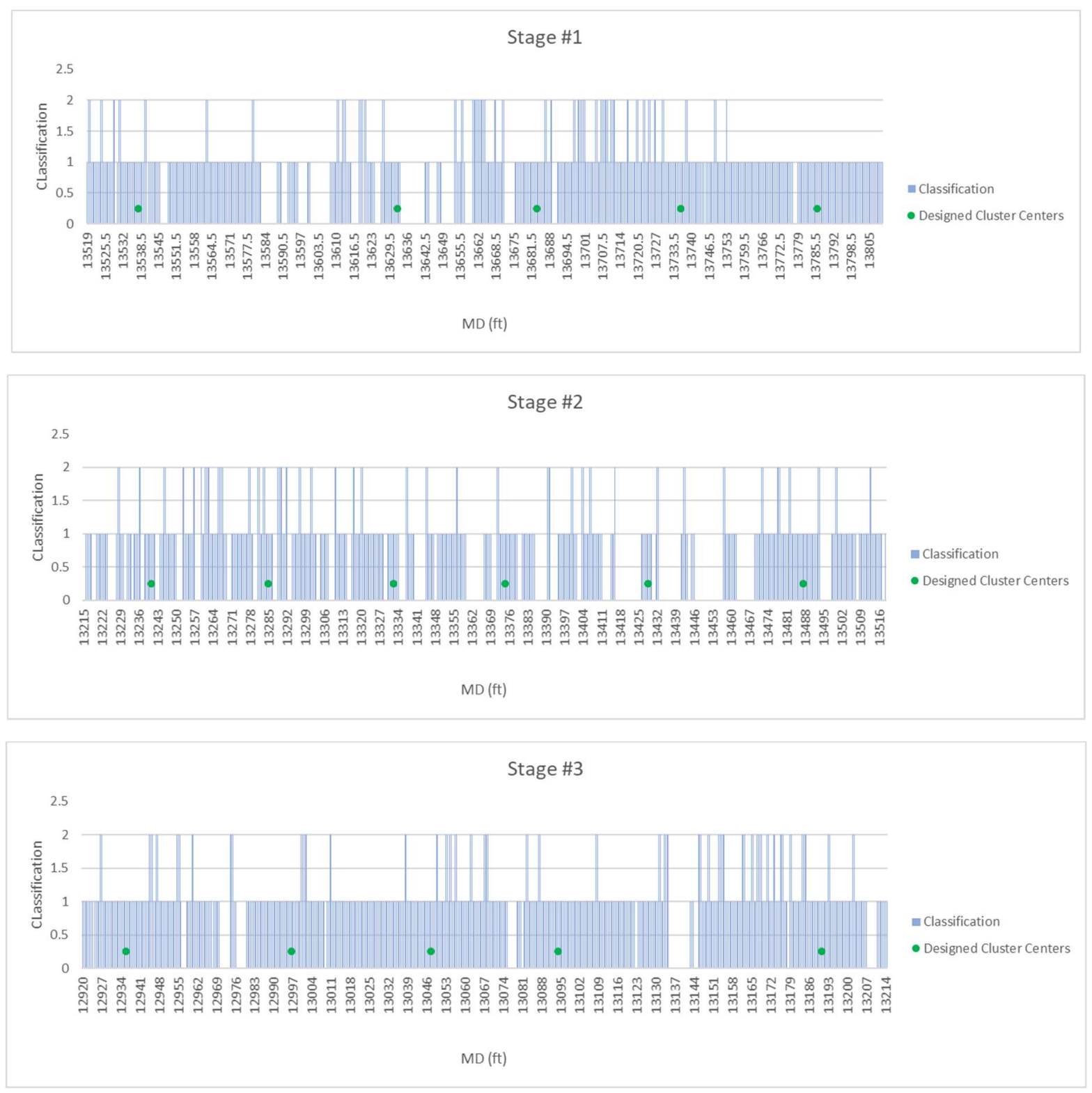

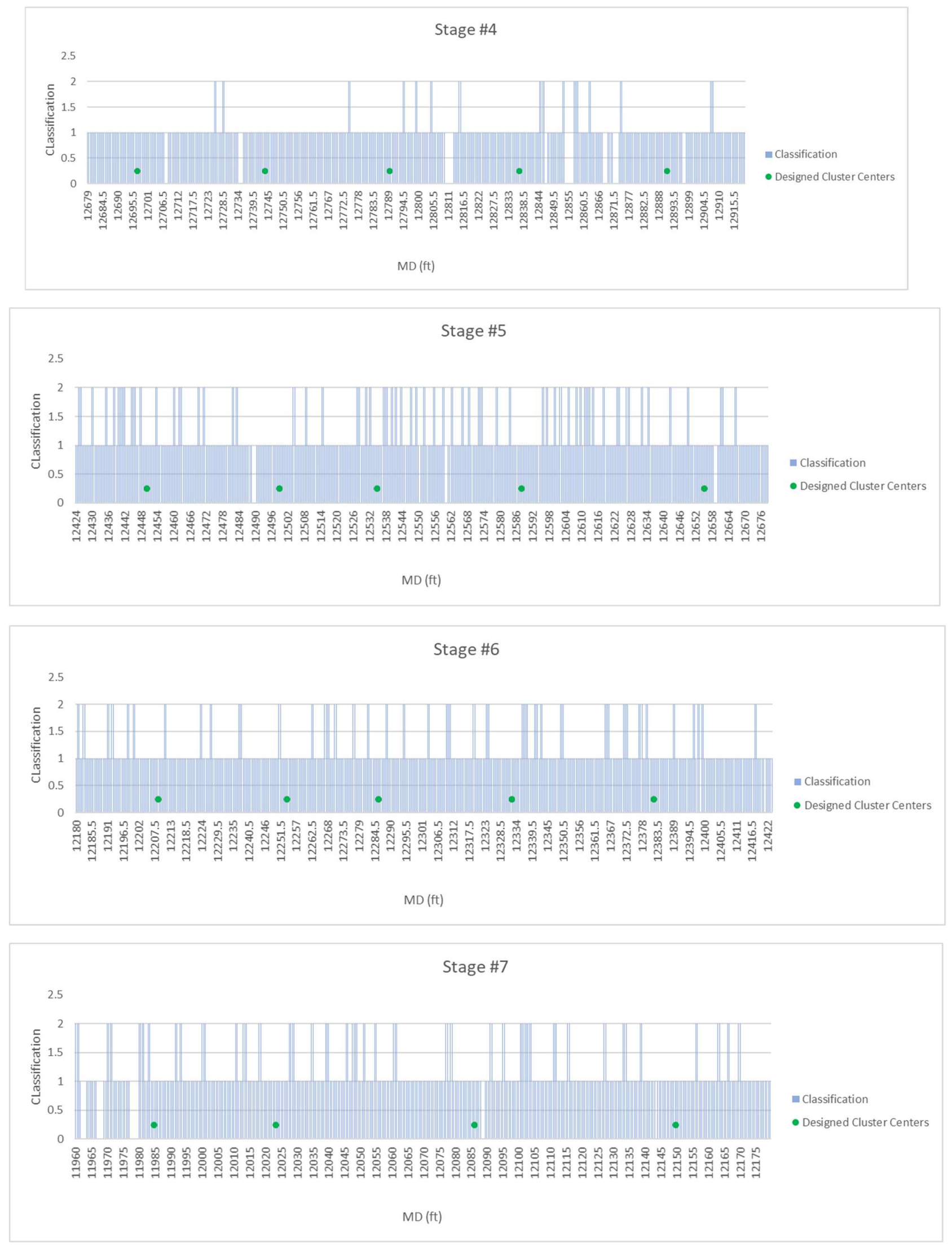

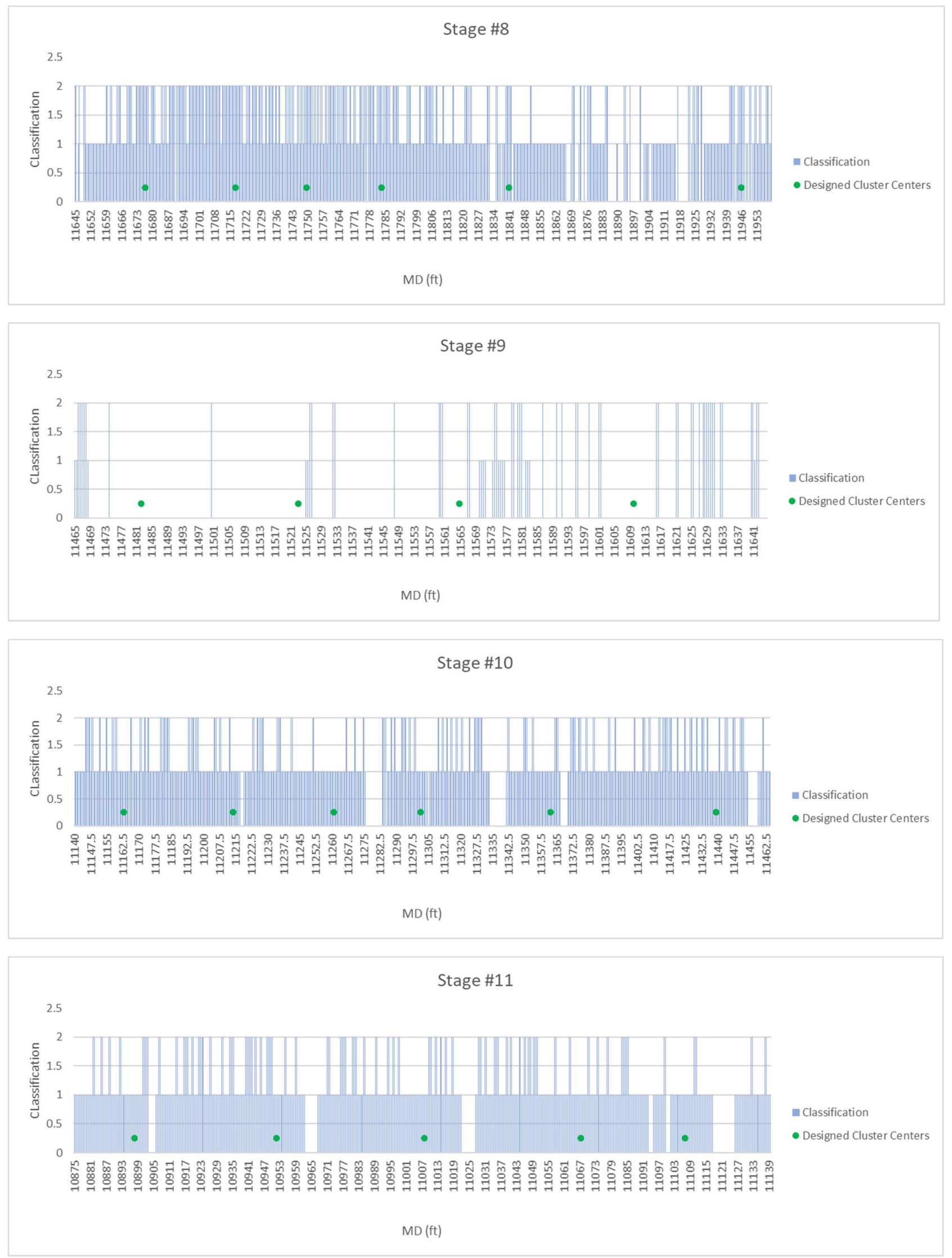

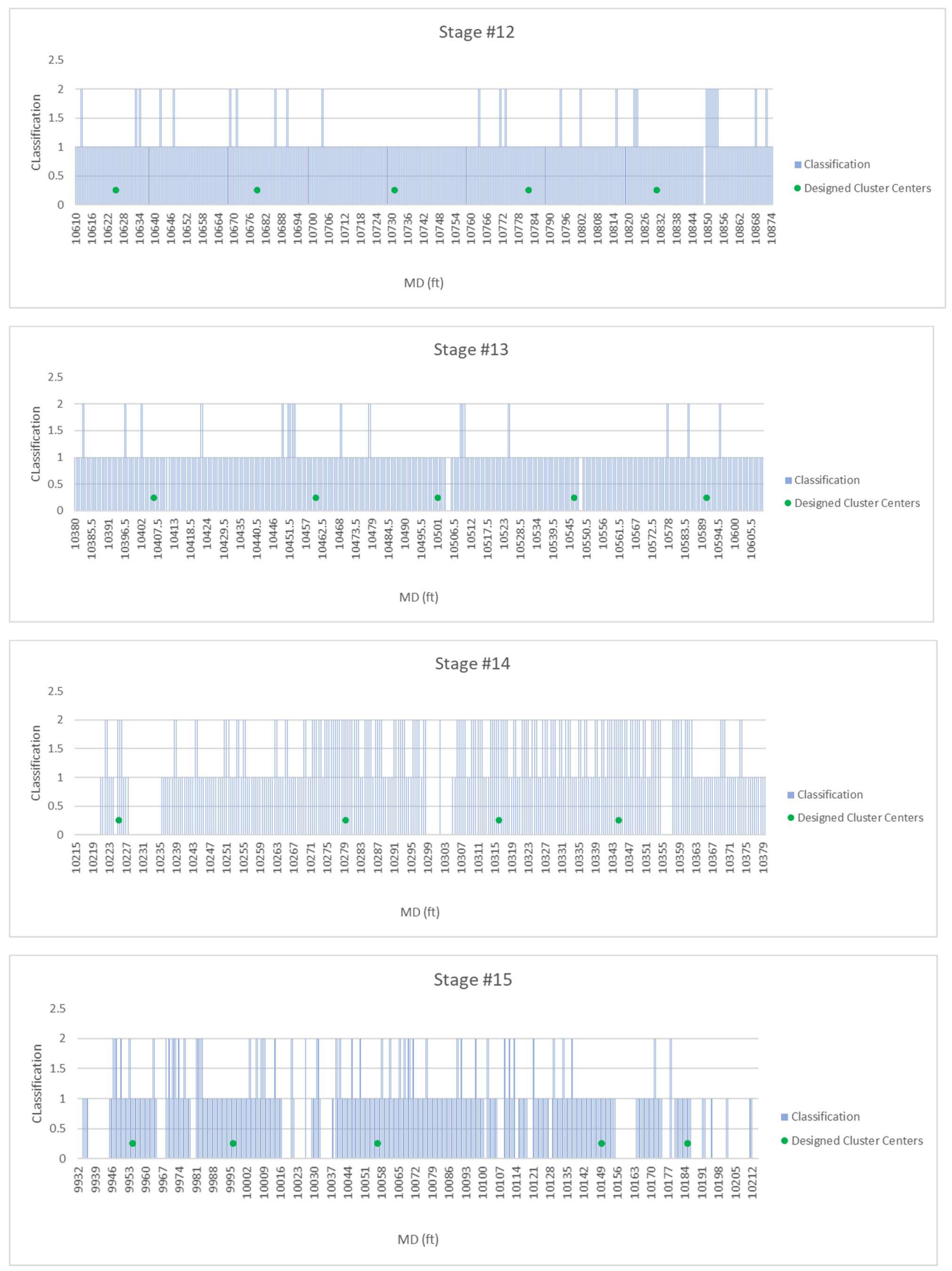

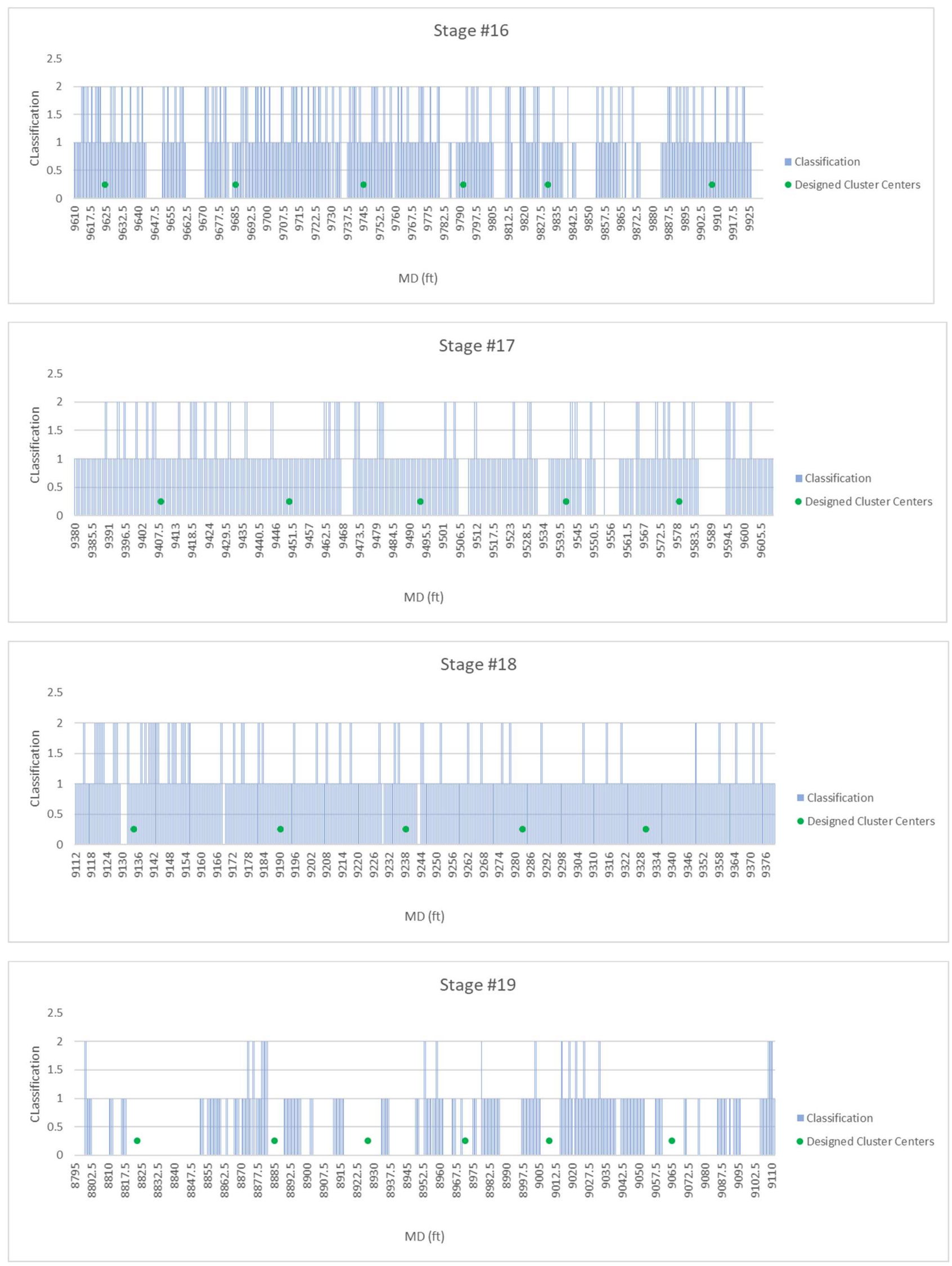

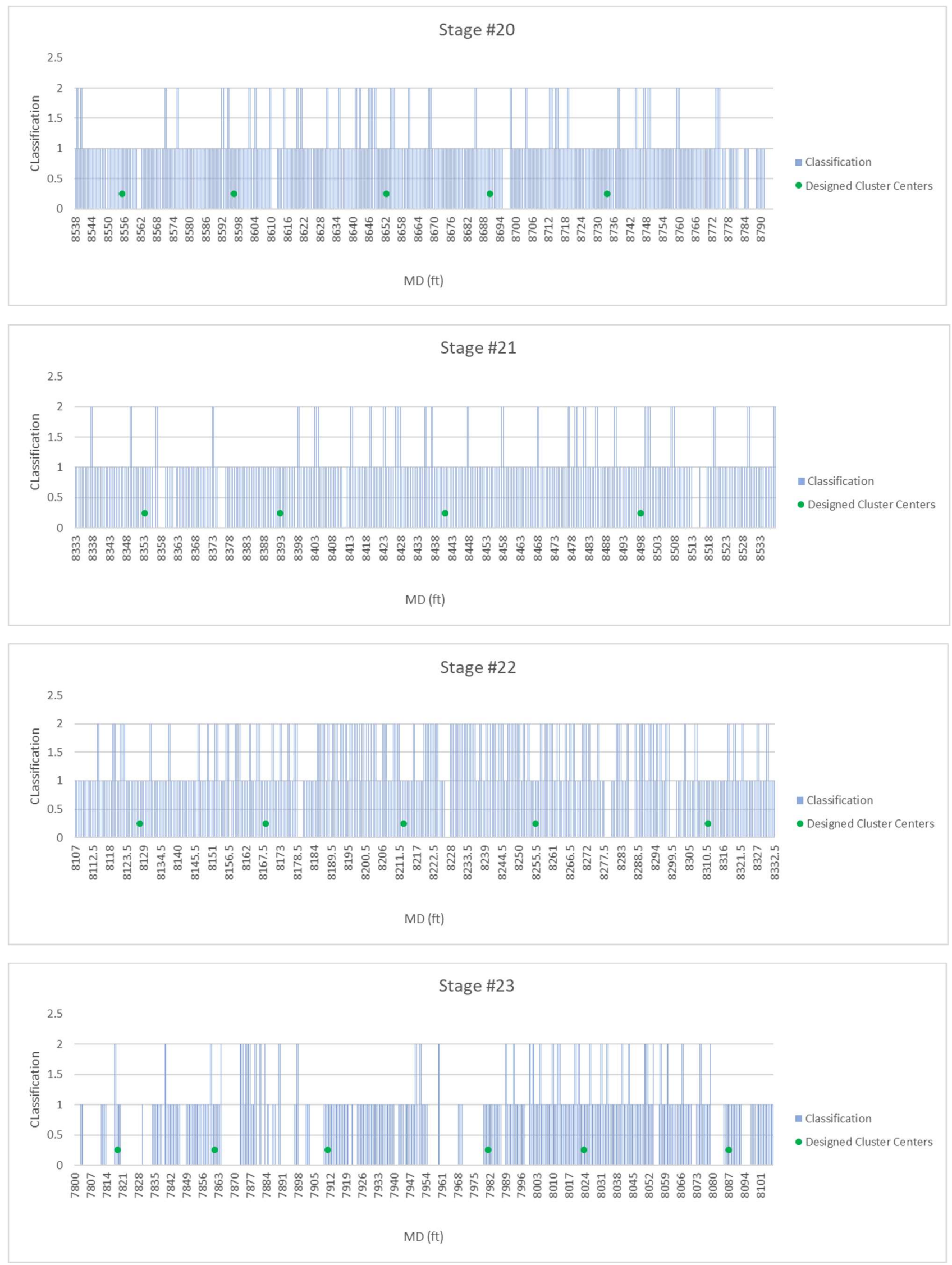